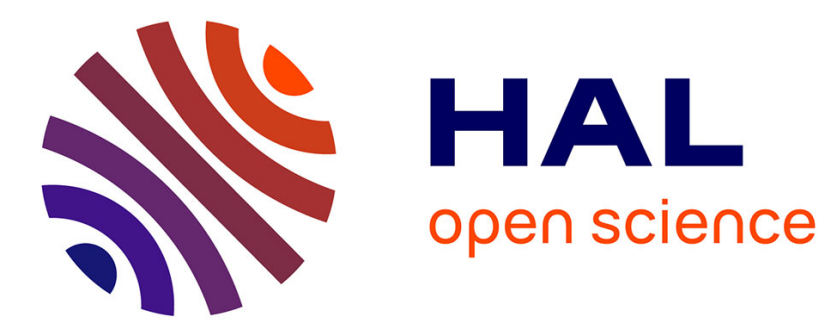

\title{
Convergence of a finite-volume scheme for the Cahn-Hilliard equation with dynamic boundary conditions
}

Flore Nabet

\section{- To cite this version:}

Flore Nabet. Convergence of a finite-volume scheme for the Cahn-Hilliard equation with dynamic boundary conditions. IMA Journal of Numerical Analysis, 2015, 10.1093/imanum/drv057 . hal01096996

\section{HAL Id: hal-01096996 https://hal.science/hal-01096996}

Submitted on 17 Jun 2015

HAL is a multi-disciplinary open access archive for the deposit and dissemination of scientific research documents, whether they are published or not. The documents may come from teaching and research institutions in France or abroad, or from public or private research centers.
L'archive ouverte pluridisciplinaire HAL, est destinée au dépôt et à la diffusion de documents scientifiques de niveau recherche, publiés ou non, émanant des établissements d'enseignement et de recherche français ou étrangers, des laboratoires publics ou privés. 


\title{
CONVERGENCE OF A FINITE-VOLUME SCHEME FOR THE CAHN-HILLIARD EQUATION WITH DYNAMIC BOUNDARY CONDITIONS
}

\author{
F. NABET*
}

\begin{abstract}
This work is devoted to the numerical study of the Cahn-Hilliard equation with dynamic boundary conditions. A spatial finite-volume discretization is proposed which couples a $2 \mathrm{~d}$-method in a smooth connected domain and a 1d-method on its boundary. The convergence of the sequence of approximate solutions is proved and various numerical simulations are given.
\end{abstract} ysis.

Key words. Cahn-Hilliard equation, Dynamic boundary conditions, Finite-volume method, Convergence anal-

AMS subject classifications. 35K55, 65M12, 65M08, 76M12

\section{Introduction.}

1.1. The Cahn-Hilliard equation. The Cahn-Hilliard equation describes the process of phase separation when, for example, a binary allow is cooled down sufficiently. This model is a diffuse interface model because the interface thickness between the two phases is small but non-zero. The system is described by a smooth function $c$ called the order parameter, which is equal to 1 in one of the two phases, 0 in the other and which varies continuously between 0 and 1 in the interfaces.

We consider a connected and bounded domain $\Omega \subset \mathbb{R}^{2}$ with a $\mathcal{C}^{3,1}$-continuous boundary $\Gamma$ and we choose an orientation of $\Gamma$ such that $\overrightarrow{\mathbf{n}}$ is the unit normal vector outward to $\Omega$.

For a given final time $T>0$, the problem is written as follows: To find the concentration of one of the two phases $c:[0, T] \times \Omega \rightarrow \mathbb{R}$ such that,

$$
\left\{\begin{aligned}
\partial_{t} c & =\Gamma_{b} \Delta \mu, \\
\mu & =-\frac{3}{2} \varepsilon \sigma_{b} \Delta c+\frac{12}{\varepsilon} \sigma_{b} f_{b}^{\prime}(c) ;
\end{aligned}\right.
$$

where $\varepsilon>0$ accounts for the interface thickness (see Fig. $1.1 \mathrm{~b}$ ), $f_{b}$ is the bulk Cahn-Hilliard potential, $\Gamma_{b}>0$ is a bulk mobility coefficient and $\sigma_{b}>0$ is a fluid-fluid surface tension coefficient. This is supplemented by an initial condition in $\Omega$,

$$
c(0, .)=c_{0} .
$$

In order to solve this equation, we have to add two boundary conditions on $\Gamma=\partial \Omega$. With respect to the chemical potential $\mu$, we assure that there cannot be any mass exchange through the boundary, thus we consider the homogeneous Neumann boundary condition on $(0, T) \times \Gamma$,

$$
\partial_{n} \mu=0 .
$$

Usually, the boundary condition associated with the order parameter $c$ is the Neumann boundary condition. However, for some physical systems this condition is too restrictive. Indeed, the homogeneous Neumann boundary condition on $c$ implies that the contact angle between the interface and the wall is equal to $\frac{\pi}{2}$. But in some physical systems, for example for binary mixture, the dynamic contact angle deviate from the static contact angle $\frac{\pi}{2}$. In order to

* flore.nabet@univ-amu.fr. Aix Marseille Université, CNRS, Centrale Marseille, I2M, UMR 7373, 13453 Marseille, France 
describe this phenomenon, physicists [12, 13, 16] have introduced the following boundary condition on $(0, T) \times \Gamma$, called the dynamic boundary condition,

$$
\frac{1}{64} \frac{\varepsilon^{3}}{\Gamma_{b} \Gamma_{s}} \partial_{t} c_{\text {IГ }}=\frac{3}{8} \varepsilon^{2} \sigma_{b} \sigma_{s} \Delta_{\Gamma} c_{\mid \Gamma}-6 \sigma_{b} f_{s}^{\prime}\left(c_{1 \Gamma}\right)-\frac{3}{2} \varepsilon \sigma_{b} \partial_{n} c .
$$

The trace of $c$ on $\Gamma$ is noted $c_{1 \Gamma}, \Delta_{\Gamma}$ is the Laplace-Beltrami operator on $\Gamma, \partial_{n}$ is the normal derivative at the boundary, $\Gamma_{s}>0$ is a surface kinetic coefficient and $\sigma_{s}>0$ is a surface capillarity coefficient. The bulk potential $f_{b}$ and the surface potential $f_{s}$ satisfy the following assumptions.

- Dissipativity

$$
\liminf _{|c| \rightarrow \infty} f_{b}^{\prime \prime}(c)>0 \quad \text { and } \quad \liminf _{|c| \rightarrow \infty} f_{s}^{\prime \prime}(c)>0 .
$$

These conditions imply that there exist $\alpha_{1}>0$ and $\alpha_{2} \geq 0$ such that for all $x \in \mathbb{R}$,

$$
f_{b}(c) \geq \alpha_{1} c^{2}-\alpha_{2} \quad \text { and } \quad f_{s}(c) \geq \alpha_{1} c^{2}-\alpha_{2} .
$$

- Polynomial growth for $f_{b}$ : there exist $C_{b}>0$ and a real $p \geq 2$ such that,

$$
\left|f_{b}^{(m)}(c)\right| \leq C_{b}\left(1+|c|^{p-m}\right), \quad m=\{0,1,2\} .
$$

A typical choice for the bulk potential is the polynomial double-well function (see Fig. 1.1a).

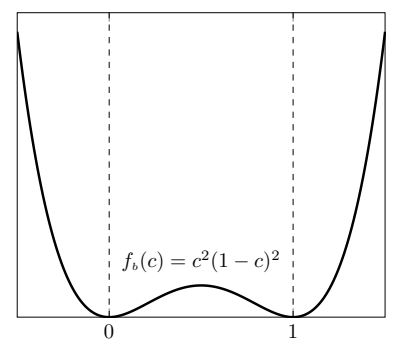

(a) Bulk potential

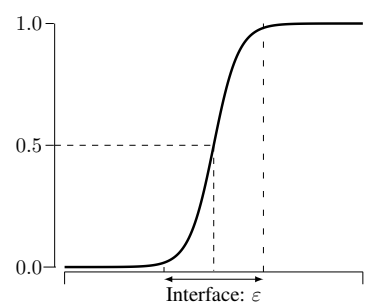

(b) Interface thickness

Fig. 1.1: Double-well structure of $f_{b}$ and definition of the interface thickness

For the sake of simplicity, we note

$$
\mathcal{A}_{\Delta}=\frac{3}{2} \varepsilon \sigma_{b}, \quad \mathcal{A}_{f_{b}}=\frac{12}{\varepsilon} \sigma_{b}, \mathcal{A}_{\partial t}=\frac{1}{64} \frac{\varepsilon^{3}}{\Gamma_{b} \Gamma_{s}}, \mathcal{A}_{\Delta_{\Gamma}}=\frac{3}{8} \varepsilon^{2} \sigma_{b} \sigma_{s} \text { and } \mathcal{A}_{f_{s}}=6 \sigma_{b},
$$

and we write the Cahn-Hilliard equation with dynamic boundary conditions as follows

$$
\left\{\begin{aligned}
\partial_{t} c & =\Gamma_{b} \Delta \mu ; & & \text { in }(0, T) \times \Omega ; \\
\mu & =-\mathcal{A}_{\Delta} \Delta c+\mathcal{A}_{f_{b}} f_{b}^{\prime}(c) ; & & \text { in }(0, T) \times \Omega ; \\
\mathcal{A}_{\partial t} \partial_{t} c_{\mid \Gamma} & =\mathcal{A}_{\Delta_{\Gamma}} \Delta_{\Gamma} c_{1 \Gamma}-\mathcal{A}_{f_{s}} f_{s}^{\prime}\left(c_{1 \Gamma}\right)-\mathcal{A}_{\Delta} \partial_{n} c ; & & \text { on }(0, T) \times \Gamma ; \\
\partial_{n} \mu & =0 ; & & \text { on }(0, T) \times \Gamma ; \\
c(0, .) & =c^{0} ; & & \text { in } \Omega .
\end{aligned}\right.
$$


The free energy functional associated with this equation is decomposed into a bulk and a surface contribution

$$
\mathcal{F}(c)=\mathcal{F}_{b}(c)+\mathcal{F}_{s}(c)
$$

where the bulk contribution is the usual energy functional associated with the Cahn-Hilliard equation with Neumann boundary conditions

$$
\mathcal{F}_{b}(c)=\int_{\Omega}\left(\frac{\mathcal{A}_{\Delta}}{2}|\nabla c|^{2}+\mathcal{A}_{f_{b}} f_{b}(c)\right) .
$$

As regards the surface contribution, we have

$$
\mathcal{F}_{s}(c)=\int_{\Gamma}\left(\frac{\mathcal{A}_{\Delta_{\Gamma}}}{2}\left|\nabla_{\Gamma} c_{\mid \Gamma}\right|^{2}+\mathcal{A}_{f_{s}} f_{s}\left(c_{1 \Gamma}\right)\right)
$$

and we can note that the dynamic boundary condition $(1.4)$ is obtained by requiring that the system tends to minimize its total free energy $\mathcal{F}$. Indeed, with this definition, the free energy functional is dissipated as follows,

$$
\frac{\mathrm{d}}{\mathrm{d} t} \mathcal{F}(c(t, .))=-\Gamma_{b} \int_{\Omega}|\nabla \mu(t, .)|^{2}-\mathcal{A}_{\partial t} \int_{\Gamma}\left|\partial_{t} c_{1 \Gamma}(t, .)\right|^{2}, \quad t \in[0, T[.
$$

We can see that the natural energy space for this problem is the following function space,

$$
H_{\Gamma}^{1}(\Omega)=\left\{u \in H^{1}(\Omega): \operatorname{Tr} u=u_{1 \Gamma} \in H^{1}(\Gamma)\right\} .
$$

From a mathematical point of view, the Cahn-Hilliard equation with dynamic boundary conditions is now well understood. We refer the reader to [7, 17, 18, 19, 20] and the references therein for details on the existence, uniqueness and regularity of solutions, existence of attractors and convergence to stationary states. Moreover, in [15] the authors prove (in a more general framework) the existence of solutions to continuous Problem (1.7) in the energy spaces. From a numerical point of view, there are less results. In [12, 13, 16] the authors consider finite-difference methods and give numerical results without proof of convergence. A spatial finite-element semi-discretization is proposed in [6] where the authors prove convergence results and optimal error estimates for the space semi-discrete scheme. All these results are obtained in a slab by imposing periodic conditions in lateral directions. Thus, more complex geometries of the domain are not considered.

In this article, we investigate a finite-volume scheme for solving Problem 1.7) with a smooth domain $\Omega$. This spatial discretization allows to easily couple the dynamics in the domain and those on the boundary by the flux term $\partial_{n} c$. Furthermore, finite-volume schemes account naturally for the non-flat geometry of the boundary and for the associated LaplaceBeltrami operator. Moreover, this kind of scheme preserves the mass.

1.2. Outline. The article is organized as follows. In Section 2, we first give the finitevolume notation associated with the particular geometry of the domain and the discrete unknowns and inner products. Section 3 is dedicated to introducing the finite-volume scheme and the associated energy estimates. In Section 4, we first prove the existence of a solution to the discrete scheme and then, we state a convergence theorem. The key-point is to obtain strong compactness for the approximate solutions and their traces. To this end, we introduce a new space translation operator and we prove a suitable space translation estimate which gives a limit in $L^{\infty}\left(0, T, H^{1}(\Omega)\right)$ whose trace is in $L^{\infty}\left(0, T, H^{1}(\Gamma)\right)$. Finally, in Section 5. we give numerical error estimates for the Cahn-Hilliard equation with dynamic boundary conditions. We also present qualitative results which are in agreement with the numerical simulations observed in the literature. 


\section{The Finite-Volume framework.}

2.1. Main notation. We recall that the domain $\Omega$ is not polygonal and that we have to solve an equation on the boundary $\Gamma$. Thus, the notation (see Fig. 2.1) are slightly different from the usual finite-volume notation (introduced for example in [10]).

Admissible mesh. We say that $\mathcal{T}$ is an admissible mesh of $\Omega$ if $\mathcal{T}$ is constituted of an interior mesh $\mathfrak{M}$ and a boundary mesh $\partial \mathfrak{M}$ satisfying the following properties.

- The interior mesh $\mathfrak{M}$ is given by a family of disjoint open subsets of $\Omega$ called control volumes and denoted by $\mathcal{K}$ such that,

$-\bar{\Omega}=\cup_{\mathcal{K} \in \mathfrak{M}} \mathcal{K}$;

- if $\mathcal{K}, \mathcal{L} \in \mathfrak{M}, \mathcal{K} \neq \mathcal{L}$, then ${ }^{\mathcal{K}} \cap \dot{\mathcal{L}}=\emptyset$;

- if $\mathcal{K}, \mathcal{L} \in \mathfrak{M}, \mathcal{K} \neq \mathcal{L}$ such that the dimension of $\overline{\mathcal{K}} \cap \overline{\mathcal{L}}$ is equal to 1 , then $\overline{\mathcal{K}} \cap \overline{\mathcal{L}}$ is the edge of the mesh separating the control volumes $\mathcal{K}$ and $\mathcal{L}$;

- for any $\mathcal{K} \in \mathfrak{M}$, we associate a point $x_{\mathcal{K}}$ such that if $\mathcal{K}, \mathcal{L}$ are two neighboring interior control volumes, the edge which separates $\mathcal{K}$ and $\mathcal{L}$ is orthogonal to the straight line going through $x_{\mathcal{K}}$ and $x_{\mathcal{L}}$.

- The boundary mesh $\partial \mathfrak{M}$ is the set of edges of the control volumes in $\mathfrak{M}$ included in $\Gamma$. We remark that these edges are not segments but curved sections. We note that the elements of $\partial \mathfrak{M}$ are both edges of control volumes in $\mathfrak{M}$ and boundary control volumes. Thus, when we consider them as control volumes belonging to $\partial \mathfrak{M}$ we note $\mathcal{L} \in \partial \mathfrak{M}$ and when we consider them as edges of an interior control volume we note $\sigma$. This mesh must also satisfies an orthogonality condition: for any $\mathcal{L} \in \partial \mathfrak{M}$ which is an edge of the interior control volume $\mathcal{K}$, we define $x_{\mathcal{L}}$ as the intersection between $\mathcal{L}$ and the straight line passing through $x_{\mathcal{K}}$ and orthogonal to the chord $\mathbf{e}_{\mathcal{L}}$ associated with $\mathcal{L}$. Then, $y_{\mathcal{L}}$ is the intersection between the chord $\mathbf{e}_{\mathcal{L}}$ and the line $\left(x_{\mathcal{K}} x_{\mathcal{L}}\right)$.

The set of edges. Let $\mathcal{E}$ be the set of edges of the mesh $\mathcal{T}, \mathcal{E}_{\text {int }}$ is the set of interior (flat) edges and $\mathcal{E}_{\text {ext }}$ is the set of exterior (curved) edges (we note that $\mathcal{E}_{\text {ext }}=\partial \mathfrak{M}$ ). Let $\mathcal{E}_{\mathcal{K}}$ be the set of edges of a given control volume $\mathcal{K} \in \mathfrak{M}$.

For any $\sigma \in \mathcal{E}$, we note

- $m_{\sigma}$ its length;

- $\sigma=\mathcal{K} \mid \mathcal{L}$ if $\sigma \in \mathcal{E}_{\text {int }}$ is the edge which separates the control volumes $\mathcal{K}$ and $\mathcal{L}$;

- $\mathcal{D}=\mathcal{D}_{\sigma}$ the quadrangle whose diagonals are the edge $\sigma$ and the line segment $\left[x_{\mathcal{K}}, x_{\mathcal{L}}\right]$ if $\sigma \in \mathcal{E}_{\text {int }}$;

- $\mathcal{D}=\mathcal{D}_{\sigma}=\left\{t x+(1-t) x_{\mathcal{K}}, t \in[0,1], x \in \sigma\right\}$ if $\sigma \in \mathcal{E}_{\text {ext }} \cap \mathcal{E}_{\mathcal{K}}$;

- $m_{\mathcal{D}}$ the Lebesgue measure of $\mathcal{D}$.

The interior mesh. We note that if $\mathcal{K}$ is a control volume with one edge, at least, on the boundary, then $\mathcal{K}$ is not polygonal and may be not convex.

For any $\mathcal{K} \in \mathfrak{M}$, we note:

- $\underline{\mathcal{K}}$ the polygon shaped by the vertices of $\mathcal{K}$; we remark that $\underline{\mathcal{K}}=\mathcal{K}$ if $\mathcal{E}_{\mathcal{K}} \cap \mathcal{E}_{\text {ext }}=\emptyset$ and that $\underline{\mathcal{K}}$ can be not included in $\Omega$ if $\mathcal{E}_{\mathcal{K}} \cap \mathcal{E}_{\text {ext }} \neq \emptyset$;

- $m_{\mathcal{K}}$ (respectively $m_{\underline{\mathcal{K}}}$ ) the Lebesgue measure of $\mathcal{K}$ (respectively $\underline{\mathcal{K}}$ ).

The boundary mesh. Let $\mathbf{e}_{\mathcal{L}}$ be the chord associated with $\mathcal{L}$. We note $m_{\mathcal{L}}$ (respectively $m_{\mathbf{e}_{\mathcal{L}}}$ ) the length of $\mathcal{L}$ (respectively $\mathbf{e}_{\mathcal{L}}$ ).

Let $\mathcal{V}$ be the set of vertices included in the boundary $\Gamma$ and $\mathcal{V}_{\mathcal{L}}$ is the set of vertices of the boundary control volume $\mathcal{L}$.

Distances and normals. For an edge $\sigma \in \mathcal{E}$, we note:

- $\overrightarrow{\mathbf{n}}_{\mathcal{K} \mathcal{L}}$ the unit normal vector to $\sigma$ going from $\mathcal{K}$ to $\mathcal{L}$ and $d_{\mathcal{K}, \mathcal{L}}$ the distance between $x_{\mathcal{K}}$ and $x_{\mathcal{L}}$ if $\sigma \in \mathcal{E}_{\text {int }}$; 
- $\overrightarrow{\mathbf{n}}_{\mathcal{K} \mathcal{L}}$ the unit normal vector to $\mathbf{e}_{\mathcal{L}}$ outward to $\mathcal{K}$ and $d_{\mathcal{K}, \mathcal{L}}$ the distance between $x_{\mathcal{K}}$ and $y_{\mathcal{L}}$ if $\sigma \in \mathcal{E}_{\text {ext }}$.

- $\overrightarrow{\mathbf{n}}_{\mathcal{K} \sigma}(x)$ the unit normal vector to $\sigma$ at the point $x \in \sigma$ outward to $\mathcal{K}$ (we remark that if $\sigma \in \mathcal{E}_{i n t}, \overrightarrow{\mathbf{n}}_{\mathcal{K} \sigma}(x)=\overrightarrow{\mathbf{n}}_{\mathcal{K} \mathcal{L}}$ for all $\left.x \in \sigma\right)$.

For a vertex $\mathbf{v}=\mathcal{L} \mid \mathcal{L}^{\prime}$ which separates the boundary control volumes $\mathcal{L}, \mathcal{L}^{\prime} \in \partial \mathfrak{M}$, we note

- $d_{\mathcal{L}, \mathbf{v}}$ the distance between the vertex $\mathbf{v}$ and the center $y_{\mathcal{L}}$;

- $d_{\mathcal{L}, \mathcal{L}^{\prime}}$ the sum of $d_{\mathcal{L}, \mathbf{v}}$ and $d_{\mathcal{L}^{\prime}, \mathbf{v}}$ : it is the approximation of the length $m_{\gamma_{\mathcal{L} \mathcal{L}^{\prime}}}$ of the $\operatorname{arc} \gamma_{\mathcal{L L}^{\prime}} \subset \Gamma$ passing through the vertex $\mathbf{v}=\mathcal{L} \mid \mathcal{L}^{\prime}$ and whose ends are $x_{\mathcal{L}}$ and $x_{\mathcal{L}^{\prime}}$;

- $n_{\mathbf{v} \mathcal{L}}$ gives an orientation of the curve $\gamma_{\mathcal{L L}^{\prime}}$ in function of the orientation of the curve $\Gamma: n_{\mathbf{v} \mathcal{L}}=1$ if the orientation of $\Gamma$ going from $\mathcal{L}$ to $\mathcal{L}^{\prime}$. Thus $n_{\mathbf{v} \mathcal{L}}= \pm 1$ and $n_{\mathbf{v} \mathcal{L}}=-n_{\mathbf{v} \mathcal{L}^{\prime}}$.

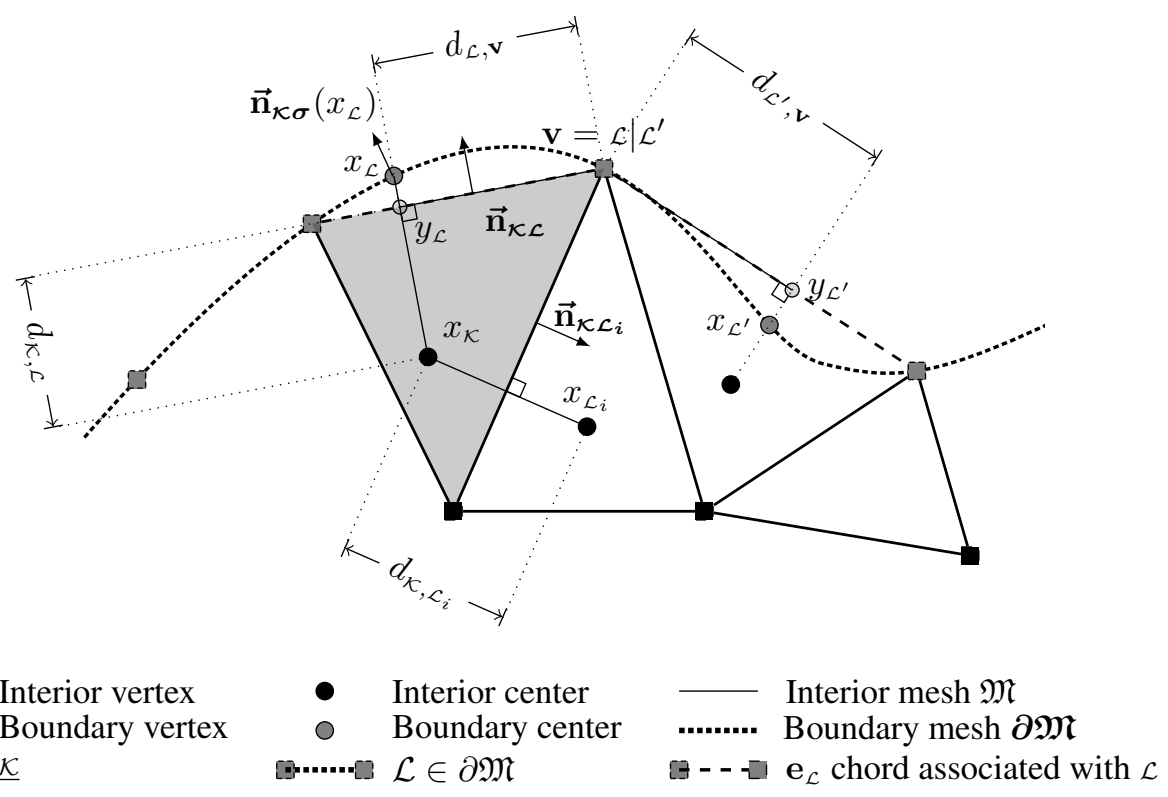

Fig. 2.1: Mesh $\mathcal{T}$ associated with $\Omega$

As regards the boundary mesh, we can remark that in the proposed scheme (Section 3.1) we only use the coordinates of the vertices of the mesh. All the other quantities are approximations and we do not use the equation of $\Gamma$. However, to pass to the limit in the convergence theorem we reason with the exact quantities. In the sequel (in particular in the proof of the convergence theorem) the following relations will be very useful and frequently use even if it is not expressly mentioned. For any $\mathcal{L} \in \partial \mathfrak{M}$,

$$
m_{\mathbf{e}_{\mathcal{L}}}-m_{\mathcal{L}}=\mathcal{O}\left(m_{\mathcal{L}}^{3}\right), \quad m_{\gamma_{\mathcal{L L}^{\prime}}}-d_{\mathcal{L}, \mathcal{L}^{\prime}}=\mathcal{O}\left(m_{\gamma_{\mathcal{L} \mathcal{L}^{\prime}}}\left(m_{\mathcal{L}}+m_{\mathcal{L}^{\prime}}\right)\right)
$$

and for any $\mathcal{K} \in \mathfrak{M}$ such that $\sigma=\mathcal{L} \in \mathcal{E}_{\mathcal{K}} \cap \mathcal{E}_{\text {ext }}$,

$$
m_{\underline{\mathcal{K}}}-m_{\mathcal{K}}=\mathcal{O}\left(\operatorname{diam}(\mathcal{K})^{3}\right), \quad\left|d\left(x_{\mathcal{K}}, x_{\mathcal{L}}\right)-d_{\mathcal{K}, \mathcal{L}}\right|=d\left(x_{\mathcal{L}}, y_{\mathcal{L}}\right)=\mathcal{O}\left(m_{\mathcal{L}}^{2}\right) .
$$

We can also note that for all $x \in \mathcal{L} \subset \Gamma, \overrightarrow{\mathbf{n}}(x)-\overrightarrow{\mathbf{n}}_{\mathcal{K} \mathcal{L}}=\mathcal{O}\left(m_{\mathcal{L}}\right)$. The proof of these results can be obtained by using the Taylor formulas and a parametrization of $\Gamma$. 
The mesh size is defined by $\operatorname{size}(\mathcal{T})=\sup \{\operatorname{diam}(\mathcal{K}), \mathcal{K} \in \mathfrak{M}\}$. We introduce a positive number $\operatorname{reg}(\mathcal{T})$ which measures the regularity of a given mesh,

$$
\operatorname{reg}(\mathcal{T}):=\max \left(\mathcal{N}, \max _{\substack{\mathcal{K} \in \mathfrak{M}_{\mathcal{K}} \\ \sigma \in \mathcal{E}_{\mathcal{K}}}} \frac{\operatorname{diam}(\mathcal{K})}{\operatorname{diam}\left(\mathcal{D}_{\sigma}\right)}, \max _{\mathcal{D}} \frac{\operatorname{diam}(\mathcal{D})}{\sqrt{m_{\mathcal{D}}}}, \max _{\mathcal{K} \in \mathfrak{M}} \frac{\operatorname{diam}(\mathcal{K})}{\sqrt{m_{\mathcal{K}}}}\right)
$$

where $\mathcal{N}$ is the maximum of edges incident to any vertex.

All the constants in the results below depends on this quantity which is very useful to perform the convergence analysis of finite-volume schemes. The number $\operatorname{reg}(\mathcal{T})$ must be uniformly bounded when the mesh size tends to 0 for the convergence results hold.

2.2. Discrete unknowns. Regarding the space discretization, we define the piecewise constant functions $u_{\mathfrak{M}} \in \mathbb{R}^{\mathfrak{M}}$ and $u_{\partial \mathfrak{M}} \in \mathbb{R}^{\partial \mathfrak{M}}$ as follows

$$
u_{\mathfrak{M}}=\sum_{\mathcal{K} \in \mathfrak{M}} u_{\mathcal{K}} 1_{\mathcal{K}} \in L^{\infty}(\Omega) \quad \text { and } \quad u_{\partial \mathfrak{M}}=\sum_{\mathcal{L} \in \partial \mathfrak{M}} u_{\mathcal{L}} 1_{\mathcal{L}} \in L^{\infty}(\Gamma),
$$

where $1_{\mathcal{K}}$ (respectively $1_{\mathcal{L}}$ ) is the indicator function of the control volume $\mathcal{K}$ (respectively $\mathcal{L}$ ). We also define the discrete function $u_{\mathcal{T}} \in \mathbb{R}^{\mathcal{T}}$ which we associate with the couple $u_{\mathcal{T}}=$ $\left(u_{\mathfrak{m}}, u_{\partial \mathfrak{M}}\right)$.

As regards the time discretization, we set $N \in \mathbb{N}^{*}$ and then $\Delta t=\frac{T}{N}$. For all $n \in\{1, \ldots, N\}$, we define $t^{n}=n \Delta t$. Then, we define $u_{\mathfrak{M}}^{\Delta t}$ (respectively $u_{\partial \mathfrak{M}}^{\Delta t}$ ) as the piecewise constant function in $(0, T) \times \Omega$ (respectively $(0, T) \times \Gamma)$ such that for any $t \in\left[t^{n}, t^{n+1}[\right.$,

$$
u_{\mathfrak{M}}^{\Delta t}(t, x)=u_{\mathcal{K}}^{n+1} \text { if } x \in \mathcal{K} \quad \text { and } \quad u_{\partial \mathfrak{M}}^{\Delta t}(t, x)=u_{\mathcal{L}}^{n+1} \text { if } x \in \mathcal{L}
$$

For a given time step $t^{n}$, the finite-volume scheme associates with each interior control volume $\mathcal{K} \in \mathfrak{M}$ an unknown value $c_{\mathcal{K}}^{n}$ and with each boundary control volume $\mathcal{L} \in \partial \mathfrak{M}$ an unknown value $c_{\mathcal{L}}^{n}$ for the concentration. Regarding the chemical potential, the same notation are used with an interior unknown $\mu_{\mathcal{K}}^{n}$ for all $\mathcal{K} \in \mathfrak{M}$ and a boundary unknown $\mu_{\mathcal{L}}^{n}$ for all $\mathcal{L} \in \partial \mathfrak{M}$. However the homogeneous Neumann boundary condition is associated with $\mu$, so we impose the value of the boundary unknowns $\mu_{\partial \mathfrak{M}}^{n} \in \mathbb{R}^{\partial \mathfrak{M}}$ as follows

$$
\mu_{\mathcal{L}}^{n}=\mu_{\mathcal{K}}^{n}, \quad \forall \mathcal{L} \in \partial \mathfrak{M} \text { such that } \mathcal{L}=\sigma \in \mathcal{E}_{\mathcal{K}} \cap \mathcal{E}_{\text {ext }} .
$$

2.3. Inner products and norms. Here, we define the inner products and the norms used in the paper. We define the discrete $L^{2}$-inner products and the discrete $H^{1}$-semi-definite inner products on $\mathbb{R}^{\mathcal{T}}$ and $\mathbb{R}^{\partial \mathfrak{M}}$.

DEFINITION 2.1 (Discrete $L^{2}$-inner products).

For any $u_{\mathfrak{M}}, v_{\mathfrak{M}} \in \mathbb{R}^{\mathfrak{M}}$ and for any $u_{\partial \mathfrak{M}}, v_{\partial \mathfrak{M}} \in \mathbb{R}^{\partial \mathfrak{M}}$, we define

$$
\left(u_{\mathfrak{M}}, v_{\mathfrak{M}}\right)_{\mathfrak{M}}=\sum_{\mathcal{K} \in \mathfrak{M}} m_{\underline{\mathcal{K}}} u_{\mathcal{K}} v_{\mathcal{K}} \quad \text { and } \quad\left(u_{\partial \mathfrak{M}}, v_{\partial \mathfrak{M}}\right)_{\partial \mathfrak{M}}=\sum_{\mathcal{L} \in \partial \mathfrak{M}} m_{\mathbf{e}_{\mathcal{L}}} u_{\mathcal{L}} v_{\mathcal{L}}
$$

The associated $L^{2}$-norms are noted $\|\cdot\|_{0, \mathfrak{m}}$ and $\|\cdot\|_{0, a \mathfrak{m}}$.

For any $u_{\mathfrak{M}} \in \mathbb{R}^{\mathfrak{M}}$ and for any $u_{\partial \mathfrak{M}} \in \mathbb{R}^{\partial \mathfrak{M}}$, we define the discrete $L^{p}$-norms on $\mathbb{R}^{\mathfrak{M}}$ and $\mathbb{R}^{\partial \mathfrak{M}}$ as follows

$$
\left\|u_{\mathfrak{M}}\right\|_{0, p, \mathfrak{M}}^{p}=\sum_{\mathcal{K} \in \mathfrak{M}} m_{\underline{\mathcal{K}}}\left|u_{\mathcal{K}}\right|^{p} \quad \text { and } \quad\left\|u_{\mathfrak{M}}\right\|_{0, p, \partial \mathfrak{M}}^{p}=\sum_{\mathcal{L} \in \partial \mathfrak{M}} m_{\mathbf{e}_{\mathcal{L}}}\left|u_{\mathcal{L}}\right|^{p}
$$

We can remark that the norm $\|\cdot\|_{0, p, \mathfrak{M}}$ (respectively $\|\cdot\|_{0, p, \partial \mathfrak{M}}$ ) is equivalent to the usual $L^{p}$ norm $\|\cdot\|_{L^{p}(\Omega)}^{p}=\sum_{\mathcal{K} \in \mathfrak{M}} m_{\mathcal{K}}\left|u_{\mathcal{K}}\right|^{p}$ in $\Omega$ (respectively $\|\cdot\|_{L^{p}(\Gamma)}^{p}=\sum_{\mathcal{L} \in \partial \mathfrak{M}} m_{\mathcal{L}}\left|u_{\mathcal{L}}\right|^{p}$ in $\Gamma$ ) with constants independent of the mesh size. 
DEFINITION 2.2 (Discrete $H^{1}$-semi-definite inner products).

- For any $u_{\mathcal{T}}, v_{\mathcal{T}} \in \mathbb{R}^{\mathcal{T}}$, we define the $H^{1}$-semi-definite inner product in $\mathbb{R}^{\mathcal{T}}$ as follows

$$
\llbracket u_{\mathcal{T}}, v_{\mathcal{T}} \rrbracket_{1, \mathcal{T}}=\sum_{\sigma=\mathcal{K} \mid \mathcal{\mathcal { L }} \in \mathcal{E}_{\text {int }}} \frac{m_{\sigma}}{d_{\mathcal{K}, \mathcal{L}}}\left(u_{\mathcal{K}}-u_{\mathcal{L}}\right)\left(v_{\mathcal{K}}-v_{\mathcal{L}}\right)+\sum_{\sigma=\mathcal{L} \in \mathcal{E}_{\text {ext }}} \frac{m_{\mathbf{e}_{\mathcal{L}}}}{d_{\mathcal{K}, \mathcal{L}}}\left(u_{\mathcal{K}}-u_{\mathcal{L}}\right)\left(v_{\mathcal{K}}-v_{\mathcal{L}}\right) .
$$

- For any $u_{\partial \mathfrak{M}}, v_{\partial \mathfrak{M}} \in \mathbb{R}^{\partial \mathfrak{M}}$, we define the $H^{1}$-semi-definite inner product in $\mathbb{R}^{\partial \mathfrak{M}}$

$$
\llbracket u_{\partial \mathfrak{M}}, v_{\partial \mathfrak{M}} \rrbracket_{1, \partial \mathfrak{M}}=\sum_{\mathbf{v}=\mathcal{L} \mid \mathcal{L}^{\prime} \in \mathcal{V}} d_{\mathcal{L}, \mathcal{L}^{\prime}}\left(\frac{u_{\mathcal{L}}-u_{\mathcal{L}^{\prime}}}{d_{\mathcal{L}, \mathcal{L}^{\prime}}}\right)\left(\frac{v_{\mathcal{L}}-v_{\mathcal{L}^{\prime}}}{d_{\mathcal{L}, \mathcal{L}^{\prime}}}\right) .
$$

The associated $H^{1}$-seminorms are noted $|\cdot|_{1, \mathcal{T}}$ and $|\cdot|_{1, \partial \mathfrak{M}}$.

From this, for any $u_{\mathcal{T}} \in \mathbb{R}^{\mathcal{T}}, u_{\partial \mathfrak{M}} \in \mathbb{R}^{\partial \mathfrak{M}}$ we can define the $H^{1}$-norms as follows

$$
\left\|u_{\mathcal{T}}\right\|_{1, \mathcal{T}}^{2}=\left\|u_{\mathfrak{M}}\right\|_{0, \mathfrak{M}}^{2}+\left|u_{\mathcal{T}}\right|_{1, \mathcal{T}}^{2} \text { and }\left\|u_{\partial \mathfrak{M}}\right\|_{1, \partial \mathfrak{M}}^{2}=\left\|u_{\partial \mathfrak{M}}\right\|_{0, \partial \mathfrak{M}}^{2}+\left|u_{\partial \mathfrak{M}}\right|_{1, \partial \mathfrak{M}}^{2} .
$$

Moreover, we have to define norms in time and space.

DEFINITION 2.3 (Discrete norms in time and space).

Let $\mathcal{N}$ be a discrete norm on a space $\mathcal{B}$, then we define

- The discrete $L^{p}(0, T ; \mathcal{N}(\mathcal{B}))$ norm by

$$
\left\|u^{\Delta t}\right\|_{L^{p}(0, T ; \mathcal{N}(\mathcal{B}))}=\left(\sum_{n=0}^{N-1} \Delta t\left(\mathcal{N}\left(u^{n+1}\right)\right)^{p}\right)^{\frac{1}{p}} .
$$

- The discrete $L^{\infty}(0, T ; \mathcal{N}(\mathcal{B}))$ norm by

$$
\left\|u^{\Delta t}\right\|_{L^{\infty}(0, T ; \mathcal{N}(\mathcal{B}))}=\sup _{n \leq N} \mathcal{N}\left(u^{n}\right) .
$$

\section{The Finite-Volume scheme and the discrete energy.}

\subsection{The numerical scheme.}

DEFINITION 3.1 (Discrete mean projection).

Let $u$ be an integrable function on $\Omega$ which admits a trace $u_{1 \Gamma}$ integrable on $\Gamma$, we set $\mathbb{P}_{\mathcal{T}}^{m} u=$ $\left(\mathbb{P}_{\mathfrak{M}}^{m} u, \mathbb{P}_{\partial \mathfrak{M}}^{m} u_{1 \Gamma}\right)$ with

$$
\mathbb{P}_{\mathfrak{M}}^{m} u=\left(\frac{1}{m_{\mathcal{K}}} \int_{\mathcal{K}} u(x) \mathrm{d} x\right)_{\mathcal{K} \in \mathfrak{M}} \quad \text { and } \quad \mathbb{P}_{\partial \mathfrak{M}}^{m} u_{\mid \Gamma}=\left(\frac{1}{m_{\mathcal{L}}} \int_{\mathcal{L}} u_{\mid \Gamma}(x) \mathrm{d} \sigma(x)\right)_{\mathcal{L} \in \partial \mathfrak{M}} .
$$

With this definition, choosing $c^{0} \in H_{\Gamma}^{1}(\Omega)$ (where $H_{\Gamma}^{1}(\Omega)$ is defined by (1.10), we can define the discrete initial concentration as follows,

$$
c_{\mathcal{\tau}}^{0}=\mathbb{P}_{\mathcal{T}}^{m} c^{0} .
$$

In order to obtain the finite-volume scheme associated with the Cahn-Hilliard model (1.7), we have to integrate the continuous equations. We integrate equations (1.1) for $t \in\left[t^{n}, t^{n+1}\right]$ and for $\mathcal{K} \in \mathfrak{M}$. For Laplace operators, we use a consistent two-point flux approximation together with the homogeneous Neumann boundary condition (1.3) for the equation (1.1a). The dynamic boundary condition (1.4) is integrated for $t \in\left[t^{n}, t^{n+1}\right]$ and for $\mathcal{L} \in \partial \mathfrak{M}$. We use a consistent two-point flux approximation for the Laplace-Beltrami operator on $\Gamma$.

With respect to the non-linear terms, in the paper we use a semi-implicit discretization described in Subsection 3.3. We denote by $d^{f_{b}}$ (respectively $d^{f_{s}}$ ) the discretization of the potential $f_{b}$ (respectively $f_{s}$ ). 
The scheme we propose reads: For any $n \geq 0$, find $\left(c_{\mathcal{T}}^{n+1}, \mu_{\mathcal{T}}^{n+1}\right) \in \mathbb{R}^{\mathcal{T}} \times \mathbb{R}^{\mathcal{T}}$ such that for any $u_{\mathcal{T}}, v_{\mathcal{T}} \in \mathbb{R}^{\mathcal{T}}$,

$$
\left\{\begin{aligned}
\left(\frac{c_{\mathfrak{M}}^{n+1}-c_{\mathfrak{M}}^{n}}{\Delta t}, v_{\mathfrak{M}}\right)_{\mathfrak{M}}= & -\Gamma_{b} \llbracket \mu_{\mathcal{T}}^{n+1}, v_{\mathcal{T}} \rrbracket_{1, \mathcal{T}}, \\
\left(\mu_{\mathfrak{M}}^{n+1}, u_{\mathfrak{M}}\right)_{\mathfrak{M}}= & \mathcal{A}_{\Delta} \sum_{\sigma=\mathcal{K} \mid \mathcal{L} \in \mathcal{E}_{\text {int }}} \frac{m_{\sigma}}{d_{\mathcal{K}, \mathcal{L}}}\left(c_{\mathcal{K}}^{n+1}-c_{\mathcal{L}}^{n+1}\right)\left(u_{\mathcal{K}}-u_{\mathcal{L}}\right) \\
& +\mathcal{A}_{\Delta} \sum_{\sigma=\mathcal{L} \in \mathcal{E}_{\text {ext }}} \frac{m_{\mathbf{e}_{\mathcal{L}}}}{d_{\mathcal{K}, \mathcal{L}}}\left(c_{\mathcal{K}}^{n+1}-c_{\mathcal{L}}^{n+1}\right) u_{\mathcal{K}} \\
& +\mathcal{A}_{f_{b}} \sum_{\mathcal{K} \in \mathfrak{M}} m_{\underline{\mathcal{K}}} d^{f_{b}}\left(c_{\mathcal{K}}^{n}, c_{\mathcal{K}}^{n+1}\right) u_{\mathcal{K}}, \\
\mathcal{A}_{\partial t}\left(\frac{\left.c_{\partial \mathfrak{M}}^{n+1}-c_{\partial \mathfrak{M}}^{n}, u_{\partial \mathfrak{M}}\right)_{\partial \mathfrak{M}}=}{\Delta t}\right. & -\mathcal{A}_{\Delta_{\Gamma}} \llbracket c_{\partial \mathfrak{M}}^{n+1}, u_{\partial \mathfrak{M}} \rrbracket_{1, \partial \mathfrak{M}} \\
& -\mathcal{A}_{f_{s}} \sum_{\mathcal{L} \in \partial \mathfrak{M}} m_{\mathbf{e}_{\mathcal{L}}} d^{f_{s}}\left(c_{\mathcal{L}}^{n}, c_{\mathcal{L}}^{n+1}\right) u_{\mathcal{L}} \\
& -\mathcal{A}_{\Delta} \sum_{\sigma=\mathcal{L} \in \mathcal{E}_{\text {ext }}} \frac{m_{\mathbf{e}_{\mathcal{L}}}}{d_{\mathcal{K}, \mathcal{L}}}\left(c_{\mathcal{L}}^{n+1}-c_{\mathcal{K}}^{n+1}\right) u_{\mathcal{L}} .
\end{aligned}\right.
$$

We notice that we write the scheme in a "variational" formulation which is equivalent to the classical finite-volume formulation and which will be more useful in the analysis.

Let us remark that in equation $3.2 \mathrm{a}$ ) the finite-volume approximation of the term $\Delta \mu$ only uses the interior edges of the mesh $\mathfrak{M}$ while in equation $(3.2 \mathrm{~b}$ ) the approximation of the term $\Delta c$ uses all the edges of the mesh (interior and exterior). The reason for this is that $\mu$ satisfies the homogeneous Neumann boundary condition (so the exterior edges do not step in) while $c$ satisfies the dynamic boundary condition (1.4) where the exterior edges intervene (this term is essential to allow the coupling with equation (3.2c) on the boundary mesh $\partial \mathfrak{M}$ ). Setting $v_{\mathcal{T}} \equiv 1$ in equation $3.2 \mathrm{a}$, we note that we have the conservation of the volume at the discrete level,

$$
\sum_{\mathcal{K} \in \mathfrak{M}} m_{\underline{\mathcal{K}}} c_{\mathcal{K}}^{n}=\sum_{\mathcal{K} \in \mathfrak{M}} m_{\underline{\mathcal{K}}} c_{\mathcal{K}}^{0}, \quad \forall n \in\{1, \ldots, N\} .
$$

3.2. Energy estimates. We define the discrete free energy associated with the continuous free energy (1.8). As in the continuous case, the discrete free energy is decomposed into a bulk contribution $\mathcal{F}_{b, \mathcal{T}}$ and a surface contribution $\mathcal{F}_{s, \partial \mathfrak{M}}$ : For any $c_{\mathcal{T}} \in \mathbb{R}^{\mathcal{T}}$, we set

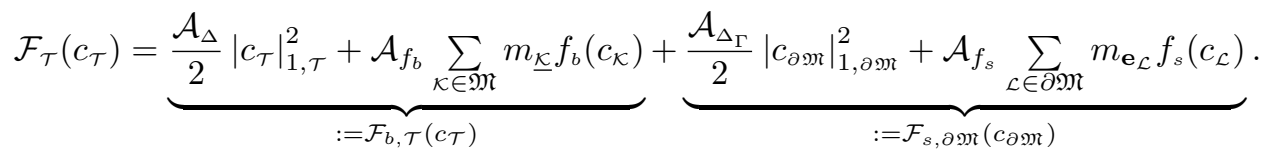

Regardless of the choice of the discretization of non-linear terms, we have a general energy estimate.

PROPOSITION 3.2 (General energy equality).

Let $c_{\mathcal{T}}^{n} \in \mathbb{R}^{\mathcal{T}}$. We assume that there exists a solution $\left(c_{\mathcal{T}}^{n+1}, \mu_{\mathcal{T}}^{n+1}\right)$ to discrete Problem (3.2). Then, the following equality holds

$$
\begin{aligned}
\mathcal{F}_{\mathcal{T}}\left(c_{\mathcal{T}}^{n+1}\right)- & \mathcal{F}_{\mathcal{T}}\left(c_{\mathcal{T}}^{n}\right)+\Delta t \Gamma_{b}\left|\mu_{\mathcal{T}}^{n+1}\right|_{1, \mathcal{T}}^{2}+\frac{\mathcal{A}_{\partial t}}{\Delta t}\left\|c_{\partial \mathfrak{M}}^{n+1}-c_{\partial \mathfrak{M}}^{n}\right\|_{0, \partial \mathfrak{M}}^{2} \\
& +\frac{\mathcal{A}_{\Delta}}{2}\left|c_{\mathcal{T}}^{n+1}-c_{\mathcal{T}}^{n}\right|_{1, \mathcal{T}}^{2}+\frac{\mathcal{A}_{\Delta_{\Gamma}}}{2}\left|c_{\partial \mathfrak{M}}^{n+1}-c_{\partial \mathfrak{M}}^{n}\right|_{1, \partial \mathfrak{M}}^{2} \\
= & \mathcal{A}_{f_{b}} \sum_{\mathcal{K} \in \mathfrak{M}} m_{\mathcal{\mathcal { K }}}\left(f_{b}\left(c_{\mathcal{K}}^{n+1}\right)-f_{b}\left(c_{\mathcal{K}}^{n}\right)-d^{f_{b}}\left(c_{\mathcal{K}}^{n}, c_{\mathcal{K}}^{n+1}\right)\left(c_{\mathcal{K}}^{n+1}-c_{\mathcal{K}}^{n}\right)\right) \\
& +\mathcal{A}_{f_{s}} \sum_{\mathcal{L} \in \partial \mathfrak{M}} m_{\mathbf{e}_{\mathcal{L}}}\left(f_{s}\left(c_{\mathcal{L}}^{n+1}\right)-f_{s}\left(c_{\mathcal{L}}^{n}\right)-d^{f_{s}}\left(c_{\mathcal{L}}^{n}, c_{\mathcal{L}}^{n+1}\right)\left(c_{\mathcal{L}}^{n+1}-c_{\mathcal{L}}^{n}\right)\right) .
\end{aligned}
$$


Proof. We consider the scheme 3.2) with $u_{\mathcal{T}}=c_{\mathcal{T}}^{n+1}-c_{\mathcal{T}}^{n}$ and $v_{\mathcal{T}}=-\Delta t \mu_{\mathcal{T}}^{n+1}$ as test functions and we add the three equations.

The definition of the discrete energy cannot give a discrete counterpart to the equality (1.9) which gives the dissipation of the continuous energy. However, with a good choice for the discretization of non-linear terms, we can obtain the dissipation of the discrete energy.

3.3. Discretization of non-linear terms. In order to obtain an energy estimate without any condition on the time step $\Delta t$, we choose a time discretization for the non-linear terms such that the right hand side of (3.4) is equal to 0 . Namely, we set

$$
d^{f_{b}}(x, y)=\frac{f_{b}(y)-f_{b}(x)}{y-x} \quad \text { and } \quad d^{f_{s}}(x, y)=\frac{f_{s}(y)-f_{s}(x)}{y-x}, \quad \forall x, y, x \neq y .
$$

In practice, we mostly used polynomial functions for the potentials $f_{b}$ and $f_{s}$. Then, the terms $d^{f_{b}}(x, y)$ and $d^{f_{s}}(x, y)$ can be written as polynomial functions in the variables $x, y$. Thus, we do not have numerical instability when $x$ is too close from $y$.

In effect, we have

$$
d^{f_{b}}(x, y)=f_{b}^{\prime}\left(\frac{x+y}{2}\right)+(x-y)^{2} P(x, y)
$$

where $P$ is a polynomial function in the variables $x, y$.

Thus, we remark that $d^{f_{b}}(x, x)=f_{b}^{\prime}(x)$ and $d^{f_{s}}$ satisfies the same properties.

PROPOSITION 3.3 (Discrete free energy equality).

Let $c_{\mathcal{T}}^{n} \in \mathbb{R}^{\mathcal{T}}$. We assume that there exists a solution $\left(c_{\mathcal{T}}^{n+1}, \mu_{\mathcal{T}}^{n+1}\right)$ to discrete Problem (3.2). Then, the following equality holds

$$
\begin{aligned}
\mathcal{F}_{\mathcal{T}}\left(c_{\mathcal{T}}^{n+1}\right)-\mathcal{F}_{\mathcal{T}}\left(c_{\mathcal{T}}^{n}\right) & +\Delta t \Gamma_{b}\left|\mu_{\mathcal{T}}^{n+1}\right|_{1, \mathcal{T}}^{2}+\frac{\mathcal{A}_{\partial t}}{\Delta t}\left\|c_{\partial \mathfrak{M}}^{n+1}-c_{\partial \mathfrak{M}}^{n}\right\|_{0, \partial \mathfrak{M}}^{2} \\
& +\frac{\mathcal{A}_{\Delta}}{2}\left|c_{\mathcal{T}}^{n+1}-c_{\mathcal{T}}^{n}\right|_{1, \mathcal{T}}^{2}+\frac{\mathcal{A}_{\Delta_{\Gamma}}}{2}\left|c_{\partial \mathfrak{M}}^{n+1}-c_{\partial \mathfrak{M}}^{n}\right|_{1, \partial \mathfrak{M}}^{2}=0 .
\end{aligned}
$$

Remark: We can also use a fully implicit discretization for non-linear terms, namely

$$
d^{f_{b}}\left(c_{\mathcal{K}}^{n}, c_{\mathcal{K}}^{n+1}\right)=f_{b}^{\prime}\left(c_{\mathcal{K}}^{n+1}\right), \forall \mathcal{K} \in \mathfrak{M} \quad \text { and } \quad d^{f_{s}}\left(c_{\mathcal{L}}^{n}, c_{\mathcal{L}}^{n+1}\right)=f_{s}^{\prime}\left(c_{\mathcal{L}}^{n+1}\right), \forall \mathcal{L} \in \partial \mathfrak{M} .
$$

In this case, we obtain the dissipation of the discrete energy for any $\Delta t \leq \Delta t_{0}$ where $\Delta t_{0}$ only depends on the parameters of the equation. All the results given in the paper are true for this discretization if we assume that the time step satisfies $\Delta t \leq \Delta t_{0}$.

4. Existence and convergence theorems. The existence and convergence results proved below are true for other choices of discretization for the non-linear terms. Thus, we give here general assumptions (which are satisfied by the semi-implicit discretization) of the discretization of the non-linear potential $d^{f_{b}}$ to obtain these results: $d^{f_{b}}$ is of $\mathcal{C}^{1}$ class and there exist $C_{b} \geq 0$ and a real $p$ such that $2 \leq p<+\infty$,

$$
\begin{aligned}
\left|d^{f_{b}}(a, b)\right| & \leq C_{b}\left(1+|a|^{p-1}+|b|^{p-1}\right), \\
\left|D\left(d^{f_{b}}(a, .)\right)(b)\right| & \leq C_{b}\left(1+|a|^{p-2}+|b|^{p-2}\right) .
\end{aligned}
$$

4.1. Preliminary results. In this subsection, we consider a bounded connected Lipschitz domain $\Omega \subset \mathbb{R}^{2}$ and we denote by $\Gamma=\partial \Omega$ its boundary. Let $\mathcal{T}$ be an admissible mesh associated with $\Omega$ as described in the subsection 2.1. 
First, we recall the discrete Poincaré inequality and the discrete Poincaré-Sobolev inequality which will be very useful in the sequel. The proofs can be found in [2] in the case where $\Omega$ is polygonal and with Neumann boundary conditions but these results can be easily adapted to our case.

LEMMA 4.1 (Poincaré inequality,[2, Theorem 5]).

There exists $C_{1}>0$ depending only on $\Omega$ and $\operatorname{reg}(\mathcal{T})$ such that

$$
\left\|u_{\mathfrak{M}}-m_{\mathfrak{M}}\left(u_{\mathfrak{M}}\right)\right\|_{0, \mathfrak{M}} \leq G_{1}\left|u_{\mathcal{T}}\right|_{1, \mathcal{T}}, \quad \forall u_{\mathcal{T}} \in \mathbb{R}^{\mathcal{T}}
$$

where $m_{\mathfrak{M}}\left(u_{\mathfrak{M}}\right)=\frac{1}{M_{\Omega}} \sum_{\mathcal{K} \in \mathfrak{M}} m_{\underline{\mathcal{K}}} u_{\mathcal{K}}$ and $M_{\Omega}=\sum_{\mathcal{K} \in \mathfrak{M}} m_{\underline{\mathcal{K}}}$.

This Lemma gives for any $u_{\mathcal{T}} \in \mathbb{R}^{\bar{\tau}}$,

$$
\left\|u_{\mathcal{T}}\right\|_{1, \mathcal{T}}^{2} \leq\left(2 C_{1}^{2}+1\right)\left|u_{\mathcal{T}}\right|_{1, \mathcal{T}}^{2}+2 M_{\Omega}\left(m_{\mathfrak{M}}\left(u_{\mathfrak{M}}\right)\right)^{2} .
$$

LEMMA 4.2 (Poincaré-Sobolev inequality,[2, Theorem 3]).

Let $1 \leq q<+\infty$, then there exists $C_{2}>0$ depending only on $q$, $\Omega$ and $\operatorname{reg}(\mathcal{T})$ such that

$$
\left\|u_{\mathfrak{M}}\right\|_{0, q, \mathfrak{M}} \leq C_{2}\left\|u_{\mathcal{T}}\right\|_{1, \mathcal{T}}, \quad \forall u_{\mathcal{T}} \in \mathbb{R}^{\mathcal{T}}
$$

Now, we give a Sobolev inequality for the one dimension manifold $\Gamma$.

LEMmA 4.3. There exists $C_{3}>0$ depending only on $\Gamma$ and $\operatorname{reg}(\mathcal{T})$ such that

$$
\left\|u_{\partial \mathfrak{M}}\right\|_{L^{\infty}(\Gamma)} \leq G_{3}\left\|u_{\partial \mathfrak{M}}\right\|_{1, \partial \mathfrak{M}} .
$$

Proof. Let $\mathcal{L}_{1} \in \partial \mathfrak{M}$, then

$$
u_{\mathcal{L}_{1}}=\left(u_{\mathcal{L}_{1}}-\frac{1}{M_{\Gamma}} \sum_{\mathcal{L} \in \partial \mathfrak{M}} m_{\mathbf{e}_{\mathcal{L}}} u_{\mathcal{L}}\right)+\frac{1}{M_{\Gamma}} \sum_{\mathcal{L} \in \partial \mathfrak{M}} m_{\mathbf{e}_{\mathcal{L}}} u_{\mathcal{L}} \quad \text { with } M_{\Gamma}=\sum_{\mathcal{L} \in \partial \mathfrak{M}} m_{\mathbf{e}_{\mathcal{L}}} .
$$

The Cauchy-Schwarz inequality implies

$$
\left|u_{\mathcal{L}_{1}}\right|^{2} \leq \frac{2}{M_{\Gamma}}\left(\sum_{\mathcal{L} \in \partial \mathfrak{M}} m_{\mathbf{e}_{\mathcal{L}}}\left|u_{\mathcal{L}_{1}}-u_{\mathcal{L}}\right|^{2}+\left\|u_{\partial \mathfrak{M}}\right\|_{0, \partial \mathfrak{M}}^{2}\right)
$$

If $u_{\mathcal{L}_{1}}, u_{\mathcal{L}_{2}} \in \partial \mathfrak{M}$, the triangle inequality and the Cauchy-Schwarz inequality give

$$
\left|u_{\mathcal{L}_{1}}-u_{\mathcal{L}_{2}}\right| \leq\left(\sum_{\mathbf{v}=\mathcal{L} \mid \mathcal{L}^{\prime} \in \mathcal{V}} d_{\mathcal{L}, \mathcal{L}^{\prime}}\right)^{\frac{1}{2}}\left|u_{\partial \mathfrak{M}}\right|_{1, \partial \mathfrak{M}}
$$

Thus, there exists $C_{\Gamma}>0$ depending only on $\Gamma$ such that

$$
\left|u_{\mathcal{L}_{1}}\right|^{2} \leq 2\left(C_{\Gamma}\left|u_{\partial \mathfrak{M}}\right|_{1, \partial \mathfrak{M}}^{2}+\frac{1}{M_{\Gamma}}\left\|u_{\partial \mathfrak{M}}\right\|_{0, \partial \mathfrak{M}}^{2}\right)
$$

and the proof is complete.

4.2. Existence theorem. This subsection is devoted to state general existence theorem. THEOREM 4.4 (Existence of a discrete solution).

Let $c_{\mathcal{T}}^{n} \in \mathbb{R}^{\mathcal{T}}$. We assume that:

- the potentials $f_{b}$ and $f_{b}$ satisfy dissipativity assumption (1.5) and the bulk potential satisfies the growth condition (1.6); 
- the discretization of non-linear terms satisfies growth condition (4.1) for $f_{b}$.

Then, there exists at least one solution $\left(c_{\mathcal{T}}^{n+1}, \mu_{\mathcal{T}}^{n+1}\right) \in \mathbb{R}^{\mathcal{T}} \times \mathbb{R}^{\mathcal{T}}$ to Problem (3.2).

Proof.

The proof is very similar to the one given in [4, Theorem 2.9], thus we do not give the details here. The key-point is the use of the topological degree theory [8].

As regards the a priori estimates, we have three key-points.

- We consider the Problem (3.2) with $\delta d^{f_{b}}$ (respectively $\delta d^{f_{s}}$ ) instead of $d^{f_{b}}$ (respectively $\left.d^{f_{s}}\right)$ with $\delta \in[0,1]$.

- Using the energy estimate [3.5, the quantities $\left\|c_{\mathcal{T}}^{n+1}\right\|_{1, \mathcal{T}},\left\|c_{\partial \mathfrak{M}}^{n+1}\right\|_{1, \partial \mathfrak{M}}$ and $\left|\mu_{\mathcal{T}}^{n+1}\right|_{1, \mathcal{T}}$ are bounded independently of $\delta$.

- Choosing $u_{\mathcal{T}} \equiv 1$ as test function in the equations $(3.2 \mathrm{~b})$ and $(3.2 \mathrm{c})$, we obtain a bound (independent of $\delta$ ) on the mean-value of $\mu_{\mathcal{T}}^{n+1}$ and thanks to the Poincaré estimate (4.3), we have the expected bound on $\left\|\mu_{\mathcal{T}}^{n+1}\right\|_{1, \mathcal{T}}$.

The proof of the well-posedness of the scheme when $\delta=0$ is classical.

4.3. The convergence theorem. In order to give the convergence theorem, we have to recall the definition of a solution to the continuous equation (1.7) in a weak sense.

DEFINITION 4.5 (Weak formulation).

We say that a couple $(c, \mu) \in L^{\infty}\left(0, T ; H^{1}(\Omega)\right) \times L^{2}\left(0, T ; H^{1}(\Omega)\right)$ such that $c_{1 \Gamma} \in L^{\infty}\left(0, T ; H^{1}(\Gamma)\right)$ is solution to continuous Problem 1.77 in the weak sense if for all $\phi \in \mathcal{C}_{c}^{2}\left(\mathbb{R} \times \mathbb{R}^{2}\right)$ such that $\phi(T,)=$.0 , the following identities hold

$$
\begin{aligned}
& \int_{0}^{T} \int_{\Omega}\left(-\partial_{t} \phi c+\Gamma_{b} \nabla \mu \cdot \nabla \phi\right) \mathrm{d} x \mathrm{~d} t=\int_{\Omega} c^{0} \phi(0, .) \mathrm{d} x \\
& \int_{0}^{T} \int_{\Omega}\left(-\mu \phi+\mathcal{A}_{\Delta} \nabla c \cdot \nabla \phi+\mathcal{A}_{f_{b}} f_{b}^{\prime}(c) \phi\right) \mathrm{d} x \mathrm{~d} t \\
& \quad+\int_{0}^{T} \int_{\Gamma}\left(-\mathcal{A}_{\partial t} \partial_{t} \phi c_{1 \Gamma}+\mathcal{A}_{\Delta_{\Gamma}} \nabla_{\Gamma} c_{\mid \Gamma} \cdot \nabla_{\Gamma} \phi+\mathcal{A}_{f_{s}} f_{s}^{\prime}\left(c_{\mid \Gamma}\right) \phi\right) \mathrm{d} \sigma(x) \mathrm{d} t \\
& \quad=\mathcal{A}_{\partial t} \int_{\Gamma} \operatorname{Tr}\left(c^{0}\right) \phi(0, .) \mathrm{d} \sigma(x) .
\end{aligned}
$$

THEOREM 4.6 (Convergence theorem).

Let $c^{0} \in H_{\Gamma}^{1}(\Omega)$ (see definition 1.10) and $\left(\left(\left(c_{\mathcal{T}}^{\Delta t}\right)^{(m)}\right),\left(\left(\mu_{\mathcal{T}}^{\Delta t}\right)^{(m)}\right)\right)_{m \in \mathbb{N}}$ a sequence of solutions to Problem (3.2) associated with a sequence of discretizations such that the space and time steps, $\operatorname{size}\left(\mathcal{T}^{(m)}\right)$ and $\Delta t^{(m)}$ respectively, tend to 0 . Then, assuming that $\operatorname{reg}\left(\mathcal{T}^{(m)}\right)$ is bounded when $m \rightarrow+\infty$, there exists a weak solution $(c, \mu)$ to Problem (1.7) (in the sense of Definition 4.5) for the initial data $c^{0}$ such that, up to a subsequence, the following convergence properties hold, for all $q \geq 1$

$$
\begin{gathered}
\left(c_{\mathcal{T}}^{\Delta t}\right)^{(m)} \rightarrow c \text { in } L^{2}\left(0, T ; L^{q}(\Omega)\right), \quad\left(c_{\partial \mathfrak{M}}^{\Delta t}\right)^{(m)} \rightarrow c_{1 \Gamma} \text { in } L^{2}\left(0, T ; L^{q}(\Gamma)\right), \\
\text { and }\left(\mu_{\mathcal{T}}^{\Delta t}\right)^{(m)} \rightarrow \mu \text { in } L^{2}\left(0, T ; L^{q}(\Omega)\right) \text { weakly. }
\end{gathered}
$$

Because of non-linearities in the equation both in $\Omega$ and on $\Gamma$, to prove this theorem we need strong compactness both in $L^{2}((0, T) \times \Omega)$ and in $L^{2}((0, T) \times \Gamma)$. Thus, we have to apply the Kolmogorov theorem to obtain the existence of the limit and the strong convergences. Then, we can pass to the limit in the scheme and, especially in the non-linear terms. To apply the Kolmogorov theorem we have to apply three key elements: the bounds on the discrete 
solutions (see Proposition 4.11), an estimate of space translates (see Theorem 4.20) and an estimate of time translates (see Theorem 4.26.

4.3.1. Properties of the mean-value projection. Definition (3.1) of the discrete initial concentration induce us to give the following properties on the discrete mean-value projection which will be useful in the sequel.

PROPOSITION 4.7 ([1, Proposition 3.5]).

For any $p \geq 1$, there exists $C_{4}>0$ independent of $\operatorname{size}(\mathcal{T})$ such that

$$
\left\|\mathbb{P}_{\mathfrak{M}}^{m} u\right\|_{0, p, \mathfrak{M}} \leq C^{\mathbb{4}}\|u\|_{L^{p}(\Omega)}, \quad \forall u \in L^{p}(\Omega) .
$$

Assumption A: Let $\mathcal{P} \subset \mathbb{R}^{2}$ be a pseudo-triangle with one curved side $\sigma \subset \mathbb{R}^{2}$. There exist $\nu_{1}, \nu_{2}>0$ such that for any sub-arc $\tilde{\sigma} \subset \sigma$, the corresponding sub-triangle $\mathcal{P}_{\tilde{\sigma}}$ (see Fig.4.1p satisfies,

$$
\nu_{1} \leq \frac{m_{\mathcal{P}_{\tilde{\sigma}}}}{m_{\tilde{\sigma}}} \leq \nu_{2}
$$
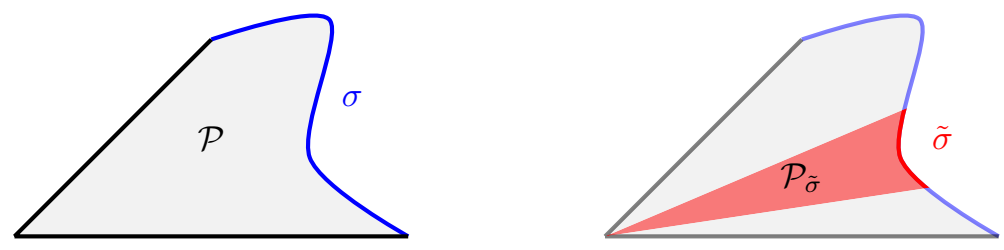

Fig. 4.1: The pseudo-triangle $\mathcal{P}$ and one of its sub-triangles

LEMma 4.8 ([10, Lemma 3.4] and [3]).

For any $p \geq 1$, there exists $C_{5}>0$ depending only on $\operatorname{reg}(\mathcal{T}), \nu_{1}, \nu_{2}$ and $p$ such that,

- for any segment $\sigma \subset \mathbb{R}^{2}$ and for any bounded set $\mathcal{P} \subset \mathbb{R}^{2}$ with positive measure,

- for any pseudo-triangle $\mathcal{P} \subset \mathbb{R}^{2}$ with one curved side $\sigma \subset \mathbb{R}^{2}$ which satisfies the Assumption A,

and for any $u \in H^{1}\left(\mathbb{R}^{2}\right)$, then

$$
\left|u_{\mathcal{P}}-u_{\sigma}\right|^{p} \leq C_{5} \frac{\left(m_{\sigma}+\operatorname{diam}(\mathcal{Q})\right)^{p}}{m_{\mathcal{P}}} \int_{\mathcal{Q}}|\nabla u(z)|^{p} \mathrm{~d} z,
$$

where $u_{\mathcal{P}}$ denotes the mean-value of $u$ on $\mathcal{P}, u_{\sigma}$ the mean-value of $u$ on $\sigma$ and

- $\mathcal{Q}=\widehat{\mathcal{P}_{\sigma}}$ is the convex hull of $\mathcal{P} \cup \sigma$ if $\sigma$ is a segment;

- $\mathcal{Q}=\mathcal{P}$ if $\sigma \subset \partial \mathcal{P}$ is the curved edge of the pseudo-triangle $\mathcal{P}$.

In this study, we want to apply this lemma (specifically the second point) to the control volumes $\mathcal{K} \in \mathfrak{M}$ with one edge belongs to $\Gamma$ (namely it is a curved edge). Let us remark that we can prove that for a small enough mesh size the assumption A is satisfied for these control volumes (see [3]).

Remark: This lemma is crucial to prove the proposition below which will be used in the proof of Proposition 4.11 which yields the bounds on the discrete solutions. These bounds are one of the key point of the proof of the convergence Theorem 4.6

Indeed, to obtain the bounds on the discrete solutions we have to project the initial data such that the discrete $H^{1}$-norms on $\Omega$ and $\Gamma$ are both controlled by the $H_{\Gamma}^{1}(\Omega)$-norm of the initial 
data. If $\sigma$ is a segment (the first point of the lemma) the proof of this result is classical and can be found, for instance in [10, Lemma 3.4]. Thus, we can think that we can avoid to use the case where $\sigma$ is a curved edge, whose proof is more complicated, by choosing the mean on the chords for the initial data. However, with this choice, we lose the $H^{1}$-estimate on the boundary. In fact, in this case we are not able to prove the Proposition 4.10 which allows to obtain the $H^{1}$-estimate on the boundary. For this reason, we choose the discrete initial concentration equals to the mean projection on the curved edges (see definition (3.1) of $c_{\mathcal{T}}^{0}$ ). Thus, in this case we need to use the second point on the Lemma 4.8 which is a technical result whose proof is given in [3].

Proposition 4.9. There exists $C_{6}>0$ independent of $\operatorname{size}(\mathcal{T})$ such that for any function $u \in H^{1}(\Omega)$,

$$
\left|\mathbb{P}_{\mathcal{T}}^{m} u\right|_{1, \mathcal{T}} \leq C_{6} \mid\|\nabla u\|_{L^{2}(\Omega)} .
$$

Proof. Lemma 4.8 gives,

$$
\begin{aligned}
\left|\mathbb{P}_{\mathcal{T}}^{m} u\right|_{1, \mathcal{T}}^{2} \leq & 2 C_{\mathfrak{G}} \sum_{\sigma=\mathcal{K} \mid \mathcal{L} \in \mathcal{E}_{\text {int }}} \frac{m_{\sigma}}{d_{\mathcal{K}, \mathcal{L}}}\left(\frac{4 \operatorname{diam}(\widehat{\mathcal{K}})^{2}}{m_{\mathcal{K}}} \int_{\widehat{\mathcal{K}}}|\nabla u(z)|^{2} \mathrm{~d} z+\frac{4 \operatorname{diam}(\widehat{\mathcal{L}})^{2}}{m_{\mathcal{L}}} \int_{\widehat{\mathcal{L}}}|\nabla u(z)|^{2} \mathrm{~d} z\right) \\
& +C_{\mathfrak{5}} \sum_{\sigma=\mathcal{L} \in \mathcal{E}_{\text {ext }}} \frac{m_{\mathbf{e}_{\mathcal{L}}}}{d_{\mathcal{K}, \mathcal{L}}} \frac{\left(m_{\sigma}+\operatorname{diam}(\mathcal{K})\right)^{2}}{m_{\mathcal{K}}} \int_{\mathcal{K}}|\nabla u(z)|^{2} \mathrm{~d} z .
\end{aligned}
$$

Then, thanks to definition (2.1) of $\operatorname{reg}(\mathcal{T})$ we conclude the proof.

Proposition 4.10. Let $u \in H^{1}(\Gamma)$, there exists $C_{7}>0$ independent of $\operatorname{size}(\mathcal{T})$ such that,

$$
\left|\mathbb{P}_{\partial \mathfrak{M}}^{m} u\right|_{1, \partial \mathfrak{M}} \leq C_{\mathbf{Z}}\left\|\nabla_{\Gamma} u\right\|_{L^{2}(\Gamma)} .
$$

Proof. We consider two neighboring boundary control volumes $\mathcal{L}, \mathcal{L}^{\prime} \in \partial \mathfrak{M}$, then

$$
\left|\mathbb{P}_{\mathcal{L}}^{m} u-\mathbb{P}_{\mathcal{L}^{\prime}}^{m} u\right|^{2} \leq \frac{1}{m_{\mathcal{L}} m_{\mathcal{L}^{\prime}}} \int_{\mathcal{L}} \int_{\mathcal{L}^{\prime}}\left|\int_{\widehat{x y}} \nabla_{\Gamma} u(z) \cdot \overrightarrow{\boldsymbol{\tau}}(z) \mathrm{d} \sigma(z)\right|^{2} \mathrm{~d} \sigma(x) \mathrm{d} \sigma(y),
$$

where $\vec{\tau}$ is the unit tangent vector to the curve $\Gamma$.

Thanks to the Cauchy-Schwarz inequality, we have

$$
\left|\mathbb{P}_{\mathcal{L}}^{m} u-\mathbb{P}_{\mathcal{L}^{\prime}}^{m} u\right|^{2} \leq\left(m_{\mathcal{L}}+m_{\mathcal{L}^{\prime}}\right) \int_{\mathcal{L} \cup \mathcal{L}^{\prime}}\left|\nabla_{\Gamma} u(z)\right|^{2} \mathrm{~d} \sigma(z),
$$

and the mesh regularity completes the proof.

4.3.2. Bounds of the solutions. The following proposition is one of the key points of the proof of convergence.

PROPOSITION 4.11 (Bounds of the discrete solutions).

Assuming that the assumptions of Theorem 4.6 are satisfied. Then, there exist positive constants $M_{1}, M_{2}, M_{3}, M_{4}$ and $M_{5}$ independent of $\Delta t$ and $\operatorname{size}(\mathcal{T})$ such that,

$$
\begin{gathered}
\sup _{n \leq N}\left\|c_{\mathcal{T}}^{n}\right\|_{1, \mathcal{T}} \leq M_{1}, \quad \sup _{n \leq N}\left\|c_{\partial \mathfrak{M}}^{n}\right\|_{1, \partial \mathfrak{M}} \leq M_{2}, \\
\sum_{n=0}^{N-1} \Delta t\left\|\mu_{\mathfrak{M}}^{n+1}\right\|_{1, \mathcal{T}}^{2} \leq M_{3}, \quad \sum_{n=0}^{N-1} \Delta t\left\|\frac{c_{\mathcal{T}}^{n+1}-c_{\mathcal{T}}^{n}}{\Delta t}\right\|_{1, \mathcal{T}}^{2} \leq \frac{M_{4}}{\Delta t} \\
\text { and } \quad \sum_{n=0}^{N-1} \Delta t\left\|\frac{c_{\partial \mathfrak{M}}^{n+1}-c_{\partial \mathfrak{M}}^{n}}{\Delta t}\right\|_{0, \partial \mathfrak{M}}^{2}+\Delta t^{2} \sum_{n=0}^{N-1}\left|\frac{c_{\partial \mathfrak{M}}^{n+1}-c_{\partial \mathfrak{M}}^{n}}{\Delta t}\right|_{1, \partial \mathfrak{M}}^{2} \leq M_{5} .
\end{gathered}
$$




\section{Proof.}

- The discrete energy estimate (3.5) gives a uniform bound on the discrete free energy,

$$
\forall n \in \llbracket 0, N \rrbracket, \quad \mathcal{F}_{\mathcal{T}}\left(c_{\mathcal{T}}^{n}\right) \leq \mathcal{F}_{\mathcal{T}}\left(c_{\mathcal{T}}^{0}\right)
$$

Then, thanks to the polynomial growth assumption 1.6 , we have

$$
\begin{aligned}
\mathcal{F}_{\mathcal{T}}\left(c_{\mathcal{T}}^{0}\right) \leq & \frac{\mathcal{A}_{\Delta}}{2}\left|c_{\mathcal{T}}^{0}\right|_{1, \mathcal{T}}^{2}+\mathcal{A}_{f_{b}} C_{b}\left(M_{\Omega}+\left\|c_{\mathcal{T}}^{0}\right\|_{0, p, \mathfrak{M}}^{p}\right) \\
& +\frac{\mathcal{A}_{\Delta_{\Gamma}}}{2}\left|c_{\partial \mathfrak{M}}^{0}\right|_{1, \partial \mathfrak{M}}^{2}+\mathcal{A}_{f_{s}} C_{\Gamma} \max _{\bar{B}\left(0,\left\|c^{0}\right\|_{L^{\infty}(\Gamma)}\right)}\left|f_{s}\right|
\end{aligned}
$$

Thus, definition (3.1) of $c_{\mathcal{T}}^{0}$ and Propositions 4.7, 4.9 and 4.10 imply that there exists $K_{0}>0$ such that,

$$
\mathcal{F}_{\mathcal{T}}\left(c_{\mathcal{T}}^{0}\right) \leq K_{0}
$$

Thanks to dissipativity assumption (1.5),

$$
\begin{aligned}
\mathcal{F}_{\mathcal{T}}\left(c_{\mathcal{T}}^{n}\right) \geq & \frac{\mathcal{A}_{\Delta}}{2}\left|c_{\mathcal{T}}^{n}\right|_{1, \mathcal{T}}^{2}+\mathcal{A}_{f_{b}} \alpha_{1}\left\|c_{\mathfrak{M}}^{n}\right\|_{0, \mathfrak{M}}^{2}-\mathcal{A}_{f_{b}} \alpha_{2} M_{\Omega} \\
& +\frac{\mathcal{A}_{\Delta_{\Gamma}}}{2}\left|c_{\partial \mathfrak{M}}^{n}\right|_{1, \partial \mathfrak{M}}^{2}+\mathcal{A}_{f_{s}} \alpha_{1}\left\|c_{\partial \mathfrak{M}}^{n}\right\|_{0, \partial \mathfrak{M}}^{2}-\mathcal{A}_{f_{s}} \alpha_{2} M_{\Gamma}
\end{aligned}
$$

Using (4.8, 4.9) and 4.10) and setting $K_{1}:=K_{0}+\alpha_{2}\left(\mathcal{A}_{f_{b}} M_{\Omega}+\mathcal{A}_{f_{s}} M_{\Gamma}\right)>0$, there exist positive constants $K_{2}, K_{3}, K_{4}$ and $K_{5}$ such that for all $n \in \llbracket 0, N \rrbracket$,

$$
\begin{array}{rlrl}
\left\|c_{\mathfrak{M}}^{n}\right\|_{0, \mathfrak{M}}^{2} & \leq \frac{K_{1}}{\mathcal{A}_{f_{b}} \alpha_{1}}:=K_{2}, & \left|c_{\mathcal{T}}^{n}\right|_{1, \mathcal{T}}^{2} \leq \frac{2 K_{1}}{\mathcal{A}_{\Delta}}:=K_{[3}, \\
\left\|c_{\partial \mathfrak{M}}^{n}\right\|_{0, \partial \mathfrak{M}}^{2} \leq \frac{K_{1}}{\mathcal{A}_{f_{s}} \alpha_{1}}:=K_{[4}, & \left|c_{\mathcal{T}}^{n}\right|_{1, \partial \mathfrak{M}}^{2} \leq \frac{2 K_{1}}{\mathcal{A}_{\Delta_{\Gamma}}}:=K_{[5} .
\end{array}
$$

These estimates are established for all $n \in \llbracket 0, N \rrbracket$, thus

$$
\begin{gathered}
\sup _{n \leq N}\left\|c_{\mathcal{T}}^{n}\right\|_{1, \mathcal{T}} \leq \sqrt{K_{2}+K_{[3}}:=M_{1}, \\
\sup _{n \leq N}\left\|c_{\mathcal{T}}^{n}\right\|_{1, \partial \mathfrak{M}} \leq \sqrt{K_{4}+K_{[5}}:=M_{2} .
\end{gathered}
$$

- Adding energy estimates 3.5 for $n$ from 0 to $N-1$, then

$$
\begin{aligned}
\mathcal{F}_{\mathcal{T}}\left(c_{\mathcal{T}}^{N}\right) & +\sum_{n=0}^{N-1}\left(\Delta t \Gamma_{b}\left|\mu_{\mathcal{T}}^{n+1}\right|_{1, \mathcal{T}}^{2}+\frac{\mathcal{A}_{\partial t}}{\Delta t}\left\|c_{\partial \mathfrak{M}}^{n+1}-c_{\partial \mathfrak{M}}^{n}\right\|_{0, \partial \mathfrak{M}}^{2}\right. \\
& \left.+\frac{\mathcal{A}_{\Delta}}{2}\left|c_{\mathcal{T}}^{n+1}-c_{\mathcal{T}}^{n}\right|_{1, \mathcal{T}}^{2}+\frac{\mathcal{A}_{\Delta_{\Gamma}}}{2}\left|c_{\partial \mathfrak{M}}^{n+1}-c_{\partial \mathfrak{M}}^{n}\right|_{1, \partial \mathfrak{M}}^{2}\right)=\mathcal{F}_{\mathcal{T}}\left(c_{\mathcal{T}}^{0}\right) .
\end{aligned}
$$

Thanks to estimates 4.9 and 4.10, we have

$$
\sum_{n=0}^{N-1} \Delta t\left|\mu_{\mathcal{T}}^{n+1}\right|_{1, \mathcal{T}}^{2} \leq \frac{K_{1}}{\Gamma_{b}} \text { and } \sum_{n=0}^{N-1} \frac{1}{\Delta t}\left\|c_{\partial \mathfrak{M}}^{n+1}-c_{\partial \mathfrak{M}}^{n}\right\|_{0, \partial \mathfrak{M}}^{2} \leq \frac{K_{1}}{\mathcal{A}_{\partial t}}
$$


Setting $u_{\mathcal{T}} \equiv 1$ in scheme $(3.2)$ and subtracting equations $(3.2 \mathrm{~b})$ and $(3.2 \mathrm{c})$, then for all $n \in \llbracket 0, N-1 \rrbracket$,

$$
\begin{aligned}
\sum_{\mathcal{K} \in \mathfrak{M}} m_{\underline{\mathcal{K}}} \mu_{\mathcal{K}}^{n+1}= & \mathcal{A}_{f_{b}} \sum_{\mathcal{K} \in \mathfrak{M}} m_{\underline{\mathcal{K}}} d^{f_{b}}\left(c_{\mathcal{K}}^{n}, c_{\mathcal{K}}^{n+1}\right)+\mathcal{A}_{f_{s}} \sum_{\mathcal{L} \in \partial \mathfrak{M}} m_{\mathbf{e}_{\mathcal{L}}} d^{f_{s}}\left(c_{\mathcal{L}}^{n}, c_{\mathcal{L}}^{n+1}\right) \\
& +\frac{\mathcal{A}_{\partial t}}{\Delta t} \sum_{\mathcal{L} \in \partial \mathfrak{M}} m_{\mathbf{e}_{\mathcal{L}}}\left(c_{\mathcal{L}}^{n+1}-c_{\mathcal{L}}^{n}\right) .
\end{aligned}
$$

Using polynomial growth assumption (4.1), Lemma 4.2 and bound 4.11), there exists $K_{6}>0$ such that

$$
\sum_{\mathcal{K} \in \mathfrak{M}} m_{\underline{\mathcal{K}}}\left|d^{f_{b}}\left(c_{\mathcal{K}}^{n}, c_{\mathcal{K}}^{n+1}\right)\right| \leq C_{b}\left(M_{\Omega}+2\left(M_{1}\right)^{p-1} C_{2}^{p-1}\right):=K_{[6},
$$

As regards the surface potential $f_{s}$, there exists $K_{7}>0$ such that,

$$
\left|d^{f_{s}}\left(c_{\mathcal{L}}^{n}, c_{\mathcal{L}}^{n+1}\right)\right| \leq \max _{\bar{B}\left(0, M_{2}\right)}\left|f_{s}^{\prime}\right|:=K_{\square}, \quad \forall \mathcal{L} \in \partial \mathfrak{M} .
$$

Then, applying Poincaré inequality (4.3) to $\mu_{\mathcal{T}}^{n+1}$, there exists a positive constant $K_{8}:=\frac{4}{M_{\Omega}}\left(\mathcal{A}_{f_{b}} K_{6}+\mathcal{A}_{f_{s}} K_{7}\right)^{2}$ such that by using 4.13, we finally obtain

$$
\sum_{n=0}^{N-1} \Delta t\left\|\mu_{\mathfrak{M}}^{n+1}\right\|_{1, \tau}^{2} \leq\left(2 C_{\square}^{2}+1\right) \frac{K_{\underline{1}}}{\Gamma_{b}}+\frac{4 \mathcal{A}_{\partial t} M_{\Gamma} K_{\square}}{M_{\Omega}}+T K_{\square}:=M_{3} .
$$

- Using (4.9), (4.10) and (4.12), we deduce

$$
\sum_{n=0}^{N-1}\left|c_{\mathcal{T}}^{n+1}-c_{\mathcal{T}}^{n}\right|_{1, \mathcal{T}}^{2} \leq \frac{2 K_{[1}}{\mathcal{A}_{\Delta}} .
$$

The discrete form of the volume conservation 3.3 gives $m_{\mathfrak{M}}\left(c_{\mathfrak{M}}^{n+1}-c_{\mathfrak{M}}^{n}\right)=0$, so thanks to Poincaré inequality 4.2,

$$
\sum_{n=0}^{N-1}\left\|\frac{c_{\mathcal{T}}^{n+1}-c_{\mathcal{T}}^{n}}{\Delta t}\right\|_{1, \mathcal{T}}^{2} \leq \frac{1}{\Delta t^{2}}\left(1+C_{\text {田}}\right) \frac{2 K_{\square}}{\mathcal{A}_{\Delta}}:=\frac{M_{4}}{\Delta t^{2}} .
$$

- In the same way, setting $M_{5}=\frac{K_{\square}}{\mathcal{A}_{\partial t}}+\frac{2 K_{\square}}{\mathcal{A}_{\Delta_{\Gamma}}}$ we obtain

$$
\sum_{n=0}^{N-1}\left\|\frac{c_{\partial \mathfrak{M}}^{n+1}-c_{\partial \mathfrak{M}}^{n}}{\Delta t}\right\|_{0, \partial \mathfrak{M}}^{2}+\Delta t \sum_{n=0}^{N-1}\left|\frac{c_{\partial \mathfrak{M}}^{n+1}-c_{\partial \mathfrak{M}}^{n}}{\Delta t}\right|_{1, \partial \mathfrak{M}}^{2} \leq \frac{M_{5}}{\Delta t} .
$$

and the claim is proved.

4.3.3. Weak convergence of the discrete gradient. In this subsection, we give the definition of the discrete gradient and a result of weak convergence used in the proof of Theorem 4.6

DEFINITION 4.12 (Discrete gradient).

We define the discrete gradient operator $\nabla^{\mathcal{T}}: \mathbb{R}^{\mathcal{T}} \rightarrow\left(\mathbb{R}^{2}\right)^{\mathcal{E}}$ as follows: For any $u_{\mathcal{T}} \in \mathbb{R}^{\mathcal{T}}$,

$$
\nabla^{\mathcal{\tau}} u_{\mathcal{T}}=\sum_{\sigma \in \mathcal{E}} 1_{\mathcal{D}} \nabla_{\mathcal{D}}^{\mathcal{T}} u_{\mathcal{T}} \in\left(L^{2}(\Omega)\right)^{2}, \quad \text { with } \nabla_{\mathcal{D}}^{\mathcal{\tau}} u_{\mathcal{T}}=2 \frac{u_{\mathcal{L}}-u_{\mathcal{K}}}{d_{\mathcal{K}, \mathcal{L}}} \overrightarrow{\mathbf{n}}_{\mathcal{K} \mathcal{L}}, \quad \forall \sigma \in \mathcal{E},
$$


where $1_{\mathcal{D}}$ is the indicator function of the diamond cell $\mathcal{D}$.

PROPOSITION 4.13 (Weak convergence of the discrete gradient, see [11, Lemma 2]). Let $\left(u_{\mathcal{T}^{(m)}}\right)_{m \in \mathbb{N}}$ be a sequence of discrete functions in $\mathbb{R}^{\mathcal{T}^{(m)}}$ associated with a sequence of discretizations such that the mesh size $\operatorname{size}\left(\mathcal{T}^{(m)}\right)$ tends to 0 (with $\operatorname{reg}\left(\mathcal{T}^{(m)}\right)$ bounded). We assume that there exists $M>0$ independent of $\operatorname{size}\left(\mathcal{T}^{(m)}\right)$ such that

$$
\left\|u_{\mathcal{T}^{(m)}}\right\|_{1, \mathcal{T}^{(m)}} \leq M, \quad \forall m \geq 0 .
$$

Then, up to a subsequence, $\left(u_{\mathcal{T}^{(m)}}\right)_{m \in \mathbb{N}}$ weakly converges in $L^{q}(\Omega)(q \geq 1)$ towards a certain $u \in H^{1}(\Omega)$ and $\left(\nabla^{\mathcal{T}} u_{\mathcal{T}^{(m)}}\right)_{m \in \mathbb{N}}$ weakly converges towards $\nabla u$ in $L^{2}$.

DEFINITION 4.14 (Discrete tangential gradient).

For any $u_{\partial \mathfrak{M}} \in \mathbb{R}^{\partial \mathfrak{M}}$, we define the discrete tangential gradient $\nabla_{\Gamma}^{\partial \mathfrak{M}}: \mathbb{R}^{\partial \mathfrak{M}} \rightarrow \mathbb{R}^{\mathcal{V}}$ by

$$
\nabla_{\Gamma}^{\partial \mathfrak{M}} u_{\partial \mathfrak{M}}=\left(\nabla_{\Gamma, \mathbf{v}}^{\partial \mathfrak{M}} u_{\partial \mathfrak{M}}\right)_{\mathbf{v} \in \mathcal{V}} \text { with } \nabla_{\Gamma, \mathbf{v}}^{\partial \mathfrak{M}} u_{\partial \mathfrak{M}}=\frac{u_{\mathcal{L}^{\prime}}-u_{\mathcal{L}}}{d_{\mathcal{L}, \mathcal{L}^{\prime}}} n_{\mathbf{v} \mathcal{L}}, \forall \mathbf{v}=\mathcal{L} \mid \mathcal{L}^{\prime} \in \mathcal{V}
$$

4.3.4. Time and space translates of approximate solutions. In order to pass to the limit in the scheme, because of the non-linearities, we have to use strong compactness in the domain $\Omega$ but also on its boundary $\Gamma$. Thus, we have to establish estimations of time and space translates that will enable us to obtain the convergence of a sequence of solution $\left(\left(c_{\mathcal{T}}^{\Delta t}\right)^{(m)}\right)_{m \in \mathbb{N}}$ to discrete Problem 3.2 towards a certain $c$ in $L^{2}((0, T) \times \Omega)$ and those of $\left(\left(c_{\partial \mathfrak{M}}^{\Delta t}\right)^{(m)}\right)_{m \in \mathbb{N}}$ towards $\operatorname{Tr} c$ in $L^{2}((0, T) \times \Gamma)$.

To this end, we build a particular discrete extension operator on a neighborhood of $\bar{\Omega}$.

DEFINITION 4.15 (Projection on the boundary).

We consider the locally Lipschitz continuous map

$$
\psi:\left(x_{\Gamma}, s\right) \in \Gamma \times \mathbb{R}_{+}^{*} \mapsto x_{\Gamma}+s \vec{n}\left(x_{\Gamma}\right) \in \mathbb{R}^{2},
$$

where $\vec{n}\left(x_{\Gamma}\right)$ is the outward unit normal vector to $\Omega$ at the point $x_{\Gamma} \in \Gamma$.

There exists $\alpha>0$ such that $\psi$ is a Lipschitz diffeomorphism from $\Gamma \times] 0, \alpha\left[\right.$ onto $\Omega_{\alpha}=\{x \in$ $\left.\Omega^{c}, d(x, \Gamma)<\alpha\right\}=\psi(\Gamma \times] 0, \alpha[)$ with $\alpha<\frac{2}{\|\kappa\|_{L^{\infty}}}$ (where $\kappa$ is the curvature of $\Gamma$ ). Then, for any $x \in \Omega_{\alpha}$ the projection on the boundary $\mathbb{P}_{\Gamma} x \in \Gamma$ is well defined: $\mathbb{P}_{\Gamma} x$ is the unique point on $\Gamma$ such that $\left|\mathbb{P}_{\Gamma} x-x\right|=d(x, \Gamma)$ and for any $x \in \Omega_{\alpha}, \psi\left(\mathbb{P}_{\Gamma} x, d(x, \Gamma)\right)=x$.

To build the extension operator associated with a mesh $\mathcal{T}$ of $\Omega$, we consider the open set $\Omega_{\alpha}$. Noting that in the continuous case, we can define the extension operator $P: H_{\Gamma}^{1}(\Omega) \rightarrow$ $H^{1}\left(\mathbb{R}^{2}\right)$ as follows,

$$
P u(x)=\left\{\begin{array}{cl}
u(x) & \text { if } x \in \Omega \\
\operatorname{Tr} u\left(\mathbb{P}_{\Gamma} x\right) \theta(d(x, \Gamma)) & \text { if } x \in \Omega_{\alpha} \\
0 & \text { otherwise }
\end{array}\right.
$$

where $\theta \in \mathcal{C}_{c}^{\infty}(\mathbb{R})$ satisfies $\theta(0)=1$ and $\theta(\alpha)=0$.

We follow the idea of the continuous case to build the discrete extension operator.

We first define a mesh $\mathfrak{M}_{\alpha}$ of the open set $\Omega_{\alpha}$ (see Fig. 4.2):

- Setting $\tilde{n}_{\alpha}=\frac{\alpha}{\operatorname{size}(\mathcal{T})}$ then if we note $E$ the floor function we can introduce,

$$
n_{\alpha}=\left\{\begin{array}{cr}
\tilde{n}_{\alpha} & \text { if } \tilde{n}_{\alpha} \in \mathbb{N} \\
E\left(\tilde{n}_{\alpha}\right)+1 & \text { if } \tilde{n}_{\alpha} \notin \mathbb{N}
\end{array} \text { and } \quad h_{\alpha}=\frac{\alpha}{n_{\alpha}} \leq \operatorname{size}(\mathcal{T}) .\right.
$$


- For any $\mathcal{L} \in \partial \mathfrak{M}$, we define $n_{\alpha}$ control volumes as follows,

$$
\mathcal{R}_{\mathcal{L}}^{i}=\psi(\mathcal{L} \times](i-1) h_{\alpha}, i h_{\alpha}[), \quad \forall i \in\left\{1, \cdots, n_{\alpha}\right\} .
$$

At each control volume, we associate the point $x_{\mathcal{R}_{\mathcal{L}}^{i}}=\psi\left(\left\{x_{\mathcal{L}}\right\} \times\left\{\left(i-\frac{1}{2}\right) h_{\alpha}\right\}\right)$ called the center of the control volume $\mathcal{R}_{\mathcal{L}}^{i}$.

- Let $\mathcal{E}_{\text {int }}^{\alpha}$ be the set of interior edges of the mesh $\mathfrak{M}_{\alpha}$ and $\mathcal{E}_{\text {ext }}^{\alpha}$ is the set of exterior edges which do not belong to $\mathcal{E}_{\text {ext }}$. The set $\mathcal{E}_{i n t}^{\alpha}$ is then split into two subsets:

- $\mathcal{E}_{\alpha_{\|}}$is the set of edges which separate two control volumes $\mathcal{R}_{\mathcal{L}}^{i}$ and $\mathcal{R}_{\mathcal{L}}^{i+1}$ (for $i \in\left\{1, \cdots, n_{\alpha}-1\right\}$ ) associated with the same boundary control volume $\mathcal{L} \in \partial \mathfrak{M}$. For any edge $\sigma \in \mathcal{E}_{\alpha_{\|}}$, we note $\sigma=\sigma_{\mathcal{L}}^{i, i+1}$ the edge which separates the control volumes $\mathcal{R}_{\mathcal{L}}^{i}$ and $\mathcal{R}_{\mathcal{L}}^{i+1}$ and $m_{\sigma_{f}^{i, i+1}}$ its length. Noting that these edges are curved sections, we denote by $\mathbf{e}_{\mathcal{L}}^{i, i+1}$ the chord associated with $\sigma_{\mathcal{L}}^{i, i+1}$ and $m_{\mathbf{e}_{\mathcal{L}}^{i, i+1}}$ its length.

- $\mathcal{E}_{\alpha_{\perp}}$ is the set of edges which separate a control volume $\mathcal{R}_{\mathcal{L}}^{i}$ (associated with $\mathcal{L} \in \partial \mathfrak{M}$ ) and a control volume $\mathcal{R}_{\mathcal{C}^{\prime}}$ (associated with $\mathcal{L}^{\prime} \in \partial \mathfrak{M}$ ) where $\mathcal{L}, \mathcal{L}^{\prime}$ are two neighboring cells. For an edge $\sigma \in \mathcal{E}_{\alpha_{\perp}}$ which separates the control volumes $\mathcal{R}_{\mathcal{L}}^{i}$ and $\mathcal{R}_{\mathcal{L}^{\prime}}^{i}$, we note $\gamma_{\mathcal{R}^{\prime}}^{i}$ the arc passing through the centers $x_{\mathcal{R}_{\mathcal{L}}^{i}}$ and $x_{\mathcal{R}_{\mathcal{L}^{\prime}}^{i}}$, namely $\gamma_{\mathcal{R}_{\mathcal{R}^{\prime}}}^{i}=\psi\left(\gamma_{\mathcal{L L}^{\prime}} \times\left\{\left(i-\frac{1}{2}\right) h_{\alpha}\right\}\right)$ and we note $m_{\gamma_{\mathcal{R}^{\prime}}^{i}}$ its length.

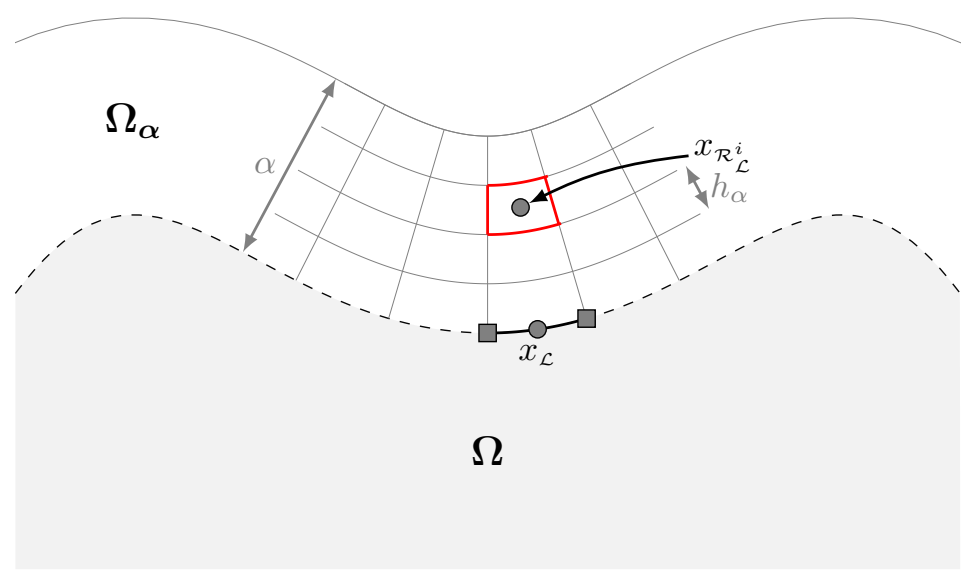

Fig. 4.2: Definition of the mesh $\mathfrak{M}_{\alpha}$

We can note that this mesh is only defined for the needs of the proof and, from a numerical point of view, we never build it.

We can link the length of the edges of the mesh $\mathfrak{M}_{\alpha}$ and those of the mesh $\partial \mathfrak{M}$ by the following identity.

Proposition 4.16. Let $\gamma$ be an arc included in $\Gamma$ and $\varsigma$ the arc defined by $\varsigma=\psi(\gamma \times$ $\{\delta\})$ with $\delta \in[0, \alpha]$. Thus, by denoting $\kappa$ the curvature of $\Gamma$ the following identity holds,

$$
\left|m_{\varsigma}-m_{\gamma}\right| \leq \delta\|\kappa\|_{L^{\infty}} m_{\gamma} .
$$

The proof of this result is obtained by considering the function $\psi_{\delta}(t)=\varphi(t)+\delta \overrightarrow{\mathbf{n}}(\varphi(t))$ where $\varphi$ is an arc-length parametrization of the curve $\Gamma$. 
We define the mesh $\mathcal{T}_{\alpha}=\mathcal{T} \cup \mathfrak{M}_{\alpha}$ and we note $\mathcal{K} \in \mathcal{T}_{\alpha}$ its control volumes.

DEFINITION 4.17 (Space extension operator). Let $\Psi_{\mathcal{T}}^{\Omega_{\alpha}}: \mathbb{R}^{\mathcal{T}} \rightarrow \mathbb{R}^{2}$ be the extension operator defined as follows

$$
\Psi_{\mathcal{T}}^{\Omega_{\alpha}}\left(u_{\mathcal{T}}\right)(x)=\left\{\begin{array}{cl}
u_{\mathcal{T}} & \text { if } x \in \Omega, \\
u_{\mathcal{R}_{\mathcal{L}}^{i}}=u_{\mathcal{L}} \theta\left(d\left(x_{\mathcal{R}_{\mathcal{L}}^{i}}, \Gamma\right)\right) & \text { if } x \in \mathcal{R}_{\mathcal{L}}^{i} \in \mathfrak{M}_{\alpha},
\end{array}\right.
$$

where $\theta \in \mathcal{C}_{c}^{\infty}\left(\left[0, \alpha[)\right.\right.$ and satisfies $\theta(x)=1$ for all $x \in\left[0, \frac{\alpha}{2}\right]$.

In the mesh $\mathfrak{M}_{\alpha}$ there are curved edges which complicates the understanding of the results. Thus, we would like to use a polygonal mesh whose edges are segments. To this end, we use the mesh $\mathcal{T}_{\alpha}$ to construct a polygonal mesh $\mathcal{T}_{\alpha}^{P}$. As for the mesh $\mathcal{T}$, for any $\mathcal{K} \in \mathcal{T}_{\alpha}$ we note $\underline{\mathcal{K}}$ the polygon shaped by the vertices of $\mathcal{K}$. Then, we can define the mesh $\mathcal{T}_{\alpha}^{P}$ as the union, on all the control volumes of $\mathcal{T}_{\alpha}$, of the control volumes $\underline{\mathcal{K}}$. The center of the control volume $\underline{\mathcal{K}}$ is the same of the one associated with the control volume $\mathcal{K}$, thus we note it $x_{\mathcal{K}}$. We define $\Omega^{P}=\bigcup_{\mathcal{K} \in \mathfrak{M}} \underline{\mathcal{K}}$ and $\Omega_{\alpha}^{P}=\bigcup_{\mathcal{K} \in \mathfrak{M}_{\alpha}} \underline{\mathcal{K}}$ the polygonal approximations of $\Omega$ and $\Omega_{\alpha}$.

Noting that there exists $C_{\alpha, \Gamma}>0$ independent of $\operatorname{size}(\mathcal{T})$ such that $\operatorname{size}\left(\mathcal{T}_{\alpha}^{P}\right) \leq C_{\alpha, \Gamma} \operatorname{size}(\mathcal{T})$.

DEFINITION 4.18.

We define the operator $\zeta^{\tau_{\alpha}}: \mathbb{R}^{\tau_{\alpha}} \rightarrow \mathbb{R}^{\tau_{\alpha}^{P}}$ such that, for any $u_{\tau_{\alpha}} \in \mathbb{R}^{\tau_{\alpha}}$, we have

$$
\zeta^{\mathcal{T}_{\alpha}}\left(u_{\mathcal{T}_{\alpha}}\right)=\left(\zeta_{\mathcal{K}}^{\mathcal{T}_{\alpha}}\left(u_{\mathcal{T}_{\alpha}}\right)\right)_{\underline{\mathcal{K}} \in \mathcal{T}_{\alpha}^{P}} \quad \text { with } \quad \zeta_{\mathcal{K}}^{\mathcal{T}_{\alpha}}\left(u_{\mathcal{T}_{\alpha}}\right)=u_{\mathcal{K}} .
$$

Noting $\underline{\sigma}$ the edges of the mesh $\mathcal{T}_{\alpha}^{P}, m_{\underline{\sigma}}$ their length and $d_{\mathcal{K}, \mathcal{L}}$ the distance between the centers $x_{\mathcal{K}}$ and $x_{\mathcal{L}}$ of two neighboring control volumes $\underline{\mathcal{K}}$ and $\underline{\mathcal{L}}$. Then, the discrete $H^{1}$ norm associated with the mesh $\mathcal{T}_{\alpha}^{P}$ is defined by,

$$
\left\|\zeta^{\mathcal{T}_{\alpha}}\left(u_{\tau_{\alpha}}\right)\right\|_{1, \tau_{\alpha}^{P}}^{2}=\sum_{\sigma \in \mathcal{E} \cup \mathcal{E}_{i n t}^{\alpha}} \frac{m_{\underline{\sigma}}}{d_{\mathcal{K}, \mathcal{L}}}\left|u_{\mathcal{K}}-u_{\mathcal{L}}\right|^{2} .
$$

Proposition 4.19. There exists $C_{8}>0$ independent of $\operatorname{size}(\mathcal{T})$ such that for any $u_{\mathcal{T}} \in \mathbb{R}^{\mathcal{T}}$ the following inequality holds,

$$
\left\|\zeta^{\mathcal{T}_{\alpha}}\left(\Psi_{\mathcal{T}}^{\Omega_{\alpha}}\left(u_{\mathcal{T}}\right)\right)\right\|_{1, \mathcal{T}_{\alpha}^{P}}^{2} \leq C_{\S}\left(\left|u_{\mathcal{T}}\right|_{1, \mathcal{T}}^{2}+\left\|u_{\partial \mathfrak{M}}\right\|_{1, \partial \mathfrak{M}}^{2}\right) .
$$

Proof. Using Definitions 4.17 and 4.18 of operators $\Psi_{\mathcal{T}}^{\Omega_{\alpha}}$ and $\zeta^{\tau_{\alpha}}$, we have

$$
\begin{aligned}
\left\|\zeta^{\tau_{\alpha}}\left(\Psi_{\mathcal{T}}^{\Omega_{\alpha}}\left(u_{\mathcal{T}}\right)\right)\right\|_{1, \tau_{\alpha}^{P}}^{2}= & \sum_{\sigma=\mathcal{K} \mid \mathcal{L} \in \mathcal{E}_{i n t}} \frac{m_{\sigma}}{d_{\mathcal{K}, \mathcal{L}}}\left(u_{\mathcal{K}}-u_{\mathcal{L}}\right)^{2}+\sum_{\sigma=\mathcal{L} \in \mathcal{E}_{\text {ext }}} \frac{m_{\mathbf{e}_{\mathcal{L}}}}{d\left(x_{\mathcal{K}}, x_{\mathcal{R}_{\mathcal{L}}^{1}}\right)}\left(u_{\mathcal{K}}-u_{\mathcal{R}_{\mathcal{L}}^{1}}\right)^{2} \\
& +\sum_{\sigma \in \mathcal{E}_{\alpha_{\alpha}}} \frac{m_{\mathbf{e}_{\mathcal{L}}^{i, i+1}}}{d\left(x_{\mathcal{R}_{\mathcal{L}}^{i}}, x_{\mathcal{R}_{\mathcal{L}}^{i+1}}\right)}\left(u_{\mathcal{R}_{\mathcal{L}}^{i}}-u_{\mathcal{R}_{\mathcal{L}}^{i+1}}\right)^{2} \\
& +\sum_{\sigma \in \mathcal{E}_{\alpha_{\perp}}} \frac{m_{\sigma}}{d\left(x_{\mathcal{R}_{\mathcal{L}}^{i}}, x_{\left.\mathcal{R}_{\mathcal{L}^{\prime}}^{i}\right)}\right.}\left(u_{\mathcal{R}_{\mathcal{L}}^{i}}-u_{\mathcal{R}_{\mathcal{L}^{\prime}}^{i}}\right)^{2} .
\end{aligned}
$$

Now we have to express the last three sums in the right hand side of this term in function of $u_{\mathcal{T}}$. The definitions of $u_{\mathcal{R}_{\mathcal{L}}^{1}}$ and $\theta$ given in Definition 4.17 lead up to,

$$
\left|u_{\mathcal{L}}-u_{\mathcal{R}_{\mathcal{L}}^{1}}\right|=\left|u_{\mathcal{L}}\right|\left|\theta\left(d\left(x_{\mathcal{L}}, \Gamma\right)\right)-\theta\left(d\left(x_{\mathcal{R}_{\mathcal{L}}^{1}}, \Gamma\right)\right)\right| \leq C_{\theta} \frac{h_{\alpha}}{2}\left|u_{\mathcal{L}}\right|,
$$


then noting that $\frac{h_{\alpha}}{2} \leq d\left(x_{\mathcal{K}}, x_{\mathcal{R}_{\mathcal{L}}^{1}}\right)$ and $d\left(x_{\mathcal{K}}, x_{\mathcal{L}}\right) \leq d\left(x_{\mathcal{K}}, x_{\mathcal{R}_{\mathcal{L}}^{1}}\right)$ we have,

$$
\sum_{\sigma=\mathcal{L} \in \mathcal{E}_{e x t}} m_{\mathbf{e}_{\mathcal{L}}} \frac{\left|u_{\mathcal{K}}-u_{\mathcal{R}_{\mathcal{L}}^{1}}\right|^{2}}{d\left(x_{\mathcal{K}}, x_{\mathcal{R}_{\mathcal{L}}^{1}}\right)} \leq C_{\Gamma} \sum_{\sigma=\mathcal{L} \in \mathcal{E}_{\text {ext }}} m_{\mathbf{e}_{\mathcal{L}}} \frac{\left|u_{\mathcal{K}}-u_{\mathcal{L}}\right|^{2}}{d_{\mathcal{K}, \mathcal{L}}}+C_{\theta}^{2} h_{\alpha}\left\|u_{\partial \mathfrak{M}}\right\|_{0, \partial \mathfrak{M}}^{2} .
$$

In the same way, using the definitions of the centers $x_{\mathcal{R}_{\mathcal{L}}^{i}}$ et $x_{\mathcal{R}_{\mathcal{L}}^{i+1}}$ and noting that $d\left(x_{\mathcal{R}_{\mathcal{L}}^{i}}, x_{\mathcal{R}_{\mathcal{L}}^{i+1}}\right)=$ $h_{\alpha}$ there exists $C_{\Gamma, \alpha, \kappa}>0$ independent of $\operatorname{size}(\mathcal{T})$ such that,

$$
\sum_{\sigma \in \mathcal{E}_{\alpha_{\|}}} m_{\mathbf{e}_{\mathcal{L}}^{i, i+1}} \frac{\left|u_{\mathcal{R}_{\mathcal{L}}^{i}}-u_{\mathcal{R}_{\mathcal{L}}^{i+1}}\right|^{2}}{d\left(x_{\mathcal{R}_{\mathcal{L}}^{i+1}}, x_{\mathcal{R}_{\mathcal{L}}^{i+1}}\right)} \leq C_{\theta}^{2} C_{\Gamma, \alpha, \kappa} h_{\alpha} \sum_{\sigma \in \mathcal{E}_{\alpha_{\|}}} m_{\mathbf{e}_{\mathcal{L}}}\left|u_{\mathcal{L}}\right|^{2}
$$

We can remark that, at each boundary control volume $\mathcal{L} \in \partial \mathfrak{M}$ corresponds $n_{\alpha}-1$ (with $n_{\alpha}=\frac{\alpha}{h_{\alpha}}$ ) edges $\sigma \in \mathcal{E}_{\alpha_{\|}}$so we obtain

$$
\sum_{\sigma \in \mathcal{E}_{\alpha_{\|}}} m_{\mathbf{e}_{\mathcal{L}}^{i, i+1}} \frac{\left|u_{\mathcal{R}_{\mathcal{L}}^{i}}-u_{\mathcal{R}_{\mathcal{L}}^{i+1}}\right|^{2}}{d\left(x_{\mathcal{R}_{\mathcal{L}}^{i+1}}, x_{\mathcal{R}_{\mathcal{L}}^{i+1}}\right)} \leq \alpha C_{\theta}^{2} C_{\Gamma, \alpha, \kappa}\left\|u_{\partial \mathfrak{M}}\right\|_{0, \partial \mathfrak{M}}^{2} .
$$

Finally, thanks to the definitions of $u_{\mathcal{R}_{\mathcal{L}}^{i}}$ and $u_{\mathcal{R}^{i}}$, and since $\theta \leq 1$, by noting that for any $\sigma \in \mathcal{E}_{\alpha_{\perp}}, m_{\sigma}=h_{\alpha}$ there exists $C_{\Gamma, \alpha, \kappa}>0$ independent of $\operatorname{size}(\mathcal{T})$ such that,

$$
\sum_{\sigma \in \mathcal{E}_{\alpha_{\perp}}} \frac{m_{\sigma}}{d\left(x_{\mathcal{R}_{\mathcal{L}}^{i}}, x_{\mathcal{R}_{\mathcal{L}^{\prime}}^{i}}\right)}\left(u_{\mathcal{R}_{\mathcal{L}}^{i}}-u_{\mathcal{R}_{\mathcal{L}^{\prime}}^{i}}\right)^{2} \leq C_{\Gamma, \alpha, \kappa} h_{\alpha} \sum_{\sigma \in \mathcal{E}_{\alpha_{\perp}}} \frac{\left|u_{\mathcal{L}}-u_{\mathcal{L}^{\prime}}\right|^{2}}{d_{\mathcal{L}, \mathcal{L}^{\prime}}} .
$$

At each vertex $\mathbf{v}=\mathcal{L} \mid \mathcal{L}^{\prime} \in \mathcal{V}$ we can associate $n_{\alpha}$ edges $\sigma \in \mathcal{E}_{\alpha_{\perp}}$, thus

$$
\sum_{\sigma \in \mathcal{E}_{\alpha_{\perp}}} \frac{m_{\sigma}}{d\left(x_{\mathcal{R}_{\mathcal{L}}^{i}}, x_{\mathcal{R}_{\mathcal{L}^{\prime}}}\right)}\left(u_{\mathcal{R}_{\mathcal{L}}^{i}}-u_{\mathcal{R}_{\mathcal{L}^{\prime}}^{i}}\right)^{2} \leq \alpha C_{\Gamma, \alpha, \kappa}\left|u_{\partial \mathfrak{M}}\right|_{1, \partial \mathfrak{M}}^{2}
$$

Combining estimates 4.17, 4.18) and 4.19) we can conclude the proof.

Now we can apply the classical lemma of space translate estimates (see for instance [10, Lemma 3.3]) to the function $\zeta^{\mathcal{T}_{\alpha}}\left(\Psi_{\mathcal{T}}^{\Omega_{\alpha}}\left(u_{\mathcal{T}}\right)\right) \in \mathbb{R}^{\mathcal{T}_{\alpha}^{P}}$ on the polygonal mesh $\mathcal{T}_{\alpha}^{P}$ (by noticing that $\left.\operatorname{size}\left(\mathcal{T}_{\alpha}^{P}\right) \leq C_{\alpha, \Gamma} \operatorname{size}(\mathcal{T})\right)$

For a function $u_{\mathcal{T}_{\alpha}} \in \mathbb{R}^{\tau_{\alpha}}$ (respectively $u_{\mathcal{T}_{\alpha}^{P}} \in \mathbb{R}^{\tau_{\alpha}^{P}}$ ), we denote $\widetilde{u_{\tau_{\alpha}}}$ (respectively $\widetilde{u_{\tau_{\alpha}^{P}}}$ ) the extension by 0 of $u_{\mathcal{T}_{\alpha}}$ (respectively $u_{\mathcal{T}_{\alpha}^{P}}$ ) outside of $\Omega_{\alpha}$ (respectively $\Omega_{\alpha}^{P}$ ).

THEOREM 4.20 (Estimation of space translates).

There exists $C_{9}>0$ independent of $\operatorname{size}(\mathcal{T})$ such that for any $u_{\mathcal{T}} \in \mathbb{R}^{\mathcal{T}}$ and for any $\eta \in \mathbb{R}^{2}$ the following estimate holds,

$$
\begin{aligned}
\| \widetilde{\zeta^{\tau_{\alpha}}}\left(\Psi_{\mathcal{T}}^{\Omega_{\alpha}}\left(u_{\mathcal{T}}\right)\right)(.+\eta)- & -\widetilde{\zeta^{\tau_{\alpha}}}\left(\Psi_{\mathcal{T}}^{\Omega_{\alpha}}\left(u_{\mathcal{T}}\right)\right) \|_{L^{2}\left(\mathbb{R}^{2}\right)}^{2} \\
& \leq C_{9} \mid(|\eta|+\operatorname{size}(\mathcal{T}))\left(\left|u_{\mathcal{T}}\right|_{1, \mathcal{T}}^{2}+\left\|u_{\partial \mathfrak{M}}\right\|_{1, \partial \mathfrak{M}}^{2}\right) .
\end{aligned}
$$

Let us remark that the estimate of space translates uses the extension operator $\widetilde{\zeta^{\mathcal{T}_{\alpha}}}\left(\Psi_{\mathcal{T}}^{\Omega_{\alpha}}\left(u_{\mathcal{T}}\right)\right)$ defined on the approximate polygonal domain $\Omega^{P}$. However, to prove the convergence theorem we would like to deal with the original mesh $\mathcal{T}$ of the original domain $\Omega$ and the extension operator $\Psi_{\mathcal{T}}^{\Omega_{\alpha}}\left(u_{\mathcal{T}}\right)$ defined on this domain. So we have to begin by proving that when the mesh size tends to 0 these operators converge towards the same limit. 
Proposition 4.21. There exists $C_{10}>0$ independent of $\operatorname{size}(\mathcal{T})$ such that for any function $u_{\mathcal{T}} \in \mathbb{R}^{\mathcal{T}}$ the following identity holds for a small enough $\operatorname{size}(\mathcal{T})$,

$$
\left\|\widetilde{\Psi_{\mathcal{T}}^{\Omega_{\alpha}}}\left(u_{\mathcal{T}}\right)-\widetilde{\zeta^{\mathcal{T}_{\alpha}}}\left(\Psi_{\mathcal{T}}^{\Omega_{\alpha}}\left(u_{\mathcal{T}}\right)\right)\right\|_{L^{2}\left(\mathbb{R}^{2}\right)}^{2} \leq C_{10} \operatorname{size}(\mathcal{T})^{2}\left(\left|u_{\mathcal{T}}\right|_{1, \mathcal{T}}^{2}+\left\|u_{\partial \mathfrak{M}}\right\|_{1, \partial \mathfrak{M}}^{2}\right) .
$$

Proof. For any control volume $\mathcal{K} \in \mathcal{T}_{\alpha}$, we note $1_{\mathcal{K}}$ the indicator function of the control volume $\mathcal{K}$ and $1_{\underline{\mathcal{K}}}$ those of the control volume $\underline{\mathcal{K}}$. Then, for a small enough $\operatorname{size}(\mathcal{T})$, for any $w_{\mathcal{T}_{\alpha}} \in \mathbb{R}^{\tau_{\alpha}}$ we have,

$$
\begin{aligned}
\left|\widetilde{w_{\mathcal{T}_{\alpha}}}-\widetilde{\mathcal{\zeta}^{\mathcal{T}_{\alpha}}}\left(w_{\mathcal{T}_{\alpha}}\right)\right|= & \left|\sum_{\mathcal{K} \in \mathcal{T}_{\alpha}} w_{\mathcal{K}} 1_{\mathcal{K}}-\sum_{\mathcal{K} \in \mathcal{T}_{\alpha}} w_{\mathcal{K}} 1_{\underline{\mathcal{K}}}\right| \\
= & \mid \sum_{\sigma \in \mathcal{E} \cup \mathcal{E}_{\text {int }}^{\alpha}}\left(1_{\mathcal{K} \cap \underline{\mathcal{L}}}\left(w_{\mathcal{K}}-w_{\mathcal{L}}\right)+1_{\mathcal{L} \cap \underline{\mathcal{K}}}\left(w_{\mathcal{L}}-w_{\mathcal{K}}\right)\right) \\
& +\sum_{\sigma \in \mathcal{E}_{\text {ext }}^{\alpha}} w_{\mathcal{K}}\left(1_{\mathcal{K}^{e x t}}-1_{\underline{\mathcal{K}}^{e x t}} \mid\right.
\end{aligned}
$$

where $\mathcal{K}^{e x t}=\left\{x \in\left(\Omega^{P} \cup \Omega_{\alpha}^{P}\right)^{c}: x \in \mathcal{K}\right.$ and $\left.x \notin \underline{\mathcal{K}}\right\}$ and $\underline{\mathcal{K}}^{e x t}=\left\{x \in\left(\Omega \cup \Omega_{\alpha}\right)^{c}: x \in\right.$ $\underline{\mathcal{K}}$ and $x \notin \mathcal{K}\}$.

The sets which appear in the indicator functions are pairwise disjoint thus we can write,

$\left|\widetilde{w_{\mathcal{T}_{\alpha}}}-\widetilde{\zeta^{\tau_{\alpha}}}\left(w_{\mathcal{T}_{\alpha}}\right)\right|^{2}=\sum_{\sigma \in \mathcal{E} \cup \mathcal{E}_{i n t}^{\alpha}}\left(1_{\mathcal{K} \cap \underline{\mathcal{L}}}-1_{\mathcal{L} \cap \underline{\mathcal{K}}}\right)^{2}\left|w_{\mathcal{K}}-w_{\mathcal{L}}\right|^{2}+\sum_{\sigma \in \mathcal{E}_{e x t}^{\alpha}}\left(1_{\mathcal{K}^{e x t}}-1_{\underline{\mathcal{K}}^{e x t}}\right)^{2}\left|w_{\mathcal{K}}\right|^{2}$.

Noting that for any $w_{\mathcal{T}_{\alpha}} \in \mathbb{R}^{\tau_{\alpha}}$,

$$
\left\|\widetilde{w_{\mathcal{T}_{\alpha}}}-\widetilde{\zeta^{\tau_{\alpha}}}\left(w_{\mathcal{T}_{\alpha}}\right)\right\|_{L^{2}\left(\mathbb{R}^{2}\right)}^{2}=\int_{\Omega \cup \Omega_{\alpha} \cup \Omega_{\alpha}^{P}}\left|\widetilde{w_{\mathcal{T}_{\alpha}}}(x)-\widetilde{\zeta^{\tau_{\alpha}}}\left(w_{\mathcal{T}_{\alpha}}\right)(x)\right|^{2} \mathrm{~d} x
$$

By noting that for any $\sigma=\mathcal{K} \mid \mathcal{L} \in \mathcal{E} \cap \mathcal{E}_{\text {int }}^{\alpha}$ (respectively $\sigma \in \mathcal{E}_{\text {ext }}^{\alpha}$ ) the set $\mathcal{K} \cup \underline{\mathcal{L}}$ and $\underline{\mathcal{K}} \cup \mathcal{L}$ (respectively $\mathcal{K}^{e x t}$ and $\underline{\mathcal{K}}^{e x t}$ ) are disjoint we have,

$$
\left\|\widetilde{w_{\mathcal{T}_{\alpha}}}-\widetilde{\zeta^{\tau_{\alpha}}}\left(w_{\mathcal{T}_{\alpha}}\right)\right\|_{L^{2}\left(\mathbb{R}^{2}\right)}^{2}=\sum_{\sigma \in \mathcal{E} \cup \mathcal{E}_{i n t}^{\alpha}}\left(m_{\mathcal{K} \cap \underline{\mathcal{L}}}+m_{\mathcal{L} \cap \underline{\mathcal{K}}}\right)\left|w_{\mathcal{K}}-w_{\mathcal{L}}\right|^{2}+\sum_{\sigma \in \mathcal{E}_{\text {ext }}^{\alpha}}\left(m_{\mathcal{K}^{e x t}}+m_{\underline{\mathcal{K}}^{e x t}}\right)\left|w_{\mathcal{K}}\right|^{2} .
$$

Thus there exists a constant $C_{\Omega_{\alpha}}(\operatorname{reg}(\mathcal{T}))>0$ independent of $\operatorname{size}(\mathcal{T})$ such that,

$$
\left|\widetilde{w_{\mathcal{T}_{\alpha}}}-\widetilde{\zeta^{\tau_{\alpha}}}\left(w_{\mathcal{T}_{\alpha}}\right)\right|^{2} \leq C_{\Omega_{\alpha}}(\operatorname{reg}(\mathcal{T})) \operatorname{size}(\mathcal{T})^{2}\left(\left\|\zeta^{\mathcal{T}_{\alpha}}\left(w_{\mathcal{T}_{\alpha}}\right)\right\|_{1, \mathcal{T}_{\alpha}^{P}}^{2}+\sum_{\sigma \in \mathcal{E}_{e x t}^{\alpha}} \operatorname{size}(\mathcal{T})\left|w_{\mathcal{K}}\right|^{2}\right) .
$$

Setting $w_{\mathcal{T}_{\alpha}}=\Psi_{\mathcal{T}}^{\Omega_{\alpha}}\left(u_{\mathcal{T}}\right)$ and noticing that if $\mathcal{K} \in \mathcal{T}_{\alpha}^{P}$ such that $\sigma=\psi(\mathcal{L} \times\{\alpha\}) \in \mathcal{E}_{\text {ext }}^{\alpha} \cap \mathcal{E}_{\mathcal{K}}$, we have

$$
\left|w_{\mathcal{K}}\right|=\left|\Psi_{\mathcal{K}}^{\Omega_{\alpha}}\left(u_{\mathcal{T}}\right)\right|=\left|u_{\mathcal{L}} \theta\left(d\left(x_{\mathcal{R}_{\mathcal{L}}^{n_{\alpha}}}, \Gamma\right)\right)\right| \leq\left|u_{\mathcal{L}}\right|
$$

we obtain,

$$
\left|\widetilde{\Psi_{\mathcal{T}}^{\Omega_{\alpha}}}\left(u_{\mathcal{T}}\right)-\widetilde{\zeta^{\tau_{\alpha}}}\left(\Psi_{\mathcal{T}}^{\Omega_{\alpha}}\left(u_{\mathcal{T}}\right)\right)\right|^{2} \leq C_{\Omega_{\alpha}}(\operatorname{reg}(\mathcal{T})) \operatorname{size}(\mathcal{T})^{2}\left(\left\|\zeta^{\mathcal{T}_{\alpha}}\left(\Psi_{\mathcal{T}}^{\Omega_{\alpha}}\left(u_{\mathcal{T}}\right)\right)\right\|_{1, \mathcal{T}_{\alpha}^{P}}^{2}+\left\|u_{\partial \mathfrak{M}}\right\|_{0, \partial \mathfrak{M}}^{2}\right)
$$


and Proposition 4.19 conclude the proof.

PROPOSITION 4.22. Let $\left(u_{\mathcal{T}^{(m)}}\right)_{m \in \mathbb{N}}$ be a sequence of discrete functions in $\mathbb{R}^{\mathcal{T}^{(m)}}$ associated with a sequence of discretizations such that the mesh size $\operatorname{size}\left(\mathcal{T}^{(m)}\right)$ tends to 0 . We assume that the regularity $\operatorname{reg}\left(\mathcal{T}^{(m)}\right)$ is bounded and that there exists $M>0$ independent of $\operatorname{size}\left(\mathcal{T}^{(m)}\right)$ satisfying

$$
\left\|u_{\mathcal{T}^{(m)}}\right\|_{1, \mathcal{T}^{(m)}} \leq M \quad \text { and } \quad\left\|u_{\partial \mathfrak{M}(m)}\right\|_{1, \partial \mathfrak{M}(m)} \leq M, \quad \forall m \geq 0 .
$$

Then, we can extract a subsequence, still referred to as $\left(u_{\left.\mathcal{T}^{(m)}\right)}\right)_{m \in \mathbb{N}}$ for simplicity, which is strongly converging in $L^{2}(\Omega)$ towards a certain function $u \in H^{1}(\Omega)$ whose trace belongs to $H^{1}(\Gamma)$ and such that $\left(u_{\partial \mathfrak{m}}(m)\right)_{m \in \mathbb{N}}$ is strongly converging in $L^{2}(\Gamma)$ towards $u_{1 \Gamma}$. Furthermore up to a subsequence, $\left(\nabla^{\mathcal{T}} u_{\mathcal{T}(m)}\right)_{m \in \mathbb{N}}$ weakly converges towards $\nabla u$ in $L^{2}(\Omega)$ and $\left(\nabla_{\Gamma}^{\partial \mathfrak{M}} u_{\partial \mathfrak{M}(m)}\right)_{m \in \mathbb{N}}$ weakly converges towards $\nabla_{\Gamma} u_{1 \Gamma}$ in $L^{2}(\Gamma)$.

In our study, the functions are also time-dependent thus we give below an adapted version for these functions.

PROPOSITION 4.23. Let $\left(\left(u_{\mathcal{T}}^{\Delta t}\right)^{(m)}\right)_{m \in \mathbb{N}}$ be a sequence of functions associated with a sequence of discretizations such that the time step and the mesh size, $\Delta t^{(m)}$ and $\operatorname{size}\left(\mathcal{T}^{(m)}\right)$ respectively, tend to 0 and such that the regularity $\operatorname{reg}\left(\mathcal{T}^{(m)}\right)$ is bounded. We assume that there exists a positive constant $M$ such that,

$$
\sup _{n \leq N^{(m)}}\left\|\left(u_{\mathcal{T}^{n}}^{n}\right)^{(m)}\right\|_{1, \mathcal{T}^{(m)}} \leq M \text { and } \sup _{n \leq N^{(m)}}\left\|\left(u_{\partial \mathfrak{M}}^{n}\right)^{(m)}\right\|_{1, \partial \mathfrak{M}(m)} \leq M, \quad \forall m \geq 0 .
$$

Moreover, we assume that there exists a function $\widetilde{u} \in L^{2}\left((0, T) \times \mathbb{R}^{2}\right)$ satisfying (up to a subsequence) the following convergence,

$$
\widetilde{\zeta^{\mathcal{T}_{\alpha}}}\left(\Psi_{\mathcal{T}}^{\Omega_{\alpha}}\left(\left(u_{\mathcal{T}}^{\Delta t}\right)^{(m)}\right)\right) \underset{m \rightarrow+\infty}{\longrightarrow} \widetilde{u} \text { in } L^{2}\left((0, T) \times \mathbb{R}^{2}\right) .
$$

We denote by $u$ the restriction of $\widetilde{u}$ to $\Omega$, then $u \in L^{2}\left(0, T ; H^{1}(\Omega)\right)$ and $u_{1 \Gamma} \in L^{2}\left(0, T ; H^{1}(\Gamma)\right)$. Furthermore the following convergences hold (up to a subsequence),

$$
\begin{gathered}
\left(u_{\mathcal{\tau}}^{\Delta t}\right)^{(m)} \underset{m \rightarrow+\infty}{\longrightarrow} u \text { in } L^{2}((0, T) \times \Omega), \quad\left(u_{\partial \mathfrak{M}}^{\Delta t}\right)^{(m)} \underset{m \rightarrow+\infty}{\longrightarrow} u_{1 \Gamma} \text { in } L^{2}((0, T) \times \Gamma), \\
\left(\nabla^{\mathcal{\tau}} u_{\mathcal{T}}^{\Delta t}\right)^{(m)} \underset{m \rightarrow+\infty}{\longrightarrow} \nabla u \text { weakly in } L^{2}((0, T) \times \Omega), \\
\left(\nabla_{\Gamma}^{\partial \mathfrak{M}} u_{\partial \mathfrak{M}}^{\Delta t}\right)^{(m)} \underset{m \rightarrow+\infty}{\longrightarrow} \nabla_{\Gamma} u_{\mid \Gamma} \text { weakly in } L^{2}((0, T) \times \Gamma) .
\end{gathered}
$$

Proof of Proposition 4.22 We split this proof into several steps and we notice that all the convergences below occur up to a subsequence.

- We prove that the sequence $\left(\Psi_{\mathcal{T}}^{\Omega_{\alpha}}\left(u_{\left.\mathcal{T}^{(m)}\right)}\right)\right)_{m \in \mathbb{N}}$ strongly converges in $L^{2}\left(\mathbb{R}^{2}\right)$ towards a certain $\widetilde{u} \in H^{1}\left(\mathbb{R}^{2}\right)$.

Theorem 4.20 and assumption 4.20 imply that for all $m \in \mathbb{N}$,

$$
\begin{aligned}
\| \widetilde{\zeta^{\mathcal{T}_{\alpha}}}\left(\Psi_{\mathcal{T}}^{\Omega_{\alpha}}\left(u_{\mathcal{T}^{(m)}}\right)\right)(.+\eta)-\widetilde{\zeta^{\mathcal{T}_{\alpha}}}\left(\Psi_{\mathcal{T}}^{\Omega_{\alpha}}\left(u_{\left.\mathcal{T}^{(m)}\right)}\right) \|_{L^{2}\left(\mathbb{R}^{2}\right)}^{2}\right. \\
\leq 2 M^{2} C_{9} \eta \mid(|\eta|+\operatorname{size}(\mathcal{T})) .
\end{aligned}
$$

Moreover Proposition 4.21 gives,

$$
\begin{aligned}
\left\|\widetilde{\zeta^{\tau_{\alpha}}}\left(\Psi_{\mathcal{T}}^{\Omega_{\alpha}}\left(u_{\mathcal{T}^{(m)}}\right)\right)\right\|_{L^{2}\left(\mathbb{R}^{2}\right)}^{2} \leq & 2 C_{\overline{10}} \operatorname{size}\left(\mathcal{T}^{(m)}\right)^{2}\left(\left|u_{\mathcal{T}^{(m)}}\right|_{1, \mathcal{T}}^{2}+\left\|u_{\partial \mathfrak{M}(m)}\right\|_{1, \partial \mathfrak{M}}^{2}\right) \\
& +2 \| \widetilde{\Psi_{\mathcal{T}}^{\Omega_{\alpha}}}\left(u_{\left.\mathcal{T}^{(m)}\right)} \|_{L^{2}\left(\mathbb{R}^{2}\right)}^{2} .\right.
\end{aligned}
$$


Furthermore, using Definition 4.17 of the operator $\Psi_{\mathcal{T}}^{\Omega_{\alpha}}$,

$$
\left\|\widetilde{\Psi_{\mathcal{T}}^{\Omega_{\alpha}}}\left(u_{\left.\mathcal{T}^{(m)}\right)}\right)\right\|_{L^{2}\left(\mathbb{R}^{2}\right)}^{2} \leq M_{\Omega}\left\|u_{\mathcal{T}^{(m)}}\right\|_{0, \mathfrak{M}(m)}^{2}+n_{\alpha} C_{\alpha, \kappa}\left\|u_{\partial \mathfrak{M}(m)}\right\|_{0, \partial \mathfrak{M}(m)}^{2} ;
$$

and thus assumption 4.20 ) gives that $\left.\widetilde{\zeta^{\tau_{\alpha}}}\left(\Psi_{\mathcal{T}}^{\Omega_{\alpha}}\left(u_{\mathcal{T}^{(m)}}\right)\right)\right)_{m \in \mathbb{N}}$ is bounded in $L^{2}\left(\mathbb{R}^{2}\right)$.

Then, thanks to the Kolmogorov theorem we can extract a subsequence, still referred to as $\left.\widetilde{\zeta^{\tau_{\alpha}}}\left(\Psi_{\mathcal{T}}^{\Omega_{\alpha}}\left(u_{\mathcal{T}^{(m)}}\right)\right)\right)_{m \in \mathbb{N}}$ for simplicity, and find $\widetilde{u} \in L^{2}\left(\mathbb{R}^{2}\right)$ such that

$$
\widetilde{\zeta^{\mathcal{T}_{\alpha}}}\left(\Psi_{\mathcal{T}}^{\Omega_{\alpha}}\left(u_{\mathcal{T}^{(m)}}\right)\right) \underset{m \rightarrow+\infty}{\longrightarrow} \widetilde{u} \text { in } L^{2}\left(\mathbb{R}^{2}\right)
$$

Passing to the limit in equation 4.23, convergence (4.24) gives that $\widetilde{u} \in H^{1}\left(\mathbb{R}^{2}\right)$.

- According to Proposition 4.21 and assumption 4.20 we have,

$$
\left|\widetilde{\zeta^{\mathcal{T}_{\alpha}}}\left(\Psi_{\mathcal{T}}^{\Omega_{\alpha}}\left(u_{\mathcal{T}^{(m)}}\right)\right)-\widetilde{\Psi_{\mathcal{T}}^{\Omega_{\alpha}}}\left(u_{\mathcal{T}^{(m)}}\right)\right| \underset{m \rightarrow+\infty}{\longrightarrow} 0 \text { in } L^{2}\left(\mathbb{R}^{2}\right),
$$

thus setting $u=\widetilde{u}_{\mid \Omega}$ we obtain, by restriction to $\Omega$,

$$
u_{\mathcal{T}^{(m)}} \underset{m \rightarrow+\infty}{\longrightarrow} u \text { in } L^{2}(\Omega) \quad \text { and } \quad u \in H^{1}(\Omega) .
$$

- Let us prove that the subsequence $\left(u_{\partial \mathfrak{M}}(m)\right)_{m \in \mathbb{N}}$ strongly converges in $L^{2}(\Gamma)$ towards $u_{1 \Gamma}=\operatorname{Tr}(u)$.

Let $\Omega_{\alpha / 2}=\left\{x \in \Omega^{c}, d(x, \Gamma)<\frac{\alpha}{2}\right\}$ then Definition 4.17 of $\Psi_{\mathcal{T}}^{\Omega_{\alpha}}$ and especially the fact that $\theta \equiv 1$ on $\left[0, \frac{\alpha}{2}\right]$ implies,

$$
\left(\Psi_{\mathcal{T}}^{\Omega_{\alpha}}\left(u_{\mathcal{T}^{(m)}}\right)\right)(x)=u_{\partial \mathfrak{M}(m)}\left(\mathbb{P}_{\Gamma} x\right), \quad \forall x \in \Omega_{\alpha / 2} .
$$

Hence for any $x_{\Gamma} \in \Gamma, s \in\left[0, \frac{\alpha}{2}\right]$,

$$
u_{\partial \mathfrak{M}(m)}\left(x_{\Gamma}\right)=\left(\Psi_{\mathcal{T}}^{\Omega_{\alpha}}\left(u_{\mathcal{T}^{(m)}}\right)\right)\left(x_{\Gamma}+s \overrightarrow{\mathbf{n}}\left(x_{\Gamma}\right)\right)
$$

and for any $x_{\Gamma} \in \Gamma$ we thus have,

$$
u_{\partial \mathfrak{M}(m)}\left(x_{\Gamma}\right)=\frac{2}{\alpha} \int_{0}^{\frac{\alpha}{2}}\left(\Psi_{\mathcal{T}}^{\Omega_{\alpha}}\left(u_{\mathcal{T}^{(m)}}\right)\right)\left(x_{\Gamma}+s \overrightarrow{\mathbf{n}}\left(x_{\Gamma}\right)\right) \mathrm{d} s .
$$

For any $x_{\Gamma} \in \Gamma$ we define,

$$
w\left(x_{\Gamma}\right)=\frac{2}{\alpha} \int_{0}^{\frac{\alpha}{2}} \widetilde{u}\left(x_{\Gamma}+s \overrightarrow{\mathbf{n}}\left(x_{\Gamma}\right)\right) \mathrm{d} s .
$$

By the change of variables $x=x_{\Gamma}+s \overrightarrow{\mathbf{n}}\left(x_{\Gamma}\right)$ on $\Omega_{\alpha / 2}$ we obtain,

$$
\int_{\Gamma}\left|w\left(x_{\Gamma}\right)-u_{\partial \mathfrak{M}(m)}\left(x_{\Gamma}\right)\right|^{2} \mathrm{~d} x_{\Gamma} \leq C_{\alpha, \kappa}\left\|\widetilde{u}-\Psi_{\mathcal{T}}^{\Omega_{\alpha}}\left(u_{\mathcal{T}^{(m)}}\right)\right\|_{L^{2}\left(\Omega_{\alpha / 2}\right)}^{2} .
$$

Thanks to convergences 4.24 and 4.25) we have $\Psi_{\mathcal{T}}^{\Omega_{\alpha}}\left(u_{\mathcal{T}^{(m)}}\right) \underset{m \rightarrow+\infty}{\longrightarrow} \widetilde{u}$ in $L^{2}\left(\mathbb{R}^{2}\right)$, thus we can deduce

$$
u_{\partial \mathfrak{M}(m)} \underset{m \rightarrow+\infty}{\longrightarrow} w=\frac{2}{\alpha} \int_{0}^{\frac{\alpha}{2}} \widetilde{u}(.+s \overrightarrow{\mathbf{n}}(.)) \mathrm{d} s \text { in } L^{2}(\Gamma) .
$$


Using the change of variables $x=x_{\Gamma}+s \overrightarrow{\mathbf{n}}\left(x_{\Gamma}\right)$ on $\Omega_{\alpha / 2}$ and then equality 4.26) together with convergence (4.27) we obtain,

$$
\int_{\Omega_{\alpha / 2}}\left|\left(\Psi_{\mathcal{T}}^{\Omega_{\alpha}}\left(u_{\left.\mathcal{T}^{(m)}\right)}\right)\right)(x)-w\left(\mathbb{P}_{\Gamma} x\right)\right|^{2} \underset{m \rightarrow+\infty}{\longrightarrow} 0 .
$$

Finally, recalling that $\Psi_{\mathcal{T}}^{\Omega_{\alpha}}\left(u_{\mathcal{T}^{(m)}}\right) \underset{m \rightarrow+\infty}{\longrightarrow} \widetilde{u}$ in $L^{2}\left(\mathbb{R}^{2}\right)$ we deduce

$$
w\left(\mathbb{P}_{\Gamma} x\right)=\widetilde{u}(x), \quad \forall x \in \Omega_{\alpha / 2} .
$$

Therefore, the function $w$ is the trace of $\widetilde{u}$ on $\partial\left(\Omega^{c}\right)=\partial \Omega$ and $\widetilde{u} \in H^{1}\left(\mathbb{R}^{2}\right)$ thereby it is also the trace of $u$. Thus convergence (4.27) implies

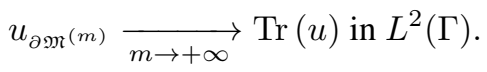

Now we have to verify that $u_{I \Gamma}=\operatorname{Tr}(u) \in H^{1}(\Gamma)$. Thanks to equality (4.28) we can observe that for any $x \in \Omega_{\alpha / 2}, \widetilde{u}(x)$ does not depend on $d(x, \Gamma)$, then we have

$$
\nabla \widetilde{u}(x)=\nabla_{\Gamma_{d(x, \Gamma)}} \widetilde{u}(x), \quad \forall x \in \Omega_{\alpha / 2} .
$$

We can deduce that for any $x \in \Omega_{\alpha / 2}$ :

$$
\nabla \widetilde{u}(x)=\frac{1}{\left(1-d(x, \Gamma) \kappa\left(\mathbb{P}_{\Gamma} x\right)\right)} \nabla_{\Gamma} w\left(\mathbb{P}_{\Gamma} x\right),
$$

namely for any $x_{\Gamma} \in \Gamma$ and for any $s \in\left[0, \frac{\alpha}{2}\right]$ we can write,

$$
\nabla_{\Gamma} u_{1 \Gamma}\left(x_{\Gamma}\right)=\left(1-s \kappa\left(x_{\Gamma}\right)\right) \nabla \widetilde{u}\left(x_{\Gamma}+s \overrightarrow{\mathbf{n}}\left(x_{\Gamma}\right)\right) .
$$

Integrating this equation for $s \in\left[0, \frac{\alpha}{2}\right]$ and for any $x_{\Gamma} \in \Gamma$, then using the change of variables $x=x_{\Gamma}+s \overrightarrow{\mathbf{n}}\left(x_{\Gamma}\right)$ on $\Omega_{\alpha / 2}$ we obtain,

$$
\int_{\Gamma}\left|\nabla_{\Gamma} u_{1 \Gamma}\left(x_{\Gamma}\right)\right|^{2} \mathrm{~d} x_{\Gamma} \leq \frac{2}{\alpha}\left(1+\frac{\alpha}{2}\|\kappa\|_{\infty}\right) \int_{\Omega_{\alpha / 2}}|\nabla \widetilde{u}(x)|^{2} \mathrm{~d} x .
$$

Since $\widetilde{u} \in H^{1}\left(\mathbb{R}^{2}\right)$ we conclude that $\operatorname{Tr}(u) \in H^{1}(\Gamma)$.

- Since $\left(u_{\mathcal{T}^{(m)}}\right)_{m \in \mathbb{N}}$ converges in $L^{2}(\Omega)$ towards $u \in H^{1}(\Omega)$, Proposition 4.13 together with the $H^{1}(\Omega)$-bound 4.20 gives that $\left(\nabla u_{\mathcal{T}^{(m)}}\right)_{m \in \mathbb{N}}$ weakly converges in $L^{2}(\Omega)$ towards $\nabla u$.

- Let us prove that the subsequence $\left(\nabla_{\Gamma}^{\partial \mathfrak{M}} u_{\partial \mathfrak{M}(m)}\right)_{m \in \mathbb{N}}$ weakly converges in $L^{2}(\Gamma)$ towards $\nabla_{\Gamma} u_{1 \Gamma}$.

We consider a vector field $\phi: \Gamma \rightarrow \mathbb{R}^{2}$ of $\mathcal{C}^{\infty}$ class and tangent to the hypersurface $\Gamma$. The sequence $\left(u_{\partial \mathfrak{m}(m)}\right)_{m \in \mathbb{N}}$ converges in $L^{2}(\Gamma)$ towards $u_{\iota \Gamma} \in H^{1}(\Gamma)$, thus by noting $\operatorname{div}_{\Gamma}$ the tangential divergence operator we have,

$$
\int_{\Gamma} u_{\partial \mathfrak{M}(m)}(x) \operatorname{div}_{\Gamma} \phi(x) \mathrm{d} \sigma(x) \underset{m \rightarrow+\infty}{\longrightarrow}-\int_{\Gamma} \nabla_{\Gamma} u_{\Gamma \Gamma}(x) \cdot \phi(x) \mathrm{d} \sigma(x) .
$$

Furthermore, thanks to the Stokes formula

$$
\begin{aligned}
\int_{\Gamma} u_{\partial \mathfrak{M}(m)}(x) \operatorname{div}_{\Gamma} \phi(x) \mathrm{d} \sigma(x)= & -\int_{\Gamma} \nabla_{\Gamma}^{\partial \mathfrak{M}} u_{\partial \mathfrak{M}(m)} \phi(x) \cdot \overrightarrow{\boldsymbol{\tau}}(x) \mathrm{d} \sigma(x) \\
& +\sum_{\mathbf{v}=\mathcal{L} \mid \mathcal{L}^{\prime} \in \mathcal{V}} d_{\mathcal{L}, \mathcal{L}^{\prime}} \nabla_{\Gamma, \mathbf{v}}^{\partial \mathfrak{M}} u_{\partial \mathfrak{M}(m)} R_{\mathbf{v}}^{\phi}
\end{aligned}
$$


where

$$
R_{\mathrm{v}}^{\phi}=\frac{1}{d_{\mathcal{L}, \mathcal{L}^{\prime}}} \int_{\gamma_{\mathcal{L} \mathcal{L}^{\prime}}} \phi(x) \cdot \overrightarrow{\boldsymbol{\tau}}(x) \mathrm{d} \sigma(x)-\phi(\mathbf{v}) \cdot \overrightarrow{\boldsymbol{\tau}}(\mathbf{v}) .
$$

Moreover, we have $m_{\gamma_{\mathcal{L} \mathcal{L}^{\prime}}}-d_{\mathcal{L}, \mathcal{L}^{\prime}}=\mathcal{O}\left(m_{\gamma_{\mathcal{L} \mathcal{L}^{\prime}}}\left(m_{\mathcal{L}}+m_{\mathcal{L}^{\prime}}\right)\right)$, then since $\phi$ is $\mathcal{C}^{\infty}$,

$$
R_{\mathrm{v}}^{\phi} \leq C\|\nabla \phi\|_{L^{\infty}(\Gamma)} \operatorname{size}(\mathcal{T}), \quad \forall \mathbf{v} \in \mathcal{V} .
$$

Furthermore, $H^{1}(\Gamma)$-bound (4.20) gives that $\left(\nabla_{\Gamma}^{\partial \mathfrak{M}} u_{\partial \mathfrak{M}(m)}\right)_{m \in \mathbb{N}}$ is bounded in $L^{2}(\Gamma)$ so

$$
\int_{\Gamma} \nabla_{\Gamma}^{\partial \mathfrak{M}} u_{\partial \mathfrak{M}(m)}(x) \phi(x) \cdot \overrightarrow{\boldsymbol{\tau}}(x) \mathrm{d} \sigma(x)=-\int_{\Gamma} u_{\partial \mathfrak{M}(m)}(x) \operatorname{div}_{\Gamma} \phi(x)+\mathcal{O}_{\phi}(\operatorname{size}(\mathcal{T})),
$$

and thanks to equation 4.29),

$$
\int_{\Gamma} \phi(x) \cdot \nabla_{\Gamma}^{\partial \mathfrak{M}} u_{\partial \mathfrak{M}(m)}(x) \overrightarrow{\boldsymbol{\tau}}(x) \mathrm{d} x \underset{m \rightarrow+\infty}{\longrightarrow} \int_{\Gamma} \phi(x) \cdot \nabla_{\Gamma} u_{l \Gamma}(x) \mathrm{d} x .
$$

Let us remark that in Proposition 4.23 we have to add assumption 4.22 which gives the $L^{2}$ strong convergence of a subsequence $\left(\left(u_{\mathcal{T}}^{\Delta t}\right)^{(m)}\right)_{m \in \mathbb{N}}$. Indeed, we consider time-dependent function so we have to add an estimate of time translates (that we are going to do in the sequel) to obtain this convergence. Thus, assumption (4.22) allows to obtain convergence (4.24).

We have given above the proof of Proposition 4.22 where the functions are supposed to be time-independent but we can remark that once the convergence (4.24) is obtained the sequel of the proof is almost the same.

DEFINITION 4.24 (Extension operator for time-dependent functions).

We define the extension operator $\zeta_{T}^{\mathcal{T}_{\alpha}}$ for time-dependent functions using operators $\Psi_{\mathcal{T}}^{\Omega_{\alpha}}$ and $\zeta^{T_{\alpha}}$ introduced in Definitions 4.17 and 4.18 as follows,

$$
\zeta_{T}^{\mathcal{T}_{\alpha}}\left(u_{\mathcal{T}}^{\Delta t}\right)(t, x)=\left\{\begin{array}{cl}
\widetilde{\zeta^{\tau_{\alpha}}}\left(\Psi_{\mathcal{T}}^{\Omega_{\alpha}}\left(u_{\mathcal{T}}^{\Delta t}\right)\right)(t, .) & \text { if } t \in[0, T] \\
0 & \text { otherwise }
\end{array}\right.
$$

By the sake of simplicity we use the notation $u_{\mathcal{T}_{\alpha}^{P}}^{\Delta t}(t,)=.\widetilde{\mathcal{\zeta}^{\mathcal{T}_{\alpha}}}\left(\Psi_{\mathcal{T}}^{\Omega_{\alpha}}\left(u_{\mathcal{T}}^{\Delta t}\right)\right)(t,$.$) .$

Lemma 4.25 ([14], Lemma A.1]). Let $\left(t^{n}\right)_{0 \leq n \leq N}$ such that $t^{0}=0, t^{n}=n \Delta t, t^{N}=T$, $\tau$ is a positive real number and $\chi_{\tau}^{n}: \mathbb{R} \rightarrow \mathbb{R}$ is the function defined as follows

$$
\chi_{\tau}^{n}(t)= \begin{cases}1 & \text { if } t<t^{n} \leq t+\tau \\ 0 & \text { otherwise }\end{cases}
$$

Then, for any family of real numbers $\left(\beta_{n}\right)_{1 \leq n \leq N}$ and for any real number $t$, the following identity holds

$$
\int_{\mathbb{R}}\left[\sum_{n=1}^{N} \beta_{n} \chi_{\tau}^{n}(t)\right] \mathrm{d} t=\tau \sum_{n=1}^{N} \beta_{n}
$$

As we have just seen, the estimation of space translates (see Theorem 4.20 ) does not use that $c_{\mathcal{T}}^{\Delta t}$ is solution to Problem 3.2). In fact this estimate is true for any $u_{\mathcal{T}} \in \mathbb{R}^{\mathcal{T}}$ and to obtain the convergence of approximate solutions we have to get the discrete $H^{1}$-bounds on 
the solution and on its trace (see assumption (4.20) of Proposition 4.22). Thus, if we consider another problem but whose discrete solutions also satisfy discrete $H^{1}$-bounds 4.20 , we can apply the previous results.

On the contrary to obtain estimation of time translates, we have to use that the couple $\left(c_{\mathcal{T}}^{\Delta t}, \mu_{\mathcal{T}}^{\Delta t}\right)$ is solution to the discrete Problem 3.2 and this estimate is specific to the studied system.

Now we give the estimate of time translates of the discrete order parameter which allows us to obtain the strong compactness that we lacked in Proposition 4.23 .

Thanks to the particular shape of the discrete extension operator which uses the trace of discrete solutions, the coupling between the domain and its boundary enable to obtain simultaneously the estimate both in $\Omega$ and on $\Gamma$.

THEOREM 4.26 (Estimation of time translates).

We assume that the assumptions of Theorem 4.4 are satisfied so that there exists a solution $\left(c_{\mathcal{T}}^{\Delta t}, \mu_{\mathcal{T}}^{\Delta t}\right)$ to discrete Problem (3.2) for all $N \in \mathbb{N}$ and for all admissible mesh $\mathcal{T}$.

Let $\tau>0$, then there exists $C_{11}>0$ independent of $\mathcal{T}, \Delta t$ and $\tau$ such that

$$
\left\|\zeta_{T}^{\mathcal{T}_{\alpha}}\left(c_{\mathcal{T}}^{\Delta t}\right)(.+\tau, .)-\zeta_{T}^{\mathcal{T}_{\alpha}}\left(c_{\mathcal{T}}^{\Delta t}\right)\right\|_{L^{2}\left(\mathbb{R} \times \mathbb{R}^{2}\right)}^{2} \leq C[1]^{\top} .
$$

We adapt the proof of Theorem A.2 in [14].

Proof.

Let $t \in \mathbb{R}$. Using Definition 4.24 of $\zeta_{T}^{\tau_{\alpha}}$ and definition 4.30) of $\chi_{\tau}^{n}$,

$$
\begin{aligned}
\zeta_{T}^{\tau_{\alpha}}\left(c_{\mathcal{T}}^{\Delta t}\right)(t+\tau, .)-\zeta_{T}^{\mathcal{T}_{\alpha}}\left(c_{\mathcal{T}}^{\Delta t}\right)(t, .)= & \chi_{\tau}^{0}(t) c_{\mathcal{T}_{\alpha}^{P}}^{1}-\chi_{\mathcal{\tau}}^{N}(t) c_{\mathcal{T}_{\alpha}^{P}}^{N} \\
& +\sum_{n=1}^{N-1} \chi_{\tau}^{n}(t)\left(c_{\mathcal{T}_{\alpha}^{P}}^{n+1}-c_{\mathcal{T}_{\alpha}^{P}}^{n}\right) .
\end{aligned}
$$

For any $s \in \mathbb{R}$, we define

$$
n(s)=\left\{\begin{array}{cl}
-2 & \text { if } s \leq 0 ; \\
\text { the index such that } t^{n(s)}<s \leq t^{n(s)+1} & \text { if } 0<s \leq t^{N} \\
N & \text { if } s>t^{N} .
\end{array}\right.
$$

Let $n_{0}(t)$ and $n_{1}(t)$ be given by $n_{0}(t)=n(t)$ and $n_{1}(t)=n(t+\tau)$. We adopt the convention $c_{\mathcal{T}}^{-1}=c_{\mathcal{T}}^{N+1}=0$. With this notation, equation 4.31) is equivalent to

$$
\zeta_{T}^{\tau_{\alpha}}\left(c_{\mathcal{T}}^{\Delta t}\right)(t+\tau, .)-\zeta_{T}^{\tau_{\alpha}}\left(c_{\mathcal{T}}^{\Delta t}\right)(t, .)=c_{\mathcal{T}_{\alpha}^{P}}^{n_{1}(t)+1}-c_{\mathcal{T}_{\alpha}^{P}}^{n_{0}(t)+1} .
$$

Gathering (4.31) and 4.33), we write

$$
\left.\int_{\mathbb{R}^{2}}\left(\zeta_{T}^{\mathcal{T}_{\alpha}}\left(c_{\mathcal{T}}^{\Delta t}\right)(t+\tau, x)-\zeta_{T}^{\mathcal{T}_{\alpha}}\left(c_{\mathcal{T}}^{\Delta t}\right)(t, x)\right)\right)^{2} \mathrm{~d} x=T_{1}(t)+T_{2}(t)+T_{3}(t)
$$

where:

$$
\begin{aligned}
& T_{1}(t)=\chi_{\tau}^{0}(t) \int_{\Omega^{P} \cup \Omega_{\alpha}^{P}}\left(c_{\tau_{\alpha}^{P}}^{n_{1}(t)+1}-c_{\mathcal{T}_{\alpha}^{P}}^{n_{0}(t)+1}\right) c_{\mathcal{T}_{\alpha}^{P}}^{1} \mathrm{~d} x:=T_{1}^{\Omega^{P}}(t)+T_{1}^{\Omega_{\alpha}^{P}}(t) \\
& T_{2}(t)=\sum_{n=1}^{N-1} \chi_{\tau}^{n}(t) \int_{\Omega^{P} \cup \Omega_{\alpha}^{P}}\left(c_{\mathcal{T}_{\alpha}^{P}}^{n_{1}(t)+1}-c_{\tau_{\alpha}^{P}}^{n_{0}(t)+1}\right)\left(c_{\mathcal{T}_{\alpha}^{P}}^{n+1}-c_{\mathcal{T}_{\alpha}^{P}}^{n}\right) \mathrm{d} x:=T_{2}^{\Omega^{P}}(t)+T_{2}^{\Omega_{\alpha}^{P}}(t) \\
& T_{3}(t)=-\chi_{\tau}^{N}(t) \int_{\Omega^{P} \cup \Omega_{\alpha}^{P}}\left(c_{\mathcal{T}_{\alpha}^{P}}^{n_{1}(t)+1}-c_{\mathcal{T}_{\alpha}^{P}}^{n_{0}(t)+1}\right) c_{\mathcal{T}_{\alpha}^{P}}^{N} \mathrm{~d} x:=T_{3}^{\Omega^{P}}(t)+T_{3}^{\Omega_{\alpha}^{P}}(t) .
\end{aligned}
$$


We begin with the terms $T_{\bullet}^{\Omega^{P}}(t)$ that concern contributions of $\Omega^{P}$.

Since $\chi_{\tau}^{0}(t)$ is equal to 1 on $\left[-\tau, 0\left[\right.\right.$ and 0 elsewhere, and since $c_{\mathfrak{M}}^{n_{0}(t)+1}=0$ for any negative $t$, we have

$$
\int_{\mathbb{R}} T_{1}^{\Omega^{P}}(t) \mathrm{d} t=\int_{-\tau}^{0} \int_{\Omega^{P}}\left(c_{\mathfrak{M}}^{n_{1}(t)+1}-c_{\mathfrak{M}}^{n_{0}(t)+1}\right) c_{\mathfrak{M}}^{1} \mathrm{~d} x \mathrm{~d} t=\int_{-\tau}^{0} \int_{\Omega^{P}} c_{\mathfrak{M}}^{n_{1}(t)+1} c_{\mathfrak{M}}^{1} \mathrm{~d} x \mathrm{~d} t,
$$

thus the definition of the discrete norm $\|\cdot\|_{0, \mathfrak{M}}$ implies

$$
\int_{\mathbb{R}} T_{1}^{\Omega^{P}}(t) \mathrm{d} t \leq \tau\left\|c_{\mathfrak{M}}^{\Delta t}\right\|_{L^{\infty}\left(0, T ;\|\cdot\|_{0, \mathfrak{M}}\right)}^{2}
$$

Similar arguments with $\chi_{\tau}^{N}$ and $c_{\mathfrak{M}}^{n_{1}(t)+1}$ give,

$$
\int_{\mathbb{R}} T_{3}^{\Omega^{P}}(t) \mathrm{d} t \leq \tau\left\|c_{\mathfrak{M}}^{\Delta t}\right\|_{L^{\infty}\left(0, T ;\|\cdot\|_{0, \mathfrak{M}}\right)}^{2} .
$$

Regarding the term $T_{2}^{\Omega^{P}}$, setting $v_{\mathcal{T}}=c_{\mathcal{T}}^{n_{1}(t)+1}-c_{\mathcal{T}}^{n_{0}(t)+1}$ as test function in equation 3.2a), then thanks to the Cauchy-Schwarz inequality, we have

$$
T_{2}^{\Omega^{P}}(t) \leq 2 \Gamma_{b}\left\|c_{\mathcal{T}}^{\Delta t}\right\|_{L^{\infty}\left(0, T ;\left.|\cdot|\right|_{1, \mathcal{T}}\right)} \sum_{n=1}^{N-1} \chi_{\mathcal{\tau}}^{n}(t) \Delta t\left|\mu_{\mathcal{T}}^{n+1}\right|_{1, \mathcal{\tau}} .
$$

Thanks to Lemma 4.25 and the Cauchy-Schwarz inequality we finally obtain,

$$
\int_{\mathbb{R}} T_{2}^{\Omega^{P}}(t) \mathrm{d} t \leq 2 \sqrt{T} \Gamma_{b} \tau\left\|c_{\mathcal{T}}^{\Delta t}\right\|_{L^{\infty}\left(0, T ;\left.|\cdot|\right|_{1}, \mathcal{T}\right)}\left\|\mu_{\mathcal{T}}^{\Delta t}\right\|_{L^{2}\left(0, T ;\left.|\cdot|\right|_{1, \mathcal{T}}\right)} .
$$

Now, we have to study the terms $T_{\bullet_{\alpha} \Omega^{P}}(t)$ concerning the contributions in $\Omega_{\alpha}^{P}$. The same arguments that for the term $T_{1}^{\Omega^{P}}(t)$ and Definition 4.17 of $c_{\mathfrak{M}_{\alpha}}$ give

$$
\int_{\mathbb{R}} T_{1}^{\Omega_{\alpha}}(t) \mathrm{d} t=\int_{-\tau}^{0} \sum_{\mathcal{R}_{\mathcal{L}}^{i} \in \mathfrak{M}_{\alpha}} m_{\mathcal{R}_{\mathcal{L}}^{i}} c_{\mathcal{L}}^{n_{1}(t)+1} c_{\mathcal{L}}^{1} \theta\left(d\left(x_{\mathcal{R}_{\mathcal{L}}^{i}}, \Gamma\right)\right)^{2} \mathrm{~d} t
$$

where $m_{\mathcal{R}_{\mathcal{L}}^{i}}$ is the Lebesgue measure of the quadrilateral whose vertices are those of the control volume $\mathcal{R}_{\mathcal{L}}^{i}$. Noticing that $\theta \leq 1$ and that there exists $C_{\Gamma, \alpha, \kappa}>0$ independent of $\operatorname{size}(\mathcal{T})$ such that $\sum_{i=1}^{n_{\alpha}} m_{\mathcal{R}_{\mathcal{L}}^{i}}=C_{\Gamma, \alpha, \kappa} m_{\mathbf{e}_{\mathcal{L}}}$, we obtain

$$
\int_{\mathbb{R}} T_{1}^{\Omega_{\alpha}^{P}}(t) \mathrm{d} t \leq C_{\Gamma, \kappa, \alpha} \tau\left\|c_{\partial \mathfrak{M}}^{\Delta t}\right\|_{L^{\infty}\left(0, T ;\|\cdot\|_{0, \partial \mathfrak{M})}^{2}\right.}^{2}
$$

A similar reasoning gets:

$$
\int_{\mathbb{R}} T_{3}^{\Omega_{\alpha}^{P}}(t) \mathrm{d} t \leq C_{\Gamma, \kappa, \alpha} \tau\left\|c_{\partial \mathfrak{M}}^{\Delta t}\right\|_{L^{\infty}\left(0, T ;\|\cdot\|_{0, \partial \mathfrak{M})}^{2}\right.} .
$$

As regards to the term $T_{2}^{\Omega_{\alpha}^{P}}$, thanks to Definition 4.17 of $c_{\mathfrak{m}_{\alpha}}$ we have

$$
T_{2}^{\Omega_{\alpha}^{P}}(t) \leq C_{\Gamma, \kappa, \alpha} \sum_{n=1}^{N-1} \chi_{\tau}^{n}(t) \sum_{\mathcal{L} \in \partial \mathfrak{M}} m_{\mathbf{e}_{\mathcal{L}}}\left(c_{\mathcal{L}}^{n_{1}(t)+1}-c_{\mathcal{L}}^{n_{0}(t)+1}\right)\left(c_{\mathcal{L}}^{n+1}-c_{\mathcal{L}}^{n}\right) .
$$


Setting $u_{\mathcal{T}}=c_{\mathcal{T}}^{n_{1}(t)+1}-c_{\mathcal{T}}^{n_{0}(t)+1}$ as test function in equations 3.2b) and 3.2c), we have

$$
T_{2}^{\Omega_{\alpha}^{P}}(t) \leq C_{\Gamma, \kappa, \alpha} \frac{\Delta t}{\mathcal{A}_{\partial t}}\left(\mathcal{A}_{\Delta_{\Gamma}} T_{2,1}^{\Omega_{\alpha}^{P}}(t)+\mathcal{A}_{\Delta} T_{2,2}^{\Omega_{\alpha}^{P}}(t)+\mathcal{A}_{f_{s}} T_{2,3}^{\Omega_{\alpha}^{P}}(t)+T_{2,4}^{\Omega_{\alpha}^{P}}(t)+\mathcal{A}_{f_{b}} T_{2,5}^{\Omega_{\alpha}^{P}}(t)\right)
$$

where

$$
\begin{aligned}
T_{2,1}^{\Omega_{\alpha}^{P}}(t) & =-\sum_{n=1}^{N-1} \chi_{\mathcal{\tau}}^{n}(t) \llbracket c_{\partial \mathfrak{M}}^{n+1}, c_{\partial \mathfrak{M}}^{n_{1}(t)+1}-c_{\partial \mathfrak{M}}^{n_{0}(t)+1} \rrbracket_{1, \partial \mathfrak{M}}, \\
T_{2,2}^{\Omega_{\alpha}^{P}}(t) & =-\sum_{n=1}^{N-1} \chi_{\mathcal{\tau}}^{n}(t) \llbracket c_{\mathcal{T}}^{n+1}, c_{\mathcal{T}}^{n_{1}(t)+1}-c_{\mathcal{T}}^{n_{0}(t)+1} \rrbracket_{1, \mathcal{T}}, \\
T_{2,3}^{\Omega_{\alpha}^{P}}(t) & =-\sum_{n=1}^{N-1} \chi_{\mathcal{\tau}}^{n}(t) \sum_{\mathcal{L} \in \partial \mathfrak{M}} m_{\mathbf{e}_{\mathcal{L}}} d^{f_{s}}\left(c_{\mathcal{L}}^{n}, c_{\mathcal{L}}^{n+1}\right)\left(c_{\mathcal{L}}^{n_{1}(t)+1}-c_{\mathcal{L}}^{n_{0}(t)+1}\right), \\
T_{2,4}^{\Omega_{\alpha}^{P}}(t) & =\sum_{n=1}^{N-1} \chi_{\tau}^{n}(t)\left(\mu_{\mathfrak{M}}^{n+1}, c_{\mathfrak{M}}^{n_{1}(t)+1}-c_{\mathfrak{M}}^{n_{0}(t)+1}\right)_{\mathfrak{M}}, \\
\text { and } \quad T_{2,5}^{\Omega_{\alpha}^{P}}(t) & =-\sum_{n=1}^{N-1} \chi_{\mathcal{\tau}}^{n}(t) \sum_{\mathcal{K} \in \mathfrak{M}} m_{\underline{\mathcal{K}}} d^{f_{b}}\left(c_{\mathcal{K}}^{n}, c_{\mathcal{K}}^{n+1}\right)\left(c_{\mathcal{K}}^{n_{1}(t)+1}-c_{\mathcal{K}}^{n_{0}(t)+1}\right) .
\end{aligned}
$$

Applying the Cauchy-Schwarz inequality, we obtain

$$
\begin{aligned}
& T_{2,1}^{\Omega_{\alpha}^{P}}(t) \leq 2\left\|c_{\partial \mathfrak{M}}^{\Delta t}\right\|_{L^{\infty}\left(0, T ;\left.|\cdot|\right|_{1, \partial \mathfrak{M}}\right)} \sum_{n=1}^{N-1} \chi_{\tau}^{n}(t)\left|c_{\partial \mathfrak{M}}^{n+1}\right|_{1, \partial \mathfrak{M}}, \\
& T_{2,2}^{\Omega_{\alpha}^{P}}(t) \leq 2\left\|c_{\mathcal{T}}^{\Delta t}\right\|_{L^{\infty}(0, T ;|\cdot| 1, \mathcal{T})} \sum_{n=1}^{N-1} \chi_{\mathcal{\tau}}^{n}(t)\left|c_{\mathcal{T}}^{n+1}\right|_{1, \mathcal{\tau}}, \\
& \text { and } \quad T_{2,4}^{\Omega_{\alpha}^{P}}(t) \leq 2\left\|c_{\mathfrak{M}}^{\Delta t}\right\|_{L^{\infty}\left(0, T ;\|\cdot\|_{0, \mathfrak{M}}\right)} \sum_{n=1}^{N-1} \chi_{\tau}^{n}(t)\left\|\mu_{\mathfrak{M}}^{n+1}\right\|_{0, \mathfrak{M}} .
\end{aligned}
$$

Using Sobolev inequality (4.3) on $\Gamma$, for any $n \in\{1, \ldots, N\}$ we have

$$
\left\|c_{\partial \mathfrak{M}}^{n}\right\|_{L^{\infty}(\Gamma)} \leq C_{3}\left\|c_{\partial \mathfrak{M}}^{n}\right\|_{1, \partial \mathfrak{M}},
$$

thus using bounds on the discrete solutions 4.77) and the Cauchy-Schwarz inequality, the term $T_{2,3}^{\Omega_{\alpha}^{P}}$ satisfies

$$
T_{2,3}^{\Omega_{\alpha}^{P}}(t) \leq 2 \sqrt{M_{\Gamma}} \max _{B\left(0, C_{\mathbb{Z}} M_{2}\right)}\left|f_{s}^{\prime}\right|\left\|c_{\partial \mathfrak{M}}^{\Delta t}\right\|_{L^{\infty}\left(0, T ;\|\cdot\|_{0, \partial \mathfrak{M})}\right.} \sum_{n=1}^{N-1} \chi_{\tau}^{n}(t) .
$$

Moreover, thanks to polynomial growth assumption (4.1), the Hölder inequality and PoincaréSobolev inequality 4.4,

$$
\begin{aligned}
T_{2,5}^{\Omega_{\alpha}^{P}}(t) \leq & 2 C_{b} \sqrt{M_{\Omega}}\left\|c_{\mathfrak{M}}^{\Delta t}\right\|_{L^{\infty}\left(0, T ;\|\cdot\|_{0, \mathfrak{M}}\right)} \sum_{n=1}^{N-1} \chi_{\mathcal{\tau}}^{n}(t) \\
& +2 C_{b} C_{2}^{p}\left\|c_{\mathcal{T}}^{\Delta t}\right\|_{L^{\infty}\left(0, T ;\|\cdot\|_{1, \mathcal{T}}\right)} \sum_{n=1}^{N-1} \chi_{\mathcal{\tau}}^{n}(t)\left(\left\|c_{\mathcal{T}}^{n}\right\|_{1, \mathcal{T}}^{p-1}+\left\|c_{\mathcal{T}}^{n+1}\right\|_{1, \mathcal{T}}^{p-1}\right) .
\end{aligned}
$$

Integrating the terms $T_{2, \bullet}^{\Omega_{\alpha}^{P}}(t)$ over the time and using Lemma 4.25 and the Hölder inequality, we obtain

$$
\begin{aligned}
& \Delta t \int_{\mathbb{R}} T_{2,1}^{\Omega_{\alpha}^{P}}(t) \leq 2 \sqrt{T} \tau\left\|c_{\partial \mathfrak{M}}^{\Delta t}\right\|_{L^{\infty}\left(0, T ;\left.|\cdot|\right|_{1, \partial \mathfrak{M}}\right)}\left\|c_{\partial \mathfrak{M}}^{\Delta t}\right\|_{L^{2}\left(0, T ;|\cdot|_{1, \partial \mathfrak{M})}\right.}, \\
& \Delta t \int_{\mathbb{R}} T_{2,2}^{\Omega_{\alpha}^{P}}(t) \leq 2 \sqrt{T} \tau\left\|c_{\mathcal{T}}^{\Delta t}\right\|_{L^{\infty}\left(0, T ;|\cdot|_{1, \mathcal{T}}\right)}\left\|c_{\mathcal{T}}^{\Delta t}\right\|_{L^{2}\left(0, T ;\left.|\cdot|\right|_{1, \mathcal{T}}\right)}, \\
& \Delta t \int_{\mathbb{R}} T_{2,3}^{\Omega_{\alpha}^{P}}(t) \leq 2 \sqrt{M_{\Gamma}} T \tau \max _{B\left(0, C_{\mathfrak{Z}} M_{2}\right)}\left|f_{s}^{\prime}\right|\left\|c_{\partial \mathfrak{M}}^{\Delta t}\right\|_{L^{\infty}\left(0, T ;\|\cdot\|_{0, \partial \mathfrak{M})}\right.},
\end{aligned}
$$




$$
\begin{aligned}
& \Delta t \int_{\mathbb{R}} T_{2,4}^{\Omega_{\alpha}^{P}}(t) \leq 2 \sqrt{T} \tau\left\|c_{\mathfrak{M}}^{\Delta t}\right\|_{L^{\infty}\left(0, T ;\|\cdot\|_{0, \mathfrak{M})}\right.}\left\|\mu_{\mathfrak{M}}^{\Delta t}\right\|_{L^{2}\left(0, T ;\|\cdot\|_{0, \mathfrak{M}}\right)}, \\
& \text { and } \begin{aligned}
\Delta t \int_{\mathbb{R}} T_{2,5}^{\Omega_{\alpha}^{P}}(t) \leq & 2 C_{b} \sqrt{M_{\Omega}} T \tau\left\|c_{\mathfrak{M}}^{\Delta t}\right\|_{L^{\infty}\left(0, T ;\|\cdot\|_{0, \mathfrak{M})}\right)} \\
& +4 C_{b} C_{2}^{p} T^{\frac{1}{p}}\left\|c_{\mathcal{T}}^{\Delta t}\right\|_{L^{\infty}\left(0, T ;\|\cdot\|_{1, \mathcal{T})}\right.}\left\|c_{\mathcal{T}}^{\Delta t}\right\|_{L^{p}\left(0, T ;\|\cdot\|_{1, \mathcal{T}}\right)}^{p-1} .
\end{aligned}
\end{aligned}
$$

Gathering the equations (4.34)-(4.44) and using bounds on the solutions 4.77 complete the proof.

4.3.5. Proof of the convergence result. We are now in position to complete the proof of Theorem 4.6 We split the proof in three steps. First, we use the previous results to prove the existence of the limits. Then, we study the passage to the limit for the time evolution equation (1.1a) in $\Omega$ which is easier because there are no non-linear terms. Finally, we focus on the passage to the limit for the equation on the chemical potential (1.1b) using the equation on $\Gamma$ given by the dynamic boundary condition (1.4).

\section{Step 1: Existence of the limits}

Using the estimate of space and time translates (see Theorems 4.20 and 4.26) and the bounds on the discrete solutions (see Proposition 4.11), there exists $M>0$ independent of $\operatorname{size}(\mathcal{T}), \Delta t, \eta$ and $\tau$ such that,

$$
\begin{aligned}
\left\|\zeta_{T}^{\tau_{\alpha}}\left(c_{\mathcal{T}}^{\Delta t}\right)(., .+\eta)-\zeta_{T}^{\tau_{\alpha}}\left(c_{\mathcal{T}}^{\Delta t}\right)\right\|_{L^{2}\left(\mathbb{R} \times \mathbb{R}^{2}\right)}^{2} & \leq M|\eta|(|\eta|+\operatorname{size}(\mathcal{T})) \\
\text { and } \quad\left\|\zeta_{T}^{\mathcal{T}_{\alpha}}\left(c_{\mathcal{T}}^{\Delta t}\right)(.+\tau, .)-\zeta_{T}^{\tau_{\alpha}}\left(c_{\mathcal{\mathcal { T }}}^{\Delta t}\right)\right\|_{L^{2}\left(\mathbb{R} \times \mathbb{R}^{2}\right)}^{2} & \leq M \tau .
\end{aligned}
$$

Moreover, Definition 4.24 of the extension operator $\zeta_{T}^{\tau_{\alpha}}$ and Proposition 4.21 associated with the bounds on the discrete solutions imply

$$
\left\|\zeta_{T}^{\mathcal{T}_{\alpha}}\left(c_{\mathcal{T}}^{\Delta t}\right)\right\|_{L^{2}\left(\mathbb{R} \times \mathbb{R}^{2}\right)}^{2} \leq M
$$

Thus, we can apply the Kolmogorov theorem and there exists a function $\widetilde{c} \in L^{2}\left((0, T) \times \mathbb{R}^{2}\right)$ such that, up to a subsequence, the following convergence holds

$$
\zeta_{T}^{\mathcal{T}_{\alpha}}\left(c_{\mathcal{T}}^{\Delta t}\right) \underset{m \rightarrow+\infty}{\longrightarrow} \widetilde{c} \text { in } L^{2}\left((0, T) \times \mathbb{R}^{2}\right) .
$$

Thanks to Proposition 4.23 the subsequence $\left(\left(c_{\mathcal{T}}^{\Delta t}\right)^{(m)}\right)_{m \in \mathbb{N}}$ strongly converges in $L^{2}\left(0, T ; L^{2}(\Omega)\right)$ towards the function $c=\widetilde{c}_{\mid \Omega} \in L^{\infty}\left(0, T, H^{1}(\Omega)\right)$ and the subsequence $\left(\left(c_{\partial \mathfrak{M}}^{\Delta t}\right)^{(m)}\right)_{m \in \mathbb{N}}$ strongly converges in $L^{2}\left(0, T ; L^{2}(\Gamma)\right)$ towards $c_{1 \Gamma}=\operatorname{Tr} c \in L^{\infty}\left(0, T ; H^{1}(\Gamma)\right)$. Furthermore, the subsequence $\left(\left(\nabla^{\mathcal{T}} c_{\mathcal{T}}^{\Delta t}\right)^{(m)}\right)_{m \in \mathbb{N}}$ weakly converges towards $\nabla c$ in $L^{2}\left(0, T ; L^{2}(\Omega)\right)$ and $\left(\left(\nabla_{\Gamma}^{\partial \mathfrak{M}} c_{\partial \mathfrak{M}}^{\Delta t}\right)^{(m)}\right)_{m \in \mathbb{N}}$ weakly converges towards $\nabla_{\Gamma} c_{\Gamma \Gamma}$ in $L^{2}\left(0, T ; L^{2}(\Gamma)\right)$. Applying Proposition 4.13 together with Proposition 4.11 , the sequence $\left(\left(\mu_{\mathcal{T}}^{\Delta t}\right)^{(m)}\right)_{m \in \mathbb{N}}$ weakly converges, up to a subsequence, in $L^{2}\left(0, T, L^{q}(\Omega)\right)(q \geq 1)$ towards a certain $\mu \in$ $L^{2}\left(0, T, H^{1}(\Omega)\right)$.

In the sequel, for enhanced readability, the superscript ${ }^{(m)}$ will be omitted.

Step 2: Convergence for the equation (4.5)

Let $\phi \in \mathcal{C}^{2,1}\left(\mathbb{R} \times \mathbb{R}^{2}\right)$ such that $\phi(T,)=$.0 and $\nabla \phi \cdot \overrightarrow{\mathbf{n}}=0$ on $(0, T) \times \Gamma$. Since $\Omega$ is an open subset of $\mathbb{R}^{2}$ with a $\mathcal{C}^{3,1}$-continuous boundary, the set of such function $\phi$ is dense for the norm $L^{2}\left(0, T, H^{1}(\Omega)\right)$ in the set of function $\widetilde{\phi} \in \mathcal{C}^{2}\left(\mathbb{R} \times \mathbb{R}^{2}\right)$ satisfying $\widetilde{\phi}(T,)=$.0 (see [9]). 
Multiplying equation 3.2a by $\phi_{\mathcal{K}}^{n}:=\phi\left(t^{n}, x_{\mathcal{K}}\right)$ and summing up over the interior control volumes and the times steps, we get

$$
\sum_{n=0}^{N-1} \sum_{\mathcal{K} \in \mathfrak{M}} \phi_{\mathcal{K}}^{n+1}\left[m_{\underline{\mathcal{K}}}\left(c_{\mathcal{K}}^{n+1}-c_{\mathcal{K}}^{n}\right)+\Delta t \Gamma_{b} \sum_{\sigma \in \mathcal{E}_{\mathcal{K}}^{n t}} m_{\sigma}\left(\frac{\mu_{\mathcal{K}}^{n+1}-\mu_{\mathcal{L}}^{n+1}}{d_{\mathcal{K}, \mathcal{L}}}\right)\right]=0 .
$$

The first term reads

$$
T_{1}=-\sum_{\mathcal{K} \in \mathfrak{M}} m_{\underline{\mathcal{K}}} c_{\mathcal{K}}^{0} \phi_{\mathcal{K}}^{1}+\sum_{n=1}^{N-1} \sum_{\mathcal{K} \in \mathfrak{M}} m_{\underline{\mathcal{K}}} c_{\mathcal{K}}^{n}\left(\phi_{\mathcal{K}}^{n}-\phi_{\mathcal{K}}^{n+1}\right)+\sum_{\mathcal{K} \in \mathfrak{M}} m_{\underline{\mathcal{K}}} \underbrace{\phi_{\mathcal{K}}^{N}}_{=\phi\left(T, x_{\mathcal{K}}\right)=0} c_{\mathcal{K}}^{N},
$$

and using a similar reasoning to this given for example in [14, Theorem 4.2], we can prove

$$
T_{1} \underset{\Delta t, \operatorname{size}(\mathcal{T}) \rightarrow 0}{\longrightarrow}-\int_{\Omega} c^{0}(x) \phi(0, x) \mathrm{d} x-\int_{0}^{T} \int_{\Omega} c(t, x) \partial_{t} \phi(t, x) \mathrm{d} x \mathrm{~d} t .
$$

Reordering the summations, the second term of 4.45 can be written,

$$
T_{2}=\Gamma_{b} \sum_{n=0}^{N-1} \Delta t \sum_{\mathcal{K} \in \mathfrak{M}} \mu_{\mathcal{K}}^{n+1} \sum_{\sigma \in \mathcal{E}_{\mathcal{K}}^{i n t}} m_{\sigma}\left(\frac{\phi_{\mathcal{K}}^{n+1}-\phi_{\mathcal{L}}^{n+1}}{d_{\mathcal{K}, \mathcal{L}}}\right) .
$$

Since $\nabla \phi \cdot \overrightarrow{\mathbf{n}}=0$ on $(0, T) \times \Omega$, for any $t \in \mathbb{R}$ the Stokes formula gives

$$
\int_{\mathcal{K}} \Delta \phi(t, x) \mathrm{d} x=\int_{\partial \mathcal{K}} \nabla \phi(t, x) \cdot \overrightarrow{\mathbf{n}} \mathrm{d} \sigma(x)=\sum_{\sigma \in \mathcal{E}_{\mathcal{K}}^{\text {int }}} \int_{\sigma} \nabla \phi(t, x) \cdot \overrightarrow{\mathbf{n}}_{\mathcal{K} \sigma} \mathrm{d} \sigma(x) .
$$

Then,

$$
\begin{aligned}
T_{2}= & -\Gamma_{b} \int_{0}^{T} \int_{\Omega} \Delta \phi(t, x) \mu_{\mathfrak{M}}^{\Delta t}(t, x) \mathrm{d} x \\
& +\Gamma_{b} \sum_{n=0}^{N-1} \Delta t \sum_{\sigma=\mathcal{K} \mid \mathcal{\mathcal { L }} \in \mathcal{E}_{i n t}}\left(\mu_{\mathcal{K}}^{n+1}-\mu_{\mathcal{L}}^{n+1}\right) R_{\sigma, n+1}^{\phi}
\end{aligned}
$$

where

$$
R_{\sigma, n+1}^{\phi}=\frac{1}{m_{\sigma} \Delta t} \int_{t^{n}}^{t^{n+1}} \int_{\sigma} \nabla \phi(t, x) \cdot \overrightarrow{\mathbf{n}}_{\mathcal{K} \sigma} \mathrm{d} \sigma(x) \mathrm{d} t-\frac{\phi_{\mathcal{L}}^{n+1}-\phi_{\mathcal{K}}^{n+1}}{d_{\mathcal{K}, \mathcal{L}}} .
$$

The regularity of the function $\phi$ and the bounds on the discrete solutions imply that the second term of (4.47) tends to 0 . Moreover, $\mu_{\mathcal{T}}^{\Delta t}$ weakly converges (up to a subsequence) in $L^{2}\left(0, T ; L^{2}(\Omega)\right)$ towards $\mu \in L^{2}\left(0, T ; H^{1}(\Omega)\right)$ and $\nabla \phi \cdot \overrightarrow{\mathbf{n}}=0$ on $(0, T) \times \Omega$, thus

$$
T_{2} \underset{\Delta t, \operatorname{size}(\mathcal{T}) \rightarrow 0}{\longrightarrow} \Gamma_{b} \int_{0}^{T} \int_{\Omega} \nabla \phi(t, x) \cdot \nabla \mu(t, x) \mathrm{d} x \mathrm{~d} t .
$$

Finally, since $T_{1}+T_{2}=0$ the convergences (4.46) and (4.49) allow to obtain the following equality for any $\phi \in \mathcal{C}^{2,1}\left(\mathbb{R} \times \mathbb{R}^{2}\right)$ such that $\phi(T,)=$.0 and $\nabla \phi \cdot \overrightarrow{\mathbf{n}}=0$ on $(0, T) \times \Gamma$.

$$
\int_{0}^{T} \int_{\Omega}\left(-c(t, x) \partial_{t} \phi(t, x)+\Gamma_{b} \nabla \phi(t, x) \cdot \nabla \mu(t, x)\right) \mathrm{d} x \mathrm{~d} t=\int_{\Omega} c^{0}(x) \phi(0, x) \mathrm{d} x
$$


By density, we obtain the equality 4.50 for any function $\phi \in \mathcal{C}^{2}\left(\mathbb{R} \times \mathbb{R}^{2}\right)$ satisfying $\phi(T,)=0$.

Step 3: Convergence for the equation (4.6)

Let $\phi \in \mathcal{C}^{2}\left(\mathbb{R} \times \mathbb{R}^{2}\right)$ such that $\phi(T,)=$.0 . First, multiplying equation $3.2 \mathrm{~b}$ by $\Delta t \phi_{\mathcal{K}}^{n+1}$ and summing up over all interior control volume $\mathcal{K} \in \mathfrak{M}$ and over all time interval $\left[t^{n}, t^{n+1}\right]$. Secondly, multiplying equation $(3.2 \mathrm{c})$ by $\phi_{\mathcal{L}}^{n+1}:=\phi\left(t^{n+1}, x_{\mathcal{L}}\right)$ and summing up over all boundary control volumes $\mathcal{L} \in \partial \mathfrak{M}$ and over all time interval. Then, summing the two identities we get

$$
\mathcal{A}_{\Delta} T_{1}+T_{2}+\mathcal{A}_{f_{b}} T_{3}+\mathcal{A}_{\partial t} T_{4}+\mathcal{A}_{\Delta_{\Gamma}} T_{5}+\mathcal{A}_{f_{s}} T_{6}=0
$$

where

$$
\begin{aligned}
T_{1}= & \sum_{n=0}^{N-1} \Delta t\left[\sum_{\mathcal{K} \in \mathfrak{M}} \phi_{\mathcal{K}}^{n+1}\left(\sum_{\sigma \in \mathcal{E}_{\mathcal{K}}^{i n t}} m_{\sigma}\left(\frac{c_{\mathcal{K}}^{n+1}-c_{\mathcal{L}}^{n+1}}{d_{\mathcal{K}, \mathcal{L}}}\right)+\sum_{\sigma \in \mathcal{E}_{\mathcal{K}}^{e x t}} m_{\mathbf{e}_{\mathcal{L}}}\left(\frac{c_{\mathcal{K}}^{n+1}-c_{\mathcal{L}}^{n+1}}{d_{\mathcal{K}, \mathcal{L}}}\right)\right)\right. \\
& \left.+\sum_{\sigma=\mathcal{L} \in \mathcal{E}_{\text {ext }}} \phi_{\mathcal{L}}^{n+1} m_{\mathbf{e}_{\mathcal{L}}} \frac{c_{\mathcal{L}}^{n+1}-c_{\mathcal{K}}^{n+1}}{d_{\mathcal{K}, \mathcal{L}}}\right], \\
T_{2}= & -\sum_{n=0}^{N-1} \Delta t \sum_{\mathcal{K} \in \mathfrak{M}} m_{\underline{\mathcal{K}}} \phi_{\mathcal{K}}^{n+1} \mu_{\mathcal{K}}^{n+1}, \quad T_{5}=\sum_{n=0}^{N-1} \Delta t \sum_{\sigma=\mathcal{L} \in \mathcal{E}_{\text {ext }}} \phi_{\mathcal{L}}^{n+1} \sum_{\mathbf{v} \in \mathcal{V}_{\mathcal{L}}}\left(\frac{c_{\mathcal{L}}^{n+1}-c_{\mathcal{L}^{\prime}}^{n+1}}{d_{\mathcal{L}, \mathcal{L}^{\prime}}}\right), \\
T_{3}= & \sum_{n=0}^{N-1} \Delta t \sum_{\mathcal{K} \in \mathfrak{M}} m_{\underline{\mathcal{K}}} \phi_{\mathcal{K}}^{n+1} d^{f_{b}}\left(c_{\mathcal{K}}^{n}, c_{\mathcal{K}}^{n+1}\right), \quad T_{6}=\sum_{n=0}^{N-1} \Delta t \sum_{\sigma=\mathcal{L} \in \mathcal{E}_{\text {ext }}} m_{\mathbf{e}_{\mathcal{L}}} \phi_{\mathcal{L}}^{n+1} d^{f_{s}}\left(c_{\mathcal{L}}^{n}, c_{\mathcal{L}}^{n+1}\right), \\
T_{4}= & \sum_{n=0}^{N-1} \sum_{\sigma=\mathcal{L} \in \mathcal{E}_{\text {ext }}} m_{\mathbf{e}_{\mathcal{L}} \phi_{\mathcal{L}}^{n+1}\left(c_{\mathcal{L}}^{n+1}-c_{\mathcal{L}}^{n}\right) .}
\end{aligned}
$$

We begin by the term $T_{1}$. By reordering the summations and noting that for any $\mathcal{K} \in \mathfrak{M}$ and for any $t \in \mathbb{R}$,

$$
\int_{\mathcal{K}} \Delta \phi(t, x) \mathrm{d} x-\sum_{\sigma \in \mathcal{E}_{\mathcal{K}}} \int_{\sigma} \nabla \phi(t, x) \cdot \overrightarrow{\mathbf{n}}_{\mathcal{K} \boldsymbol{\sigma}}(x) \mathrm{d} \sigma(x)=0
$$

we get

$$
\begin{aligned}
T_{1}= & -\int_{0}^{T} \int_{\Omega} c_{\mathfrak{M}}^{\Delta t} \Delta \phi(t, x) \mathrm{d} x \mathrm{~d} t+\int_{0}^{T} \int_{\Gamma} c_{\partial \mathfrak{M}}^{\Delta t} \nabla \phi(t, x) \cdot \overrightarrow{\mathbf{n}}(x) \mathrm{d} \sigma(x) \mathrm{d} t \\
& +\sum_{n=0}^{N-1} \Delta t \sum_{\sigma=\mathcal{K} \mid \mathcal{L} \in \mathcal{E}_{i n t}} m_{\sigma}\left(c_{\mathcal{K}}^{n+1}-c_{\mathcal{L}}^{n+1}\right) R_{\sigma, n+1}^{\phi} \\
& +\sum_{n=0}^{N-1} \Delta t \sum_{\sigma=\mathcal{L} \in \mathcal{E}_{\text {ext }}} m_{\mathbf{e}_{\mathcal{L}}}\left(c_{\mathcal{K}}^{n+1}-c_{\mathcal{L}}^{n+1}\right) R_{\sigma, n+1}^{\phi}
\end{aligned}
$$

where $R_{\sigma, n+1}^{\phi}$ is defined by 4.48 .

Using the regularity of the function $\phi$ and the bounds on the discrete solutions the two last terms of the right hand side of (4.52) tend to 0 . Furthermore, $c_{\mathfrak{m}}^{\Delta t}$ converges in $L^{2}\left(0, T ; L^{2}(\Omega)\right)$ towards $c \in L^{2}\left(0, T ; H^{1}(\Omega)\right)$ and $c_{\partial \mathfrak{M}}^{\Delta t}$ converges towards $c_{\perp \Gamma}$ in $L^{2}\left(0, T ; L^{2}(\Gamma)\right)$, so

$$
T_{1} \underset{\Delta t, \operatorname{size}(\mathcal{T}) \rightarrow 0}{\longrightarrow} \int_{0}^{T} \int_{\Omega} \nabla c(t, x) \cdot \nabla \phi(t, x) \mathrm{d} x \mathrm{~d} t .
$$

Let us now turn to the term $T_{2}$,

$$
T_{2}=-\int_{0}^{T} \int_{\Omega} \phi(t, x) \mu_{\mathfrak{M}}^{\Delta t} \mathrm{~d} x \mathrm{~d} t+\sum_{n=0}^{N-1} \Delta t \sum_{\mathcal{K} \in \mathfrak{M}} m_{\underline{\mathcal{K}}} \mu_{\mathcal{K}}^{n+1} R_{\mathcal{K}, n}^{\phi}
$$


where

$$
R_{\mathcal{K}, n}^{\phi}=\frac{1}{\Delta t m_{\underline{\mathcal{K}}}} \int_{t^{n}}^{t^{n+1}} \int_{\mathcal{K}} \phi(t, x) \mathrm{d} x \mathrm{~d} t-\phi\left(t^{n+1}, x_{\mathcal{K}}\right)
$$

Since $\mu_{\mathfrak{m}}^{\Delta t}$ weakly converges in $L^{2}\left(0, T ; L^{2}(\Omega)\right)$ towards $\mu$ and noting that the second term of the right hand side of $T_{2}$ tends to 0 , we get

$$
T_{2} \underset{\Delta t, \operatorname{size}(\mathcal{T}) \rightarrow 0}{\longrightarrow}-\int_{0}^{T} \int_{\Omega} \phi(t, x) \mu(t, x) \mathrm{d} x \mathrm{~d} t .
$$

Considering now the term $T_{3}$. Recalling that for any $x \in \mathbb{R}, d^{f_{b}}(x, x)=f_{b}^{\prime}(x)$ thus

$$
\begin{aligned}
T_{3}= & \sum_{n=0}^{N-1} \Delta t \sum_{\mathcal{K} \in \mathfrak{M}} m_{\underline{\mathcal{K}}} \phi_{\mathcal{K}}^{n+1}\left(d^{f_{b}}\left(c_{\mathcal{K}}^{n}, c_{\mathcal{K}}^{n+1}\right)-d^{f_{b}}\left(c_{\mathcal{K}}^{n+1}, c_{\mathcal{K}}^{n+1}\right)\right) \\
& +\sum_{n=0}^{N-1} \Delta t \sum_{\mathcal{K} \in \mathfrak{M}} m_{\underline{\mathcal{K}}} \phi_{\mathcal{K}}^{n+1} f_{b}^{\prime}\left(c_{\mathcal{K}}^{n+1}\right) .
\end{aligned}
$$

Then, the first term in the right hand side of $T_{3}$ satisfies

$$
\left|T_{3,1}\right| \leq \widetilde{C_{b}} \sum_{n=0}^{N-1} \Delta t \sum_{\mathcal{K} \in \mathfrak{M}} m_{\underline{\mathcal{K}}}\left|\phi_{\mathcal{K}}^{n+1}\right|\left|c_{\mathcal{K}}^{n+1}-c_{\mathcal{K}}^{n}\right|\left(1+\left|c_{\mathcal{K}}^{n}\right|^{p-2}+\left|c_{\mathcal{K}}^{n+1}\right|^{p-2}\right)
$$

because the assumption 4.1) gives

$$
\begin{aligned}
\left|d^{f_{b}}(a, b)-d^{f_{b}}(a, a)\right| & \leq\left(\sup _{s \in[0,1]}\left|D\left(d^{f_{b}}(a, \cdot)\right)(s a+(1-s) b)\right|\right)|b-a| \\
& \leq C_{b}\left(1+|a|^{p-2}+\sup _{s \in[0,1]}|s a+(1-s) b|^{p-2}\right)|b-a| \\
& \leq \widetilde{C_{b}}\left(1+|a|^{p-2}+|b|^{p-2}\right)|b-a| .
\end{aligned}
$$

Hence, thanks to the Hölder inequality, we get

$$
\left|T_{3,1}\right| \leq \widetilde{C}_{b} \sum_{n=0}^{N-1} \Delta t\left\|\phi_{\mathfrak{M}}^{n+1}\right\|_{0, p, \mathfrak{M}}\left\|c_{\mathfrak{M}}^{n+1}-c_{\mathfrak{M}}^{n}\right\|_{0, p, \mathfrak{M}}\left\|1+\left|c_{\mathfrak{M}}^{n}\right|^{p-2}+\left|c_{\mathfrak{M}}^{n+1}\right|^{p-2}\right\|_{0, \frac{p}{p-2}, \mathfrak{M}},
$$

thus thanks to Poincaré-Sobolev inequality (4.4) and bounds on the solutions 4.7) the term $T_{3,1}$ tends to 0 when the time step tends to 0 .

With regard to the second term in the right hand side of $T_{3}$, we have

$$
T_{3,2}=\int_{0}^{T} \int_{\Omega} \phi(t, x) f_{b}^{\prime}\left(c_{\mathfrak{M}}^{\Delta t}\right) \mathrm{d} x \mathrm{~d} t-\sum_{n=0}^{N-1} \Delta t \sum_{\mathcal{K} \in \mathfrak{M}} m_{\underline{\mathcal{K}}} f_{b}^{\prime}\left(c_{\mathcal{K}}^{n+1}\right) R_{\mathcal{K}, n}^{\phi}
$$

where $R_{\mathcal{K}, n}^{\phi}$ is defined by the relation (4.54).

We begin with the first term in the right hand side of $T_{3,2}$.

On one hand, for any $r \geq 1$, thanks to Poincaré-Sobolev inequality (4.4) and bounds on the solutions 4.7), we have

$$
\left\|c_{\mathfrak{M}}^{\Delta t}\right\|_{L^{2}\left(0, T ;\|\cdot\|_{0, r, \mathfrak{M})}\right.}^{2} \leq C_{2}^{2}\left\|c_{\mathcal{T}}^{\Delta t}\right\|_{L^{2}\left(0, T ;\|\cdot\|_{1, \mathcal{T}}\right.}^{2} \leq C_{2}^{2} T M_{1}^{2} .
$$

Then, $c_{\mathfrak{M}}^{\Delta t}$ weakly converges (up to a subsequence) towards a certain $\widetilde{c}$ in $L^{2}\left(0, T ; L^{r}(\Omega)\right.$ ). But, we know that $c_{\mathfrak{m}}^{\Delta t}$ strongly converges in $L^{2}\left(0, T ; L^{2}(\Omega)\right)$ towards $c \in L^{2}\left(0, T ; H^{1}(\Omega)\right)$. 
Thus, $\widetilde{c}=c$ and $c_{\mathfrak{M}}^{\Delta t}$ weakly converges (up to a subsequence) in $L^{2}\left(0, T ; L^{r}(\Omega)\right.$ ) towards $c$. On the other hand, for any $u_{\mathfrak{M}} \in \mathbb{R}^{\mathfrak{M}}$ and for any $\left.\left.s \in\right] 0,1\right]$ the Hölder inequality gives

$$
\|u\|_{L^{r}(\Omega)} \leq\|u\|_{L^{p}(\Omega)}^{s}\|u\|_{L^{q}(\Omega)}^{1-s} \text { with } \frac{1}{r}=\frac{s}{p}+\frac{1-s}{q},
$$

and with $p=2$, for any $t \in \mathbb{R}$ we have

$$
\left\|c_{\mathfrak{M}}^{\Delta t}(t, \cdot)-c(t, \cdot)\right\|_{L^{r}(\Omega)} \leq\left\|c_{\mathfrak{M}}^{\Delta t}(t, \cdot)-c(t, \cdot)\right\|_{L^{2}(\Omega)}^{s}\left\|c_{\mathfrak{M}}^{\Delta t}(t, \cdot)-c(t, \cdot)\right\|_{L^{q}(\Omega)}^{1-s}
$$

and thus,

$$
\left\|c_{\mathfrak{M}}^{\Delta t}-c\right\|_{L^{2}\left(0, T ; L^{r}(\Omega)\right)}^{2} \leq\left\|c_{\mathfrak{M}}^{\Delta t}-c\right\|_{L^{2}\left(0, T ; L^{2}(\Omega)\right)}^{2 s}\left\|c_{\mathfrak{M}}^{\Delta t}-c\right\|_{L^{2}\left(0, T ; L^{q}(\Omega)\right)}^{2(1-s)} .
$$

Since, $c_{\mathfrak{M}}^{\Delta t}$ strongly converges in $L^{2}\left(0, T ; L^{2}(\Omega)\right)$ towards $c$, we have

$$
\left\|c_{\mathfrak{M}}^{\Delta t}-c\right\|_{L^{2}\left(0, T ; L^{r}(\Omega)\right)}^{2} \underset{\Delta t, \operatorname{size}(\mathcal{T}) \rightarrow 0}{\longrightarrow} 0
$$

and thus together with the weak convergence of $c_{\mathfrak{M}}^{\Delta t}$ in $L^{2}\left(0, T ; L^{r}(\Omega)\right)$, we conclude that $c_{\mathfrak{M}}^{\Delta t}$ strongly converges in $L^{2}\left(0, T ; L^{r}(\Omega)\right)$ towards $c$. Thus, up to a subsequence we have

$$
\left\{\begin{aligned}
c_{\mathfrak{M}}^{\Delta t}(t, x) & \rightarrow c(t, x) \text { a.e in }(0, T) \times \Omega, \\
\left|c_{\mathfrak{M}}^{\Delta t}(t, x)\right| \leq S(t, x) & \text { a.e. in }(0, T) \times \Omega \text { with } S \in L^{2}\left(0, T ; L^{r}(\Omega)\right) .
\end{aligned}\right.
$$

Then, thanks to polynomial growth assumption 1.6 ,

$$
\left|f_{b}^{\prime}\left(c_{\mathfrak{M}}^{\Delta t}(t, x)\right) \phi(t, x)\right| \leq C_{b}\left(1+|S(t, x)|^{p-1}\right)|\phi(t, x)| \in L^{1}(0, T ; \Omega) .
$$

Thus, using the dominated convergence theorem and relation 4.56, we obtain

$$
\int_{0}^{T} \int_{\Omega} f_{b}^{\prime}\left(c_{\mathfrak{M}}^{\Delta t}(t, x)\right) \phi(t, x) \mathrm{d} x \mathrm{~d} t \underset{\Delta t, \operatorname{size}(\mathcal{T}) \rightarrow 0}{\longrightarrow} \int_{0}^{T} \int_{\Omega} f_{b}^{\prime}(c(t, x)) \phi(t, x) \mathrm{d} x \mathrm{~d} t .
$$

Owing to polynomial growth assumption (1.6), Sobolev-Poincaré inequality (4.4) and bounds on the solutions (4.7), the second term in the right hand side of $T_{3,2}$ tends to 0 , thus

$$
T_{3} \underset{\Delta t, \operatorname{size}(\mathcal{T}) \rightarrow 0}{\longrightarrow} \int_{0}^{T} \int_{\Omega} f_{b}^{\prime}(c(t, x)) \phi(t, x) \mathrm{d} x \mathrm{~d} t .
$$

Let us now study the convergence of the term $T_{4}$.

$$
T_{4}=-\sum_{\mathcal{L} \in \partial \mathfrak{M}} m_{\mathbf{e}_{\mathcal{L}}} c_{\mathcal{L}}^{0} \phi_{\mathcal{L}}^{1}+\sum_{n=1}^{N-1} \sum_{\mathcal{L} \in \partial \mathfrak{M}} m_{\mathbf{e}_{\mathcal{L}}} c_{\mathcal{L}}^{n}\left(\phi_{\mathcal{L}}^{n}-\phi_{\mathcal{L}}^{n+1}\right)+\sum_{\mathcal{L} \in \partial \mathfrak{M}} m_{\mathbf{e}_{\mathcal{L}}} \underbrace{\phi_{\mathcal{L}}^{N}}_{\phi\left(T, x_{\mathcal{L}}\right)=0} c_{\mathcal{L}}^{N} .
$$

As for the term $T_{1}$ in the step 2, we use a similar reasoning to this given in [14, Theorem 4.2] to obtain,

$$
T_{4} \underset{\Delta t, \operatorname{size}(\mathcal{T}) \rightarrow 0}{\longrightarrow}-\int_{0}^{T} \int_{\Gamma} c_{i \Gamma}(t, x) \partial_{t} \phi(t, x) \mathrm{d} \sigma(x) \mathrm{d} t-\int_{\Gamma} \operatorname{Tr} c^{0}(x) \phi(0, x) \mathrm{d} \sigma(x) .
$$

Now, we focus on the term $T_{5}$. Reordering the terms and noting that for any $\mathcal{L} \in \partial \mathfrak{M}$,

$$
\int_{\mathcal{L}} \Delta_{\Gamma} \phi(t, x) \mathrm{d} \sigma(x)=\sum_{\mathbf{v} \in \mathcal{V}_{\mathcal{L}}} \nabla_{\Gamma} \phi(t, \mathbf{v}) \cdot \overrightarrow{\boldsymbol{\tau}}_{\mathbf{v}, \mathcal{L}}, \forall t \in \mathbb{R}
$$


where $\vec{\tau}_{\mathrm{v}, \mathcal{L}}=n_{\mathbf{v} \mathcal{L}} \vec{\tau}$ is the unit tangent vector going from $\mathcal{L}$ to $\mathcal{L}^{\prime}$; we have

$$
T_{5}=-\int_{0}^{T} \int_{\Gamma} c_{\partial \mathfrak{M}}^{\Delta t} \Delta_{\Gamma} \phi(t, x) \mathrm{d} \sigma(x) \mathrm{d} t+\sum_{n=0}^{N-1} \Delta t \sum_{\mathbf{v}=\mathcal{L} \mid \mathcal{L}^{\prime} \in \mathcal{V}}\left(c_{\mathcal{L}}^{n+1}-c_{\mathcal{L}^{\prime}}^{n+1}\right) R_{\mathbf{v}, n+1}^{\phi}
$$

where,

$$
R_{\mathbf{v}, n+1}^{\phi}=\frac{1}{\Delta t} \int_{t^{n}}^{t^{n+1}}\left(\nabla_{\Gamma} \phi(t, \mathbf{v}) n_{\mathbf{v} \mathcal{L}}-\frac{\phi_{\mathcal{L}^{\prime}}^{n+1}-\phi_{\mathcal{L}}^{n+1}}{d_{\mathcal{L}, \mathcal{L}^{\prime}}}\right) \mathrm{d} t .
$$

Using the regularity of the function $\phi$ and bounds on the solutions (4.7) the second term of the right hand side of $T_{5}$ tends to 0 . Then, since $c_{\partial \mathfrak{M}}^{\Delta t}$ converges in $L^{2}\left(0, T ; L^{2}(\Gamma)\right)$ towards $c_{1 \Gamma} \in L^{2}\left(0, T ; H^{1}(\Gamma)\right)$, we finally obtain

$$
T_{5} \underset{\Delta t, \operatorname{size}(\mathcal{T}) \rightarrow 0}{\longrightarrow} \int_{0}^{T} \int_{\Gamma} \nabla_{\Gamma} c_{\text {IГ }}(t, x) \cdot \nabla_{\Gamma} \phi(t, x) \mathrm{d} \sigma(x) \mathrm{d} t .
$$

To finish, as regards the term $T_{6}$, a similar reasoning that for the term $T_{3}$ implies

$$
T_{6} \underset{\Delta t, \operatorname{size}(\mathcal{T}) \rightarrow 0}{\longrightarrow} \int_{0}^{T} \int_{\Gamma} f_{s}^{\prime}\left(c_{i \Gamma}(t, x)\right) \phi(t, x) \mathrm{d} \sigma(x) \mathrm{d} t .
$$

Gathering the relation 4.51) and the convergences 4.53, 4.55, 4.57, 4.58, 4.59) and 4.60 , we conclude the proof.

5. Numerical experiments. In this section, we present some numerical experiments. In order to validate the scheme, we first give numerical error estimates. Then, we present qualitative results to compare the scheme (3.2) with another scheme used in the literature.

In all tests below, in order to ensure orthogonality conditions for the mesh $\mathcal{T}$, we take a Delaunay triangulation of the domain $\Omega$ and for any $\mathcal{K} \in \mathfrak{M}, x_{\mathcal{K}}$ is the circumcenter of $\underline{\mathcal{K}}$ and for any $\mathcal{L} \in \partial \mathfrak{M}, y_{\mathcal{L}}$ is the middle of the chord $\mathbf{e}_{\mathcal{L}}$. We choose the bulk potential equal to the double-well function $f_{b}(c)=c^{2}(1-c)^{2}$ and the corresponding semi-implicit discretization is given by

$$
d^{f_{b}}(x, y)=f_{b}\left(\frac{x+y}{2}\right)-\frac{1}{2}(1-x-y)(x-y)^{2} .
$$

Moreover, let us remark that at each time step, we have to use a Newton method (because of non-linear terms). However, its convergence is achieved in a few inner iterations.

5.1. Numerical error estimates. In this subsection, we compare the discrete solution obtained with the finite-volume scheme with the exact solution. However, there is no explicit non trivial solution, thus we have to modify the Cahn-Hilliard equation (1.7). Indeed, we have to build an exact solution and to add a source term $g_{b}$ in equation 1.1a), another one $g_{s}$ in equation (1.4) and the chemical potential $\mu$ have to satisfy a non homogeneous Neumann boundary condition.

The domain $\Omega$ is the unit circle, thus we can easily obtain the exact coordinates of the centers $x_{\mathcal{L}}$. For a fixed time $t^{n}>0$, for any $\mathcal{K} \in \mathfrak{M}$ (respectively for any $\mathcal{L} \in \partial \mathfrak{M}$ ) we can compare the exact solution $c\left(t^{n}, x_{\mathcal{K}}\right)$ (respectively $c\left(t^{n}, x_{\mathcal{L}}\right)$ ) with the approximate solution $c_{\mathcal{K}}^{n}$ (respectively $\left.c_{\mathcal{L}}^{n}\right)$. We choose the exact solution $c(t,(x, y))=\frac{1}{2}(1+\tanh (5(x+t))$ and we plot, at time $T=N \Delta t=0.5$, the following norms

$$
\frac{\left\|e_{\mathfrak{M}}^{T}\right\|_{L^{2}(\Omega)}}{\left\|c_{\mathfrak{M}}^{T}\right\|_{L^{2}(\Omega)}}, \quad \frac{\left\|e_{\partial \mathfrak{M}}^{T}\right\|_{L^{2}(\Gamma)}}{\left\|c_{\partial \mathfrak{M}}^{T}\right\|_{L^{2}(\Gamma)}}, \quad \frac{\left|e_{\mathcal{T}}^{T}\right|_{1, \mathcal{T}}}{\left|c_{\mathcal{T}}^{T}\right|_{1, \mathcal{T}}} \text { and } \frac{\left|e_{\partial \mathfrak{M}}^{T}\right|_{1, \partial \mathfrak{M}}}{\left|c_{\partial \mathfrak{M}}^{T}\right|_{1, \partial \mathfrak{M}}}
$$


where $e_{\mathcal{T}}^{T}=\left(e_{\mathfrak{M}}^{T}, e_{\partial \mathfrak{M}}^{T}\right)$ and $c_{\mathcal{T}}^{T}=\left(c_{\mathfrak{M}}^{T}, c_{\partial \mathfrak{M}}^{T}\right)$ with

$$
\begin{aligned}
& e_{\mathfrak{M}}^{T}=\left(c\left(T, x_{\mathcal{K}}\right)-c_{\mathcal{K}}^{N}\right)_{\mathcal{K} \in \mathfrak{M}}, \quad e_{\partial \mathfrak{M}}^{T}=\left(c\left(T, x_{\mathcal{L}}\right)-c_{\mathcal{L}}^{N}\right)_{\mathcal{L} \in \partial \mathfrak{M}} \\
& \text { and } \quad c_{\mathfrak{M}}^{T}=\left(c\left(T, x_{\mathcal{K}}\right)\right)_{\mathcal{K} \in \mathfrak{M}}, \quad c_{\partial \mathfrak{M}}^{T}=\left(c\left(T, x_{\mathcal{L}}\right)\right)_{\mathcal{L} \in \partial \mathfrak{M}} \text {. }
\end{aligned}
$$

Then, we plot (see Fig. 5.1 and 5.2 these relative errors

- when the mesh size $\operatorname{size}(\mathcal{T})$ going to 0 and fixed time step;

- when the time step $\Delta t$ tends to 0 and fixed mesh size.

We choose the following parameters: $\varepsilon=0.5, \Gamma_{b}=\sigma_{b}=0.1$ for the bulk and $\Gamma_{s}=10$, $\sigma_{s}=5$ for the surface and $f_{b}(c)=f_{s}(c)=c^{2}(1-c)^{2}$ for the Cahn-Hilliard potentials.

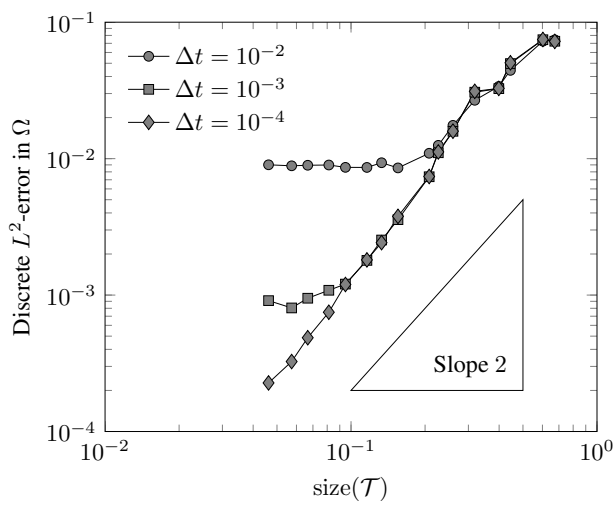

(a) $\frac{\left\|e_{\mathfrak{M}}^{T}\right\|_{L^{2}(\Omega)}}{\left\|c_{\mathfrak{M}}^{T}\right\|_{L^{2}(\Omega)}}$ as a function of $\operatorname{size}(\mathcal{T})$

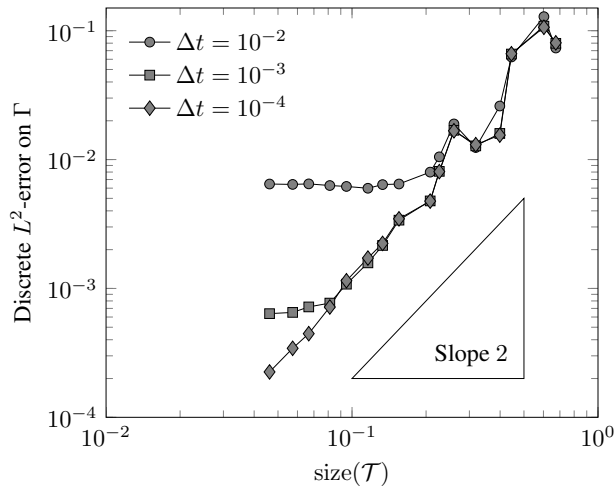

(c) $\frac{\left\|e_{\partial \mathfrak{M}}^{T}\right\|_{L^{2}(\Gamma)}}{\left\|c_{\partial \mathfrak{M}}^{T}\right\|_{L^{2}(\Gamma)}}$ as a function of $\operatorname{size}(\mathcal{T})$

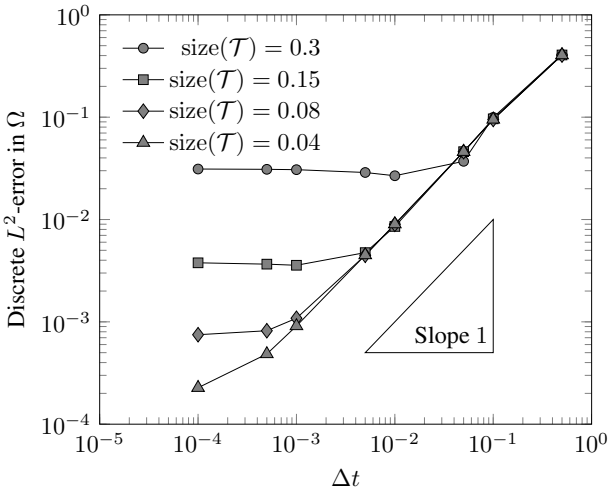

(b) $\frac{\left\|e_{\mathfrak{M}}^{T}\right\|_{L^{2}(\Omega)}}{\left\|c_{\mathfrak{M}}^{T}\right\|_{L^{2}(\Omega)}}$ as a function of $\Delta t$

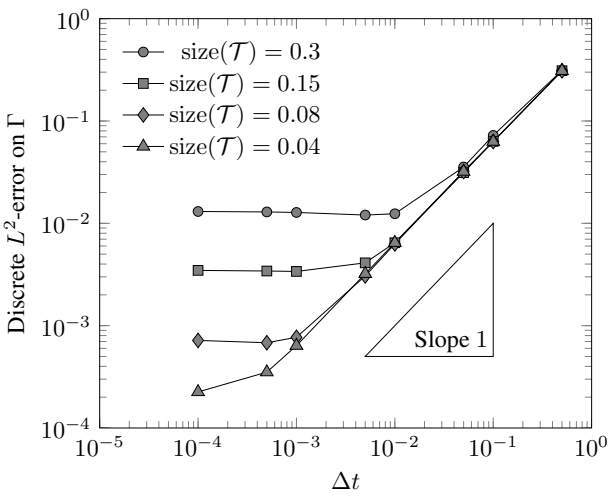

(d) $\frac{\left\|e_{\partial \mathfrak{M}}^{T}\right\|_{L^{2}(\Gamma)}}{\left\|c_{\partial \mathfrak{M}}^{T}\right\|_{L^{2}(\Gamma)}}$ as a function of $\Delta t$

Fig. 5.1: Relative errors in $L^{2}$-norms

As expected, in each case, we observe the first order convergence in time above a certain threshold which depends on the mesh size (see Fig. 5.1b 5.1d 5.2b and 5.2d). As regards the space convergence, above a certain threshold depending on the time step we observe:

- The second order convergence in discrete $L^{2}$-norm both in $\Omega$ and on $\Gamma$ (see Fig. 5.1a et 5.1c. This super-convergence phenomenon is not surprising because it is also 

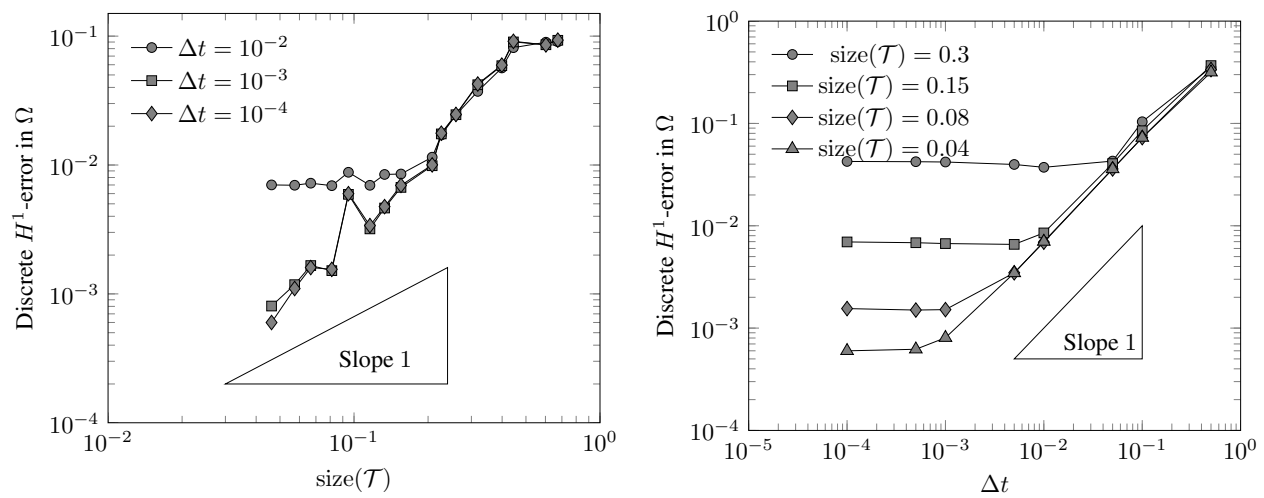

(a) $\frac{\left|e_{\mathcal{T}}^{T}\right|_{1, \mathcal{T}}}{\left|c_{\mathcal{T}}^{T}\right|_{1, \mathcal{T}}}$ as a function of $\operatorname{size}(\mathcal{T})$

(b) $\frac{\left|e_{\mathcal{T}}^{T}\right|_{1, \mathcal{T}}}{\left|c_{\mathcal{T}}^{T}\right|_{1, \mathcal{T}}}$ as a function of $\Delta t$
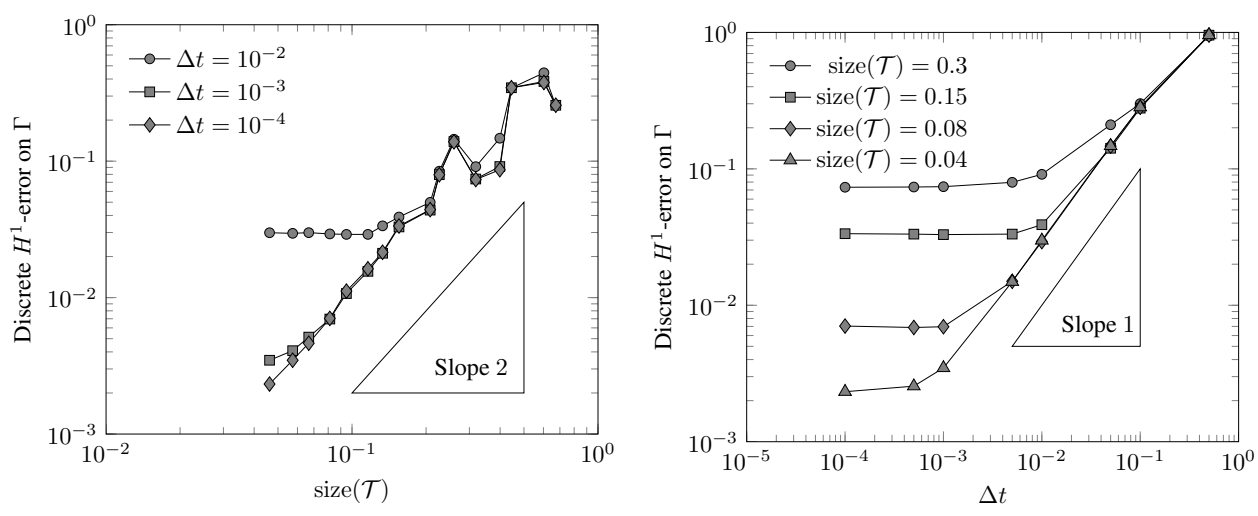

(c) $\frac{\left|e_{\partial \mathfrak{M}}^{T}\right|_{1, \partial \mathfrak{M}}}{\left|c_{\partial \mathfrak{M}}^{T}\right|_{1, \partial \mathfrak{M}}}$ as a function of $\operatorname{size}(\mathcal{T})$

(d) $\frac{\left|e_{\partial \mathfrak{M}}^{T}\right|_{1, \partial \mathfrak{M}}}{\left|c_{\partial \mathfrak{M}}^{T}\right|_{1, \partial \mathfrak{M}}}$ as a function of $\Delta t$

Fig. 5.2: Relative errors in $H^{1}$-discrete seminorms

known for the Laplace problem. From a theoretical point of view this second order convergence remains an open problem.

- When the mesh size is refined, a first order convergence in discrete $H^{1}$-norm in $\Omega$ (see Fig. 5.2a) which is the expected result.

- A second order convergence in discrete $H^{1}$-norm on $\Gamma$ (see Fig. 5.2c). We expect a first order convergence but we observe this super-convergence because of the particular geometry of the boundary mesh.

5.2. Spinodal decomposition. In this subsection, we observe the influence of the dynamic boundary conditions on the phase separation dynamics. The results below are obtained with a smooth curved domain without particular geometry with a mesh size $\operatorname{size}(\mathcal{T}) \sim 0.065$ and a fixed random initial concentration with a fluctuation between 0.49 and 0.51 . Let us remark that for all the test cases below, the initial data is exactly the same.

5.2.1. Influence of the surface diffusion term. We begin by observing the influence of the Laplace-Beltrami operator on the phase separation dynamics. To this end, we choose 
the following fixed parameters: $\Gamma_{b}=0.15, \sigma_{b}=0.006, \varepsilon=0.07$ and $\Gamma_{s}=10$ and we vary the surface capillarity coefficient $\sigma_{s}=0$ or $\sigma_{s}=5$. To compare the results we also consider the case where the order parameter satisfies the homogeneous Neumann boundary condition. Let us notice that in the previous analysis we always assume that $\sigma_{s}>0$ and thus from a theoretical point of view the case $\sigma_{s}=0$ has not been dealt with. We choose a constant time step $\Delta t=10^{-3}$ and we plot:

- The solution at the time $t=0.09$ in $\Omega$ for the three different boundary conditions. The pure phase $c=1$ appears in red, the pure phase $c=0$ in blue and the homogeneous concentration $c=0.5$ is in white.

- The trace of the solution at the same time in function of the curvilinear abscissa by starting from a given point of $\Gamma$ and running along the boundary in the anticlockwise.

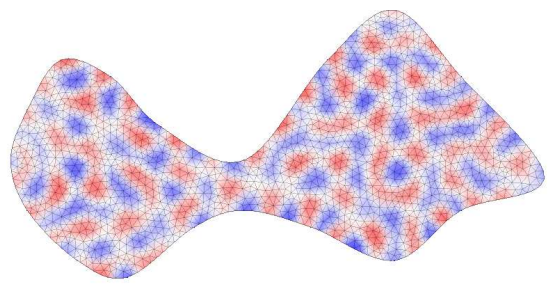

(a) Neumann B.C., Solution in $\Omega$

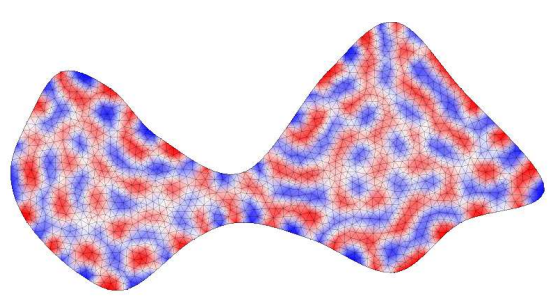

(c) $\sigma_{s}=0$, Solution in $\Omega$

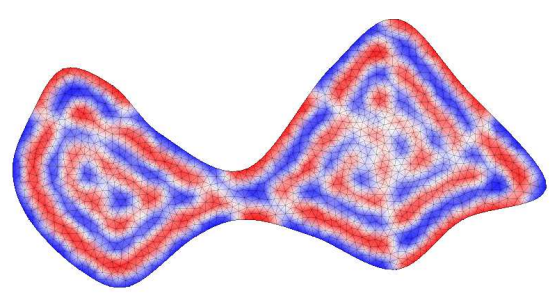

(e) $\sigma_{s}=5$, Solution in $\Omega$

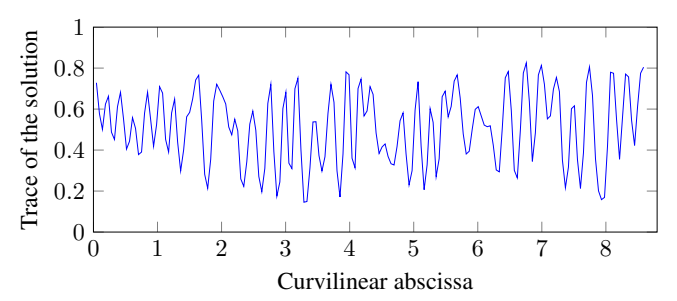

(b) Neumann B.C., Trace of the solution

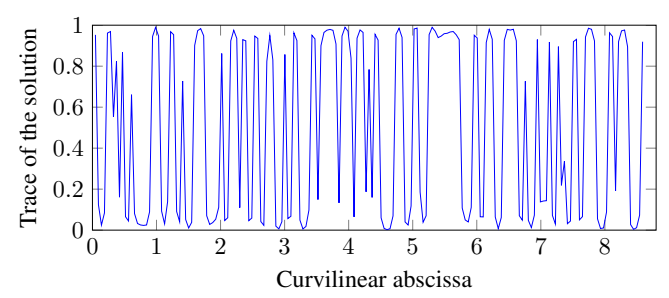

(d) $\sigma_{s}=0$, Trace of the solution

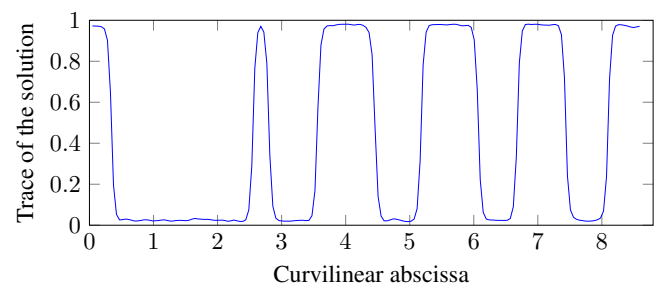

(f) $\sigma_{s}=5$, Trace of the solution

Fig. 5.3: Solution at time $t=0.09$

We observe that several behaviours appear:

- the phase separation is faster with the dynamic boundary conditions than with the Neumann boundary conditions;

- particular structures whose length scale depends on $\sigma_{s}$ : these structure are larger than those which appear in the bulk when $\sigma_{s}>0$ and shorter than those when $\sigma_{s}=0$ (which is in accordance with [12]);

- when $\sigma_{s}=0$ the trace of the solution quickly oscillates between the values 0 and 1 
(approximatively) while for $\sigma_{s}=5$ the value of the trace is much more regular since larger scale structures appear.

- we see emerging patterns which are organized parallel to the boundary.

5.2.2. Preferential attraction by the wall. Here, we consider the case where the physical properties of the wall are such that one of the two components is attracted preferentially by the wall. To model this phenomenon, we consider the surface potential $f_{s}(c)=\frac{g_{s}}{2} c^{2}-\frac{h_{s}+g_{s}}{2} c$ with $g_{s}>0$, thus

- when $h_{s}=0$ the minimum of $f_{s}$ is in $c=\frac{1}{2}$ thus the wall has the same behaviour with respect to the two components;

- when $h_{s}>0$ the minimum of $f_{s}$ is in $c=\frac{h_{s}+g_{s}}{2 g_{s}}>\frac{1}{2}$ thus the wall attracts preferentially the phase $c=1$;

- conversely when $h_{s}<0$ the minimum of $f_{s}$ is in $c=\frac{h_{s}+g_{s}}{2 g_{s}}<\frac{1}{2}$ thus the wall attracts preferentially the phase $c=0$.

Let us notice that the semi-implicit discretization of this surface potential is written

$$
d^{f_{s}}(x, y)=f_{s}^{\prime}\left(\frac{x+y}{2}\right) .
$$

We choose the following fixed parameters: $\Gamma_{b}=0.3, \sigma_{b}=0.008, \varepsilon=0.1, \sigma_{s}=0.1$, $\Gamma_{s}=10$ and $g_{s}=1$ and we vary the coefficient $h_{s}$. These results are given at time $t=0.25$ with a time step $\Delta t=10^{-3}$.

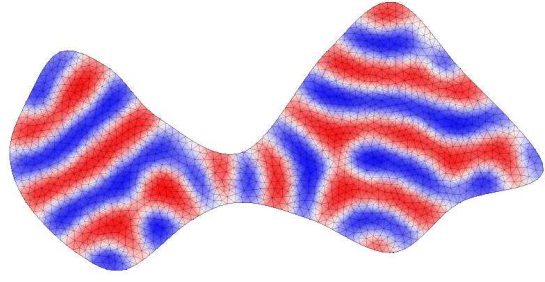

(a) $h_{s}=0$

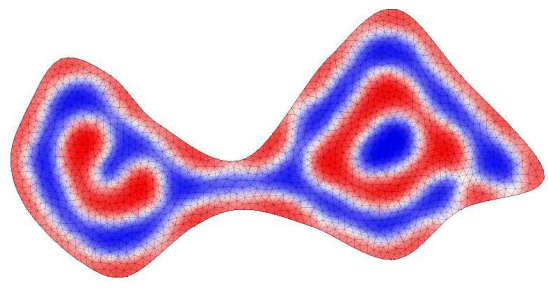

(c) $h_{s}=0.2$

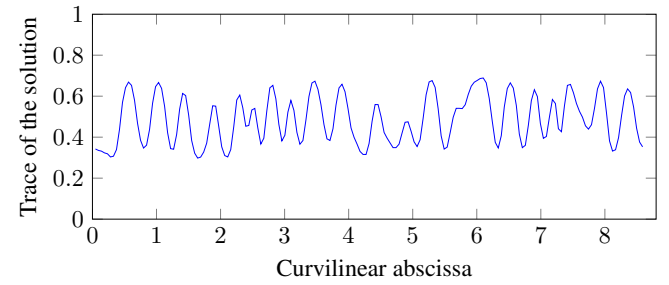

(b) $h_{s}=0$

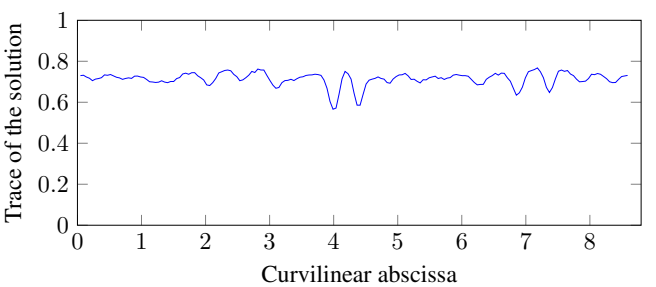

(d) $h_{s}=0.2$

Fig. 5.4: Influence of the parameter $h_{s}$

The parallel structures that we observe when $h_{s}=0$ (see Fig 5.4a) are similar to those observed in [6, 16] and the alternation between the phases confirms that the wall does not prefer one of the two components. On the other hand, in Fig. 5.4c the wall exerts a preferential attraction on the phase $c=1$ (in red) that is in accordance with the choice of $h_{s}>0$.

6. Conclusion. We proposed here a finite-volume scheme for the Cahn-Hilliard equation with dynamic boundary conditions in a smooth domain. 
At the theoretical level, we are able to show the convergence of the discrete solution towards a weak solution of the continuous problem (and thus, in particular, the existence of this solution). For this purpose, we give a new space translation estimates which gives a limit in $L^{\infty}\left(0, T, H^{1}(\Omega)\right)$ whose trace is in $L^{\infty}\left(0, T, H^{1}(\Gamma)\right)$.

At the numerical level, the method allows an easy implementation for the coupling between the equation in the domain and whose on its boundary even with a curved geometry for the domain. We have also presented numerical error estimates which allow us to validate the scheme and simulations which illustrate the different properties of the Cahn-Hilliard model with dynamic boundary conditions depending on physical properties of the system.

In [5] we give a more complex method called DDFV method to solve this problem which enables us to use more general meshes without orthogonality condition as for example non-conforming mesh. We also propose an original DDFV scheme to study the CahnHilliard/Stokes phase field model.

\section{REFERENCES}

[1] B. Andreianov, F. Boyer, and F. Hubert. Discrete duality finite volume schemes for Leray-Lions-type elliptic problems on general 2D meshes. Numer. Methods Partial Differential Equations, 23(1):145-195, 2007.

[2] M. Bessemoulin-Chatard, C. Chainais-Hillairet, and F. Filbet. On discrete functional inequalities for some finite volume schemes. to appear, http://hal.archives-ouvertes.fr/hal-00672591

[3] P. Bousquet, F. Boyer, and F. Nabet. On a functional inequality arising in the analysis of finite volume methods. in preparation, 2014.

[4] F. Boyer and S. Minjeaud. Numerical schemes for a three component Cahn-Hilliard model. ESAIM Math. Model. Numer. Anal., 45(4):697-738, 2011.

[5] F. Boyer and F. Nabet. A DDFV method for a Cahn-Hilliard-Stokes phase field model. in preparation, 2014

[6] L. Cherfils, M. Petcu, and M. Pierre. A numerical analysis of the Cahn-Hilliard equation with dynamic boundary conditions. Discrete Contin. Dyn. Syst., 27(4):1511-1533, 2010.

[7] R. Chill, E. Fašangová, and J. Prüss. Convergence to steady state of solutions of the Cahn-Hilliard and Caginalp equations with dynamic boundary conditions. Math. Nachr., 279(13-14):1448-1462, 2006.

[8] K. Deimling. Nonlinear functional analysis. Springer-Verlag, Berlin, 1985.

[9] J. Droniou. A density result in Sobolev spaces. J. Math. Pures Appl. (9), 81(7):697-714, 2002.

[10] R. Eymard, T. Gallouët, and R. Herbin. Finite volume methods. Handbook of Numerical Analysis, 7:713$1018,2000$.

[11] R. Eymard and T. Gallouët. H-convergence and numerical schemes for elliptic problems. SIAM J. OF NUM. ANAL, pages 539-562, 2002.

[12] H.P. Fischer, P. Maass, and W. Dieterich. Novel surface modes in spinodal decomposition. Phys. Rev. Lett., 79:893-896, Aug 1997.

[13] H.P. Fischer, P. Maass, and W. Dieterich. Diverging time and length scales of spinodal decomposition modes in thin films. EPL (Europhysics Letters), 42(1):49-54, 1998.

[14] T. Gallouët, R. Herbin, A. Larcher, and J.-C. Latché. Analysis of a fractional-step scheme for the p1 radiative diffusion model. to appear, http://hal.archives-ouvertes.fr/hal-00477086

[15] G. Gilardi, A. Miranville, and G. Schimperna. On the Cahn-Hilliard equation with irregular potentials and dynamic boundary conditions. Commun. Pure Appl. Anal., 8(3):881-912, 2009.

[16] R. Kenzler, F. Eurich, P. Maass, B. Rinn, J. Schropp, E. Bohl, and W. Dieterich. Phase separation in confined geometries: Solving the Cahn-Hilliard equation with generic boundary conditions. j-COMP-PHYS-COMM, 133:139-157, Jan 2001.

[17] A. Miranville and S. Zelik. Exponential attractors for the Cahn-Hilliard equation with dynamic boundary conditions. Math. Methods Appl. Sci., 28(6):709-735, 2005.

[18] J. Prüss, R. Racke, and S. Zheng. Maximal regularity and asymptotic behavior of solutions for the CahnHilliard equation with dynamic boundary conditions. Ann. Mat. Pura Appl. (4), 185(4):627-648, 2006.

[19] R. Racke and S. Zheng. The Cahn-Hilliard equation with dynamic boundary conditions. Adv. Differential Equations, 8(1):83-110, 2003.

[20] $\mathrm{H}$. Wu and S. Zheng. Convergence to equilibrium for the Cahn-Hilliard equation with dynamic boundary conditions. J. Differential Equations, 204(2):511-531, 2004. 